\title{
Supplementary Material File ESI1
}

\section{DETAILED EXPERIMENTAL \\ Chemical and biochemical reactivity of the reduced forms of nicotinamide riboside}

\section{Mikhail V. Makarov*, Faisal Hayat", Briley Graves, Manoj Sonavane, Edward A. Salter, Andrzej Wierzbicki, Natalie R. Gassman and Marie E. Migaud ${ }^{\$}$}

${ }^{\$}$ Corresponding author: mmigaud@southalabama.edu

*Joint first authorship.

Table of contents

\begin{tabular}{|c|c|}
\hline & Page \\
\hline EXPERIMENTAL & 1 \\
\hline General remarks & 1 \\
\hline Scheme 1S. Synthesis of 1,2-NRH, 1,4-NRH and 1,6-NRH & 2 \\
\hline$N$-(trimethylsilyl)nicotinamide & 2 \\
\hline $\begin{array}{l}\text { 3-Carbamoyl-1-((2R,3R,4R,5R)-3,4-diacetoxy-5-(acetoxymethyl)-tetrahydrofuran-2- } \\
\text { yl)pyridin-1-ium trifluoromethanesulfonate }(\mathbf{6})\end{array}$ & 3 \\
\hline $\begin{array}{l}(2 R, 3 R, 4 R, 5 R)-2 \text {-(Acetoxymethyl)-5-(3-carbamoylpyridin-1(4H)-yl)tetrahydrofuran-3,4- } \\
\text { diyl diacetate (7) }\end{array}$ & 3 \\
\hline $\begin{array}{l}\text { 1-((2R,3R,4S,5R)-3,4-Dihydroxy-5-(hydroxymethyl)tetrahydrofuran-2-yl)-1,4- } \\
\text { dihydropyridine-3-carboxamide (1) }\end{array}$ & 3 \\
\hline $\begin{array}{l}\text { Reduction of NRTA OTf with sodium borohydride in DCM/water: Synthesis of 1,2- } \\
\text { NRH TA (5) and 1,6-NRH TA (9) }\end{array}$ & 4 \\
\hline $\begin{array}{l}\text { Reduction of NRTA OTf with sodium borohydride in DMF: Synthesis of compounds } 8 \\
(1,2-\mathrm{NRH} \text { TA) and } \mathbf{9}(1,6-\mathrm{NRH} \text { TA) }\end{array}$ & 4 \\
\hline $\begin{array}{l}(2 R, 3 R, 4 R, 5 R) \text {-2-(acetoxymethyl)-5-(3-carbamoylpyridin-1(2H)-yl)tetrahydrofuran-3,4- } \\
\text { diyl diacetate }(\mathbf{8}, 1,2 \text {-NRH TA) }\end{array}$ & 5 \\
\hline $\begin{array}{l}(2 R, 3 R, 4 R, 5 R)-2 \text {-(acetoxymethyl)-5-(5-carbamoylpyridin-1(2H)-yl)tetrahydrofuran-3,4- } \\
\text { diyl diacetate }(9,1,6 \text {-NRH TA) }\end{array}$ & 5 \\
\hline General procedure for the synthesis of 1,2-NRH (2) and 1,6-NRH (3) & 6 \\
\hline $\begin{array}{l}\text { 1-((2R,3R,4S,5R)-3,4-dihydroxy-5-(hydroxymethyl)tetrahydrofuran-2-yl)-1,2- } \\
\text { dihydropyridine-3-carboxamide }(2,1,2-\mathrm{NRH})\end{array}$ & 6 \\
\hline $\begin{array}{l}\text { 1-((2R,3R,4S,5R)-3,4-dihydroxy-5-(hydroxymethyl)tetrahydrofuran-2-yl)-1,6- } \\
\text { dihydropyridine-3-carboxamide }(3,1,6-\mathrm{NRH})\end{array}$ & 6 \\
\hline Table S1. ${ }^{1} \mathrm{H}$ NMR data of compounds $7, \mathbf{8}, \mathbf{9}$ and $\mathbf{1}, \mathbf{2}, \mathbf{3}$. & 7 \\
\hline Table S2. ${ }^{3} \mathrm{C}$ NMR data of compounds $\mathbf{7 , 8 , 9}$ and $\mathbf{1}, \mathbf{2}, \mathbf{3}$. & 8 \\
\hline Hydration of NRH Using Buffer & 9 \\
\hline Figure S1 NMR-ESI Isomerism of NRH and NR+: & 9 \\
\hline $\begin{array}{l}\text { Figure S2 Buffer conditions affect the stability of 1,2-, 1,4- and 1,6-NRH in solution in } \\
\text { absence of NQO2 }\end{array}$ & 10 \\
\hline $\begin{array}{l}\text { Figure S3 Buffer conditions affect the product distribution of 1,6-NRH in solution in } \\
\text { absence of NQO2 }\end{array}$ & 10 \\
\hline $\begin{array}{l}\text { Figure S4 Changes in the product distribution of 1, 2-, 1,4- and 1,6-NRH in solution in } \\
\text { absence and presence of NOO2 measured by }{ }^{1} \mathrm{H} \text { NMR }\end{array}$ & 11 \\
\hline $\begin{array}{l}\text { Scheme S1: Stability and reactivity of the three dihydronicotinamide ribosides under } \\
\text { NQO2 enzymatic conditions. }\end{array}$ & 11 \\
\hline Cytotoxicity studies & 12 \\
\hline Recombinant human NQO2 enzyme activity. & 12 \\
\hline Identification of Modified Lysine Residues in BSA & 13 \\
\hline Quantum-based Computational Modeling NQO2. & 16 \\
\hline $\begin{array}{l}\text { Figure S5: Superposition of 1,4- and 1,6-NRH bound to the face of the flavin moiety of } \\
\text { FAD in FAD-bound NQO2. }\end{array}$ & 17 \\
\hline
\end{tabular}


General remarks. NMR spectra were recorded on a Bruker Avance III HD 400 spectrometer $\left({ }^{1} \mathrm{H}\right.$, $400.11 ;{ }^{19} \mathrm{~F}, 376.44$; and $\left.{ }^{13} \mathrm{C}, 100.62 \mathrm{MHz}\right)$ using residual proton signal $\left({ }^{1} \mathrm{H}\right)$ and that of carbon atom $\left({ }^{13} \mathrm{C}\right)$ of a deuterated solvent as an internal standard relative to TMS, and $\mathrm{CFCl}_{3}\left({ }^{19} \mathrm{~F}\right)$ as an external standard. Column chromatography was performed on silica gel columns using Teledyne medium pressure liquid chromatography system with UV monitoring of eluted fractions (at $280 \mathrm{~nm}$ and $350 \mathrm{~nm}$ ). Analytical TLCs were performed with Merck silica gel 60 F254 plates; visualization of TLCs was accomplished by UV light. HRMS spectra were obtained on LTQ Orbitrap XL Mass Spectrometer (HESI source, positive polarity, capillary temp $200^{\circ} \mathrm{C}$, source voltage $3.0 \mathrm{kV}$ ).

All commercial reagents were purchased from VWR and used without further purification. Anhydrous DCM was obtained by distillation of commercial DCM (from VWR) over calcium hydride. Anhydrous commercial DMF was kept over calcinated molecular sieves. Anhydrous $\mathrm{MeOH}$ was prepared by distillation over $\mathrm{Mg}$ in the presence of iodine according to standard procedure and kept afterward over molecular sieves ( $c f:$ W.L.F. Armarego and C.L.L. Chai, Purification of Laboratory Chemicals, $6^{\text {th }}$ Ed., Butterworth-Heinemann/Elsevier, Burlington/Oxford, 2009).

Reduction of pyridinium salts, including $N$-alkylated nicotinamide salts, to mixtures of corresponding isomeric 1,2-, 1,4-, and 1,6-dihydropyridines, in anhydrous DMF at $0^{\circ} \mathrm{C}$ along with the assignment of proton signals for the 1,2-, 1,4-, and 1,6-dihydropyridine core in the ${ }^{1} \mathrm{H}$ NMR spectra was previously described in the literature and relied upon in this work ( $c f:$ R.B. Schmidt, G. Berger, Chem. Ber. 1976, 109, 2936-2947).

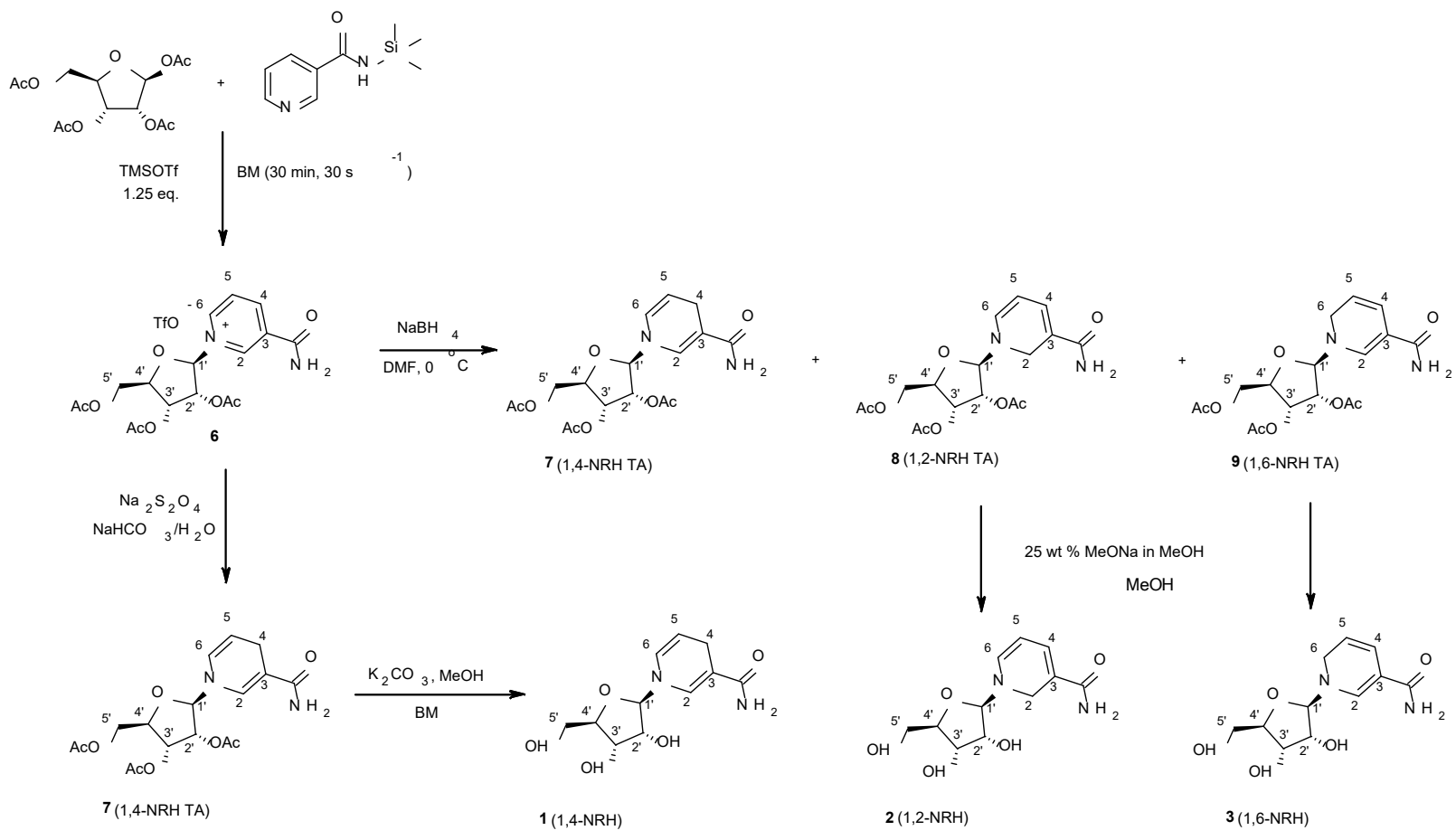

Scheme S1. Synthesis of 1,2-NRH, 1,4-NRH and 1,6-NRH. 
$N$-(Trimethylsilyl)nicotinamide. A $250 \mathrm{~mL}$ round-bottom two-neck flask equipped with a reflux condenser was charged with nicotinamide $(5.00 \mathrm{~g} ; 0.041 \mathrm{~mol})$ followed by the<smiles>C[Si](C)(C)NC(=O)c1cccnc1</smiles>
addition of HMDS (45 mL; $34.6 \mathrm{~g} ; 0.215 \mathrm{~mol} ; 5.2$ equiv.) and TMSCl (10.4 mL; $8.9 \mathrm{~g} ; 0.082 \mathrm{~mol} ; 2$ equiv.) via a syringe in one portion. The reaction mixture was heated in an oil bath to $105^{\circ} \mathrm{C}$ (oil bath temperature) at stirring and was left under these conditions overnight. The next day, a clear, colorless solution formed; the flask was taken from the heating oil bath and the warm reaction solution was transferred into a roundbottom single-neck flask through a cannula. The solution was evaporated to dryness on a rotary evaporator followed by drying under high vacuum to give colorless crystalline product, $7.95 \mathrm{~g}(100 \%) .{ }^{1} \mathrm{H}$ NMR $\left(\mathrm{C}_{6} \mathrm{D}_{6}\right), \delta$, ppm: 0.00 (br s, 9H, $\mathrm{SiMe}_{3}$ ), 5.89 (br s, $\left.1 \mathrm{H}, \mathrm{NH}\right), 6.38$ (dd, $\left.1 \mathrm{H},{ }^{3} \mathrm{~J}_{\mathrm{HH}}=4.9 \mathrm{~Hz},{ }^{3} \mathrm{~J}_{\mathrm{HH}}=8.0 \mathrm{~Hz}, \mathrm{H} 5\right)$, $7.62\left(\mathrm{~d}, 1 \mathrm{H},{ }^{3} \mathrm{~J}_{\mathrm{HH}}=8.0 \mathrm{~Hz}, \mathrm{H} 4\right), 8.12\left(\mathrm{~d}, 1 \mathrm{H},{ }^{3} \mathrm{~J}_{\mathrm{HH}}=4.7 \mathrm{~Hz}, \mathrm{H} 6\right), 8.82(\mathrm{br} \mathrm{s}, 1 \mathrm{H}, \mathrm{H} 2) .{ }^{13} \mathrm{C} \mathrm{NMR}\left(\mathrm{CDCl}_{3}\right), \delta$, ppm: 0.22 ( $\mathrm{SiMe}_{3}$ ), 124.10 (C5), 131.81 (C3), 136.22 (C4), 149.88 (C2), 153.07 (C6), 171.35 (CO).

\section{3-Carbamoyl-1-((2R,3R,4R,5R)-3,4-diacetoxy-5-(acetoxymethyl)tetrahydrofuran-2-}

yl)pyridin-1-ium trifluoromethanesulfonate (6). A PTFE jar was charged with $N$ -

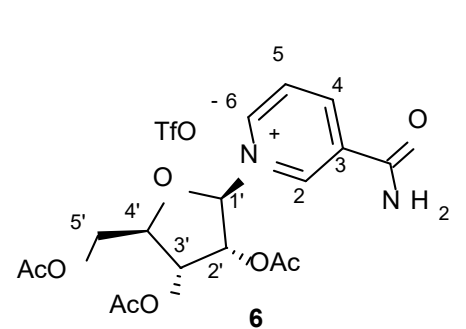
(trimethylsilyl)nicotinamide $(0.80 \mathrm{~g} ; 0.004 \mathrm{~mol}), 1,2,3,5$-tetra- $O$-acetylD- $\beta$-ribofuranose $(1.31 \mathrm{~g} ; 0.004 \mathrm{~mol})$ and anhydrous DCM $(0.4 \mathrm{~mL} ; 0.006$ mol) was added, followed by addition of TMSOTf (1.13 g; $0.005 \mathrm{~mol})$. The reagents were subjected to ball-milling on a Retsch MM400 miller for $30 \mathrm{~min}$ at $30 \mathrm{~Hz}$. The jar was allowed to cool down to room temperature. The content of the jar was dissolved in DCM $(2 \times 10 \mathrm{~mL})$ and the yellow solution was transferred into a round bottom flask. Volatiles were removed on a rotary evaporator to dryness to give a yellow foam $(2.92 \mathrm{~g}$; ca. quantitative yield as calculated on $\mathrm{N}$-silylated form of 6 ) triturated by a spatula resulting in a yellow powder. The product containing residues of acetic acid and silylated residues was used on the next step without additional purification. ${ }^{19} \mathrm{~F}$ NMR $\left(\mathrm{D}_{2} \mathrm{O}\right), \delta$, ppm: -78.83. ${ }^{1} \mathrm{H}$ NMR $\left(\mathrm{D}_{2} \mathrm{O}\right), \delta$, ppm: 1.95 (Me from acetic acid residue), 2.02 (s, 3H, Me), 2.06 (s, 3H, Me), 2.09 (s, 3H, Me), 4.42-4.50 (m, 2H, H5'), 4.80-4.83 (m, 1H, $\mathrm{H}^{\prime}$ ), 5.38 (apparent t, $\left.1 \mathrm{H},{ }^{3} \mathrm{~J}_{\mathrm{HH}}=5.4 \mathrm{~Hz}, \mathrm{H} 3^{\prime}\right), 5.49$ (dd, $\left.1 \mathrm{H},{ }^{3} \mathrm{~J}_{\mathrm{HH}}=3.8 \mathrm{~Hz},{ }^{3} \mathrm{~J}_{\mathrm{HH}}=5.8 \mathrm{~Hz}, \mathrm{H} 2^{\prime}\right), 6.51(\mathrm{~d}, 1 \mathrm{H}$, $\left.{ }^{3} \mathrm{~J}_{\mathrm{HH}}=3.7 \mathrm{~Hz}, \mathrm{H} 1^{\prime}\right), 8.21\left(\mathrm{dd}, 1 \mathrm{H},{ }^{3} \mathrm{~J}_{\mathrm{HH}}=6.5 \mathrm{~Hz},{ }^{3} \mathrm{~J}_{\mathrm{HH}}=8.7 \mathrm{~Hz}, \mathrm{H} 5\right), 8.92\left(\mathrm{~d}, 1 \mathrm{H},{ }^{3} \mathrm{~J}_{\mathrm{HH}}=8.0 \mathrm{~Hz}, \mathrm{H} 4\right), 9.13(\mathrm{~d}$, $\left.1 \mathrm{H},{ }^{3} \mathrm{~J}_{\mathrm{HH}}=6.4 \mathrm{~Hz}, \mathrm{H} 6\right), 9.37$ (s, 1H, H2). ${ }^{13} \mathrm{C} \mathrm{NMR}\left(\mathrm{D}_{2} \mathrm{O}\right), \delta$, ppm: $19.92(\mathrm{Me}), 19.97(\mathrm{Me}), 20.31(\mathrm{Me})$, 20.52 (Me from acetic acid residue), $62.77\left(\mathrm{C5}^{\prime}\right), 69.54\left(\mathrm{C}^{\prime}\right), 76.50\left(\mathrm{C} 2^{\prime}\right), 82.78$ (C4'), $97.48\left(\mathrm{C1}^{\prime}\right), 119.77$ $\left(\mathrm{CF}_{3},{ }^{1} \mathrm{~J}_{\mathrm{CF}}=316 \mathrm{~Hz}\right), 128.78$ (C5), $134.36(\mathrm{C} 3), 140.58(\mathrm{C} 2), 143.23$ (C6), $146.39(\mathrm{C} 4), 165.60\left(\mathrm{CONH}_{2}\right)$, $172.53(\mathrm{CO}), 172.58(\mathrm{CO}), 173.48(\mathrm{CO}), 176.77$ (CO from acetic acid residue). MS: found $\mathrm{m} / \mathrm{z}=380.84$ (M). HRMS found: 381.12965. Calculated for $\mathrm{C}_{17} \mathrm{H}_{21} \mathrm{~N}_{2} \mathrm{O}_{8}(\mathrm{M}): 381.12924$.

$(2 R, 3 R, 4 R, 5 R)-2$-(Acetoxymethyl)-5-(3-carbamoylpyridin-1(4H)-l)tetrahydrofuran-3,4-diyl diacetate (7). In a round bottom flask flushed with nitrogen, compound 6 ( $2.85 \mathrm{~g}$, ca. $0.004 \mathrm{~mol})$ was

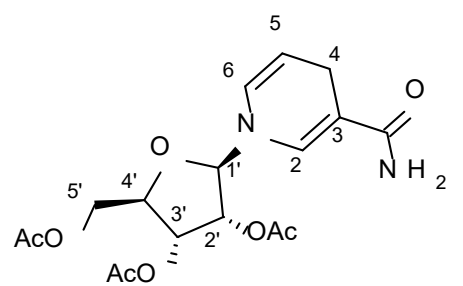
dissolved in a nitrogen-purged DCM $(40 \mathrm{~mL})$ and $13 \mathrm{~mL}$ of saturated aqueous $\mathrm{NaHCO}_{3}$ solution were added, followed by addition of solid sodium dithionite (ca. $85 \% ; 4.27 \mathrm{~g} ; 0.021 \mathrm{~mol}$ ) and $7 \mathrm{~mL}$ of water at stirring and at room temperature. The biphasic reaction mixture was stirred at room temperature for $4 \mathrm{~h}$, and then brine $(30 \mathrm{~mL})$ and DCM $(40 \mathrm{~mL})$ were added. Yellow organic phase was separated, washed twice with brine, dried over $\mathrm{Na}_{2} \mathrm{SO}_{4}$, filtered and evaporated under reduced pressure to give light yellow foam (1.35 g; 84\% based on compound 3). ${ }^{1} \mathrm{H}$ NMR $\left(\mathrm{CDCl}_{3}\right), \delta$, ppm: 2.02 (s, 3H, Me), $2.04(\mathrm{~s}, 3 \mathrm{H}, \mathrm{Me})$, $2.10(\mathrm{~s}, 3 \mathrm{H}, \mathrm{Me}), 3.06$ (q, $\left.2 \mathrm{H},{ }^{3} \mathrm{~J}_{\mathrm{HH}}=1.4 \mathrm{~Hz}, \mathrm{H} 4\right), 4.11\left(\mathrm{dd}, 1 \mathrm{H},{ }^{3} \mathrm{~J}_{\mathrm{HH}}=4.7 \mathrm{~Hz},{ }^{3} \mathrm{~J}_{\mathrm{HH}}=3.2 \mathrm{~Hz}, \mathrm{H} 4^{\prime}\right), 4.20-4.21$ $\left(\mathrm{m}, 2 \mathrm{H}, \mathrm{H} 5^{\prime}\right), 4.80\left(\mathrm{dt}, 1 \mathrm{H},{ }^{3} \mathrm{~J}_{\mathrm{HH}}=3.4 \mathrm{~Hz},{ }^{3} \mathrm{~J}_{\mathrm{HH}}=8.2 \mathrm{~Hz}, \mathrm{H} 5\right), 4.89\left(\mathrm{~d}, 1 \mathrm{H},{ }^{3} \mathrm{~J}_{\mathrm{HH}}=7.0 \mathrm{~Hz}, \mathrm{H} 1^{\prime}\right), 5.11(\mathrm{t}, 1 \mathrm{H}$, 
$\left.{ }^{3} \mathrm{~J}_{\mathrm{HH}}=6.4 \mathrm{~Hz}, \mathrm{H} 2^{\prime}\right), 5.18\left(\mathrm{dd}, 1 \mathrm{H},{ }^{3} \mathrm{~J}_{\mathrm{HH}}=2.8 \mathrm{~Hz},{ }^{3} \mathrm{~J}_{\mathrm{HH}}=5.8 \mathrm{~Hz}, \mathrm{H3}{ }^{\prime}\right), 5.27\left(\mathrm{br} \mathrm{s}, 2 \mathrm{H}, \mathrm{NH}_{2}\right), 5.88\left(\mathrm{dd}, 1 \mathrm{H},{ }^{4} \mathrm{~J}_{\mathrm{HH}}=\right.$ $\left.1.7 \mathrm{~Hz},{ }^{3} \mathrm{~J}_{\mathrm{HH}}=8.2 \mathrm{~Hz}, \mathrm{H} 6\right), 7.09$ (s, 1H, H2). ${ }^{13} \mathrm{C} \mathrm{NMR}\left(\mathrm{CDCl}_{3}\right), \delta$, ppm: 18.47 (Me), 18.59 (Me), 18.79 (Me), 21.22 (C4), $61.60\left(\mathrm{C5}^{\prime}\right), 68.87$ and 68.91 (C3' and $\left.\mathrm{C2} 2^{\prime}\right), 77.05$ (C4'), $91.36\left(\mathrm{Cl}^{\prime}\right), 100.38$ (C3), 102.49 (C5), 123.07 (C6), 134.36 (C2), 167.52 (two overlapped CO), 167.53 (CO), 168.55 (CO). MS: found m/z $=382.96(\mathrm{M}+1)$. HRMS found: 383.14530 . Calculated for $\mathrm{C}_{17} \mathrm{H}_{23} \mathrm{~N}_{2} \mathrm{O}_{8}(\mathrm{M}+1)$ : 383.14489 .

1-((2R,3R,4S,5R)-3,4-Dihydroxy-5-(hydroxymethyl)tetrahydrofuran-2-yl)-1,4-

dihydropyridine-3-carboxamide (1). A PTFE jar was charged with compound 7 (0.50 g; $0.0013 \mathrm{~mol})$, anhydrous potassium carbonate $(0.0180 \mathrm{~g} ; 0.00013 \mathrm{~mol})$ and methanol $(0.3 \mathrm{~mL} ; 0.238 \mathrm{~g} ; 0.0074 \mathrm{~mol})$ were

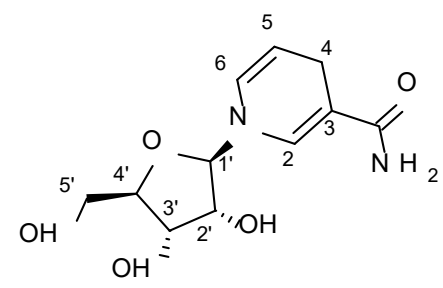
added. The reagents were subjected to ball-milling on a Retsch MM400 miller for $25 \mathrm{~min}$ at $25 \mathrm{~Hz}$. The jar was allowed to cool down to room temperature. The content of the jar was dissolved in methanol $(2 \times 10 \mathrm{~mL})$ and the yellow solution was transferred into a round bottom flask. Volatiles were removed on a rotary evaporator to dryness to give a yellow foam that was triturated with diethyl ether resulting in a yellow powder. Diethyl ether was removed by decantation and the product was dried under reduced pressure at $34^{\circ} \mathrm{C}$. Yield: $0.34 \mathrm{~g}$ (ca. $\left.100 \%\right) .{ }^{1} \mathrm{H}$ NMR $\left(\mathrm{D}_{2} \mathrm{O}\right), \delta$, ppm: 2.98 (q, $\left.2 \mathrm{H},{ }^{3} \mathrm{~J}_{\mathrm{HH}}=1.5 \mathrm{~Hz}, \mathrm{H} 4\right), 3.26(\mathrm{~s}, 1.5 \mathrm{H}, 0.5 \mathrm{MeOH}), 3.60$ and 3.66 (AB part of ABX system, $2 \mathrm{H}$, $\mathrm{J}_{\mathrm{AB}}=12.5 \mathrm{~Hz}, \mathrm{~J}_{\mathrm{BX}}=4.8 \mathrm{~Hz}, \mathrm{~J}_{\mathrm{AX}}=3.6 \mathrm{~Hz}, \mathrm{H}^{\prime}{ }_{\mathrm{A}}$ and $\left.\mathrm{H} 5^{\prime}{ }_{\mathrm{B}}\right), 3.88\left(\mathrm{dd}, 1 \mathrm{H},{ }^{3} \mathrm{~J}_{\mathrm{HH}}=6.8 \mathrm{~Hz},{ }^{3} \mathrm{~J}_{\mathrm{HH}}=3.5 \mathrm{~Hz}, \mathrm{H} 4^{\prime}\right)$, $4.04\left(\mathrm{dd}, 1 \mathrm{H},{ }^{3} \mathrm{~J}_{\mathrm{HH}}=2.9 \mathrm{~Hz},{ }^{3} \mathrm{~J}_{\mathrm{HH}}=5.6 \mathrm{~Hz}, \mathrm{H} 3^{\prime}\right), 4.11\left(\mathrm{t}, 1 \mathrm{H},{ }^{3} \mathrm{~J}_{\mathrm{HH}}=6.3 \mathrm{~Hz}, \mathrm{H} 2^{\prime}\right), 4.66\left(\mathrm{OH}, \mathrm{NH}_{2}\right.$ overlapped with $\left.\mathrm{D}_{2} \mathrm{O}\right), 4.79\left(\mathrm{~d}, 1 \mathrm{H},{ }^{3} \mathrm{~J}_{\mathrm{HH}}=7.0 \mathrm{~Hz}, \mathrm{H1}\right.$ ) $, 4.90\left(\mathrm{dt}, 1 \mathrm{H},{ }^{3} \mathrm{~J}_{\mathrm{HH}}=3.4 \mathrm{~Hz},{ }^{3} \mathrm{~J}_{\mathrm{HH}}=8.2 \mathrm{~Hz}, \mathrm{H} 5\right), 6.01(\mathrm{dd}, 1 \mathrm{H}$, $\left.{ }^{4} \mathrm{~J}_{\mathrm{HH}}=1.5 \mathrm{~Hz},{ }^{3} \mathrm{~J}_{\mathrm{HH}}=8.2 \mathrm{~Hz}, \mathrm{H} 6\right), 7.06(\mathrm{~s}, 1 \mathrm{H}, \mathrm{H} 2) .{ }^{13} \mathrm{C} \mathrm{NMR}\left(\mathrm{D}_{2} \mathrm{O}\right), \delta, \mathrm{ppm}: 22.07(\mathrm{C} 4), 49.00(\mathrm{MeOH})$, 61.63 (C5'), $70.21\left(\mathrm{C}^{\prime}\right), 71.11\left(\mathrm{C} 2^{\prime}\right), 83.56\left(\mathrm{C}^{\prime}\right), 95.03$ (C1'), 101.08 (C3), 105.20 (C5), 125.37 (C6), $137.80(\mathrm{C} 2), 172.96(\mathrm{CO})$. MS: found $\mathrm{m} / \mathrm{z}=257.21(\mathrm{M}+1)$. HRMS found: 257.11376 . Calculated for $\mathrm{C}_{11} \mathrm{H}_{17} \mathrm{~N}_{2} \mathrm{O}_{5}(\mathrm{M}+1): 257.11320$

Reduction of NRTA OTf (6) with sodium borohydride in DCM/water: Synthesis of 1,2-NRH TA (8) and 1,6-NRH TA (9). Compound 6 (4.25 g; prepared from $1.43 \mathrm{~g}(0.0074 \mathrm{~mol})$ of silylated nicotinamide and $2.35 \mathrm{~g}(0.0074 \mathrm{~mol})$ of 1,2,3,5-tetra- $O$-acetyl-D- $\beta$-ribofuranose in a yield of ca. $96 \%$ as calculated on $\mathrm{N}$-silylated form of 6 ) was dissolved in a mixture of $25 \mathrm{~mL}$ of water and $75 \mathrm{~mL}$ of DCM. The biphasic mixture was cooled in an ice bath at stirring, and $5 \mathrm{~mL}$ of an aqueous solution of freshly prepared sodium borohydride $(0.133 \mathrm{~g}, 0.0035 \mathrm{~mol})$ were added. The reaction mixture was stirred at cooling for 2 hours with monitoring by ${ }^{1} \mathrm{H}$ NMR (acetone- $d_{6}$ ); at the $2 \mathrm{~h}$ time point, the ${ }^{1} \mathrm{H}$ NMR indicated an incomplete reduction of starting compound 6 . Additional $0.266 \mathrm{~g}$ of sodium borohydride in $4 \mathrm{~mL}$ of water were added $\left(0.39 \mathrm{~g}\right.$ of $\mathrm{NaBH}_{4} ; 0.010 \mathrm{~mol}$ in total $)$ and stirring of the reaction mixture was continued at r.t. for $1 \mathrm{~h}$. At this time point, ${ }^{1} \mathrm{H}$ NMR (acetone- $d_{6}$ ) of the reaction mixture indicated a complete reduction of compound 6. Organic phase was separated, washed with brine, dried over $\mathrm{Na}_{2} \mathrm{SO}_{4}$, filtered and evaporated to give a yellow solid foam, $2.30 \mathrm{~g}(85 \%)$. According to ${ }^{1} \mathrm{H}$ NMR, the foam contained a mixture of 1,2-NRH TA, 1,4-NRH TA, and 1,6-NRH TA in a ratio of ca. 0.9:0.8:1.0 (Fig. 24S). To isolate 1,2- and 1,6-isomers of NRH TA, the reaction product was subjected to silica gel column chromatography using a Teledyne chromatography system (RediSep ${ }^{\circledR}$ Flash Column, 40 gram). Fractions were UV monitored at 254 and 350 $\mathrm{nm}$. Chromatography parameters: flow rate, $25 \mathrm{~mL} / \mathrm{min}$; fractions volume, $20 \mathrm{~mL}$. Fraction 1 was eluted with hexanes, fraction 2 was eluted with a 45:55 hexanes/EtOAc mixture, fractions 3-30 were eluted with EtOAc, fractions 31-34 were eluted with a 5:95 EtOH/EtOAc mixture, and further elution was continued with a 10:90 EtOH/EtOAc mixture. 1,2-NRH TA was eluted in fractions 12-16 (UV absorption at $350 \mathrm{~nm}$, Fig. 26S), and after evaporation of the fractions, $0.27 \mathrm{~g}$ (ca. 10\% yield) of 1,2-NRH TA were obtained (portion A). Mixtures of 1,4-NRH TA and 1,6-NRH TA were eluted in fractions 41-46, with the content of 1,6-NRH TA being maximal in fraction 46. UV monitoring of the fractions during chromatography indicated two absorption peaks at $254 \mathrm{~nm}$ which were partially overlapping (Fig. 25S). Based on the absorbance pattern, fractions 41-43 were combined and evaporated to afford $0.51 \mathrm{~g}$ (ca. 19\% yield) of portion B. Fractions 44-46 were combined and evaporated to give $0.38 \mathrm{~g}$ (ca. $14 \%$ yield) of portion C. Each 
of portions A-C was analyzed by ${ }^{1} \mathrm{H}$ NMR (acetone- $d_{6}$ ), and it was found that portion A was composed of 1,2-NRH TA (with some residual DCM and EtOAc being present) (Figs. 26S and 27S). According to integration of the ${ }^{1} \mathrm{H}$ NMR (and ${ }^{13} \mathrm{C}$ NMR) spectra, portion B was composed of a ca. 1.0:0.6 1,4-NRH TA/1,6-NRH TA mixture (Fig. 32S); portion C was composed of a ca. 0.2:1.0 1,4-NRH TA/1,6-NRH TA mixture (Figs. 33S and 34S).

Reduction of NRTA OTf (6) with sodium borohydride in DMF: Synthesis of compounds 8 (1,2-NRH TA) and 9 (1,6-NRH TA). Compound 6 ( $8.50 \mathrm{~g}$; prepared from $2.65 \mathrm{~g}(0.0137 \mathrm{~mol})$ of silylated nicotinamide and $4.35 \mathrm{~g}(0.0137 \mathrm{~mol})$ of 1,2,3,5-tetra- $O$-acetyl-D- $\beta$-ribofuranose in a yield of ca. $100 \%$ as calculated on $\mathrm{N}$-silylated form of 6 ) was dissolved in $55 \mathrm{~mL}$ of anhydrous DMF. The solution was cooled in an ice bath $\left(0^{\circ} \mathrm{C}\right)$ at stirring and a solution of sodium borohydride $(0.54 \mathrm{~g}, 0.0143 \mathrm{~mol})$ in $25 \mathrm{~mL}$ of anhydrous DMF was added to the stirred and cooled solution. The reaction mixture was stirred at cooling for $30 \mathrm{~min}$ and monitored by ${ }^{1} \mathrm{H}$ NMR (acetone- $d_{6}$ ) at this time point, indicating the complete reduction of starting compound 3 and formation of a mixture of 1,2-, 1,4-, and 1,6-isomeric mixture of NRH TA. The reaction was quenched with brine $(\mathrm{ca} .70 \mathrm{~mL})$, and the aqueous phase was repeatedly $(8 \times)$ extracted with EtOAc $\left(500 \mathrm{~mL}\right.$ total volume). The organic phase was washed with brine $(8 \times 40 \mathrm{~mL})$, dried over $\mathrm{Na}_{2} \mathrm{SO}_{4}$, filtered, and evaporated. To remove residual DMF, the product was dissolved in ca. $150 \mathrm{~mL}$ of EtOAc and extracted with brine $(5 \times 30 \mathrm{~mL})$; the organic phase was dried over $\mathrm{Na}_{2} \mathrm{SO}_{4}$, filtered, and evaporated to give yellow solid foam in the amount of $3.61 \mathrm{~g}(69 \%)$. According to ${ }^{1} \mathrm{H}$ NMR, the foam contained a mixture of 1,2-NRH TA, 1,4-NRH TA, and 1,6-NRH TA in the ratio of ca. 1.0:0.44:1.0, respectively (Fig. 40S). To isolate 1,2- and 1,6-isomers of NRH TA, the reaction product was subjected to silica gel column chromatography using Teledyne chromatography system (RediSep ${ }^{\circledR}$ Flash Column 80 gram, catalog \# 692203-380). Fractions were UV monitored at 254 and $350 \mathrm{~nm}$. Chromatography parameters: flow rate, 30 $\mathrm{mL} / \mathrm{min}$; fractions volume $20 \mathrm{~mL}$. Fractions $1-4$ were eluted with hexanes, fractions 5-9 were eluted with a 40:60 hexanes/EtOAc mixture, after which elution was continued with EtOAc (fractions 10-68), 5:95 EtOH/EtOAc (fractions 69-79) and 10:90 EtOH/EtOAc (fractions 80-85) mixtures. 1,2-NRH TA was eluted in fractions 23-35 (UV absorption at $350 \mathrm{~nm}$ ). Fractions 24-28 were combined and evaporated to give portion A $(0.51 \mathrm{~g}, 9.7 \%)$; fractions $29-35$ were combined and evaporated to give portion B $(0.245 \mathrm{~g}$; $4.7 \%$ ). Mixtures of 1,4-NRH TA and 1,6-NRH TA were eluted in fractions 51-85, with last fractions containing mostly 1,6-NRH TA. In particular, fractions 51-58 were combined and evaporated to give portion C (0.404 g, $7.7 \%)$; fractions 59-70 were combined and evaporated to give portion D (0.323 g, 6.2 $\%$ ); fractions 71-79 were combined and evaporated to give portion E (0.226 g, $4.3 \%)$; and fractions 80 85 were combined and evaporated to give portion F $(0.108 \mathrm{~g}, 2.1 \%)$. The total amount of eluted compounds was $1.82 \mathrm{~g}(35 \%)$. Each of portions A-F was analyzed by ${ }^{1} \mathrm{H}$ NMR (acetone- $\left.d_{6}\right)$ and it was found that portions A, B were composed of 1,2-NRH TA (with some residual DCM and EtOAc being present), resulting in the isolated yield of 1,2-NRH TA being equal ca. $0.75 \mathrm{~g}(14.3 \%)$. According to the ${ }^{1} \mathrm{H}$ NMR spectral data (Fig. 41S), portion $\mathrm{C}$ was composed of a ca. 1.0:0.5 1,4-NRH TA/1,6-NRH TA mixture; portion $\mathrm{D}$ was composed of a ca. 0.4:1.0 1,4-NRH TA/1,6-NRH TA mixture; portion $\mathrm{E}$ was composed of a ca. 0.12:1.0 1,4-NRH TA/1,6-NRH TA mixture; and portion E was composed of 1,6-NRH TA, with all the listed portions containing some residual EtOAc. For full characterization, portions $\mathrm{A}+\mathrm{B}$ and $\mathrm{F}$ were analyzed by ${ }^{1} \mathrm{H}$ and ${ }^{13} \mathrm{C}$ NMR (acetone- $d_{6}$ ) (Figs. 42S-45S and Figs. 46S-50S, respectively).

$(2 R, 3 R, 4 R, 5 R)-2$-(acetoxymethyl)-5-(3-carbamoylpyridin-1(2H)-yl)tetrahydrofuran-3,4-diyl

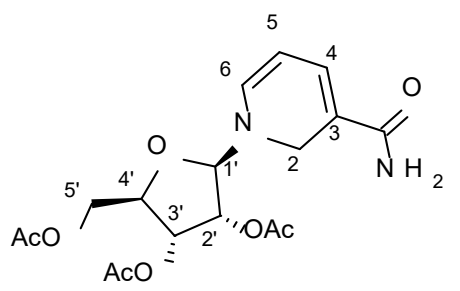
diacetate (8, 1,2-NRH TA). ${ }^{1} \mathrm{H}$ NMR (acetone- $d_{6}, 400 \mathrm{MHz}$ ), $\delta$, ppm: 1.90 (s, 3H, Me), 1.95 (s, 3H, Me), 2.04 (s, 3H, Me), 3.90-3.93 (dd, 1H, $\left.{ }^{3} \mathrm{~J}_{\mathrm{HH}}=13.2 \mathrm{~Hz},{ }^{4} \mathrm{~J}_{\mathrm{HH}}=1.1 \mathrm{~Hz}, 1 / 2 \mathrm{H} 2\right), 4.07-4.10\left(\mathrm{~m}, 2 \mathrm{H}, \mathrm{H} 4^{\prime}+1 / 2 \mathrm{H} 5^{\prime}\right)$, 4.14-4.20 (m, 2H, $\left.1 / 2 \mathrm{H} 2+1 / 2 \mathrm{H} 5^{\prime}\right), 4.76\left(\mathrm{dd}, 1 \mathrm{H},{ }^{3} \mathrm{~J}_{\mathrm{HH}}=6.0 \mathrm{~Hz},{ }^{3} \mathrm{~J}_{\mathrm{HH}}=\right.$ $7.0 \mathrm{~Hz}, \mathrm{H} 5), 4.94\left(\mathrm{~d}, 1 \mathrm{H},{ }^{3} \mathrm{~J}_{\mathrm{HH}}=7.0 \mathrm{~Hz}, \mathrm{H1}{ }^{\prime}\right), 5.15\left(\mathrm{dd},{ }^{3} \mathrm{~J}_{\mathrm{HH}}=2.8 \mathrm{~Hz},{ }^{3} \mathrm{~J}_{\mathrm{HH}}=\right.$ $\left.5.8 \mathrm{~Hz}, 1 \mathrm{H}, \mathrm{H} 3^{\prime}\right), 5.27\left(\mathrm{t}, 1 \mathrm{H},{ }^{3} \mathrm{~J}_{\mathrm{HH}}=6.4 \mathrm{~Hz}, \mathrm{H} 2^{\prime}\right), 6.23\left(\mathrm{br} \mathrm{s}, 2 \mathrm{H}, \mathrm{NH}_{2}\right.$ ), $6.40\left(\mathrm{~d}, 1 \mathrm{H},{ }^{3} \mathrm{~J}_{\mathrm{HH}}=7.2 \mathrm{~Hz}, \mathrm{H} 4\right), 6.58\left(\mathrm{~d}, 1 \mathrm{H},{ }^{3} \mathrm{~J}_{\mathrm{HH}}=5.9 \mathrm{~Hz}, \mathrm{H} 6\right) .{ }^{13} \mathrm{C} \mathrm{NMR}$ 
(acetone- $\left.d_{6}, 100 \mathrm{MHz}\right), \delta$, ppm: $19.46(\mathrm{Me}), 19.63(\mathrm{Me}), 19.83(\mathrm{Me}), 40.81(\mathrm{C} 2), 63.41\left(\mathrm{C}^{\prime}\right), 68.52\left(\mathrm{C} 2{ }^{\prime}\right)$, 71.02 (C3'), $78.72\left(\mathrm{C} 4^{\prime}\right), 94.99$ (C1'), 96.54 (C5), 116.04 (C3), 128.72 (C6), 140.70 (C4), 167.60 (CO), $169.11(\mathrm{CO}), 169.33(\mathrm{CO}), 170.03(\mathrm{CO})$. MS: found $\mathrm{m} / \mathrm{z}=383.07(\mathrm{M}+1)$. HRMS: found $\mathrm{m} / \mathrm{z}=383.1453$; calculated for $\mathrm{M}+1\left(\mathrm{C}_{17} \mathrm{H}_{23} \mathrm{~N}_{2} \mathrm{O}_{8}\right) \mathrm{m} / \mathrm{z}=383.1454$.

$(2 R, 3 R, 4 R, 5 R)-2$-(acetoxymethyl)-5-(5-carbamoylpyridin-1(2H)-yl)tetrahydrofuran-3,4-diyl

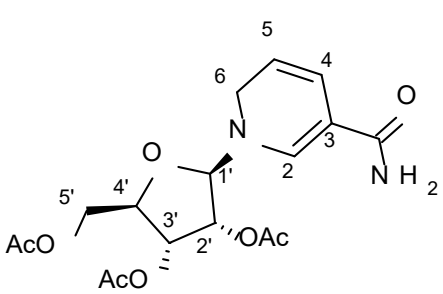
diacetate $\left(9,1,6-N R H\right.$ TA). ${ }^{1} \mathrm{H}$ NMR (acetone- $\left.d_{6}, 400 \mathrm{MHz}\right), \delta$, ppm: 2.04 (s overlapped with acetone- $\left.d_{6}, 3 \mathrm{H}, \mathrm{Me}\right), 2.07(\mathrm{~s}, 3 \mathrm{H}, \mathrm{Me}), 2.08(\mathrm{~s}$, $3 \mathrm{H}, \mathrm{Me}), 4.10-4.12$ (m, 2H, H6), 4.20-4.23 (m, 1H, H4'), 4.23-4.29 (m, $\left.2 \mathrm{H}, \mathrm{H} 5^{\prime}\right), 5.06\left(\mathrm{~d}, 1 \mathrm{H},{ }^{3} \mathrm{~J}_{\mathrm{HH}}=6.7 \mathrm{~Hz}, \mathrm{H1}{ }^{\prime}\right), 5.18\left(\mathrm{dt}, 1 \mathrm{H},{ }^{3} \mathrm{~J}_{\mathrm{HH}}=3.7 \mathrm{~Hz}\right.$, $\left.{ }^{3} \mathrm{~J}_{\mathrm{HH}}=9.9 \mathrm{~Hz}, \mathrm{H} 5\right), 5.23\left(\mathrm{dd}, 1 \mathrm{H},{ }^{3} \mathrm{~J}_{\mathrm{HH}}=3.4 \mathrm{~Hz},{ }^{3} \mathrm{~J}_{\mathrm{HH}}=5.7 \mathrm{~Hz}, \mathrm{H3}{ }^{\prime}\right), 5.36(\mathrm{t}$, $\left.1 \mathrm{H},{ }^{3} \mathrm{~J}_{\mathrm{HH}}=6.3 \mathrm{~Hz}, \mathrm{H} 2^{\prime}\right), 6.09$ (br s, $\left.2 \mathrm{H}, \mathrm{NH}_{2}\right), 6.29\left(\mathrm{dd}, 1 \mathrm{H},{ }^{4} \mathrm{~J}_{\mathrm{HH}}=1.5 \mathrm{~Hz}\right.$, $\left.{ }^{3} \mathrm{~J}_{\mathrm{HH}}=10.0 \mathrm{~Hz}, \mathrm{H} 4\right), 7.24(\mathrm{~s}, 1 \mathrm{H}, \mathrm{H} 2) .{ }^{13} \mathrm{C}$ NMR (acetone- $d_{6}, 100 \mathrm{MHz}$ ), $\delta$, ppm: $19.46(\mathrm{Me}), 19.63(\mathrm{Me}), 19.83(\mathrm{Me}), 42.33(\mathrm{C} 6), 63.24\left(\mathrm{C5}^{\prime}\right)$, $68.63\left(\mathrm{C} 2^{\prime}\right), 70.57\left(\mathrm{C}^{\prime}\right), 78.67\left(\mathrm{C}^{\prime}\right), 95.20\left(\mathrm{C}^{\prime}\right), 104.07(\mathrm{C} 3), 111.91(\mathrm{C} 5), 122.21(\mathrm{C} 4), 142.06(\mathrm{C} 2)$, $167.14(\mathrm{CO}), 169.32(\mathrm{CO}), 169.82(\mathrm{CO}), 169.93(\mathrm{CO})$. MS: found $\mathrm{m} / \mathrm{z}=383.09(\mathrm{M}+1)$. HRMS: found $\mathrm{m} / \mathrm{z}$ $=383.1455$; calculated for $\mathrm{M}+1\left(\mathrm{C}_{17} \mathrm{H}_{23} \mathrm{~N}_{2} \mathrm{O}_{8}\right) \mathrm{m} / \mathrm{z}=383.1454$.

General procedure for the synthesis of 1,2-NRH (2) and 1,6-NRH (3). Under an argon atmosphere and at room temperature, isomeric NRH triacetate (compound $\mathbf{8}$ or 9) $(0.38 \mathrm{~g} ; 1 \mathrm{mmol})$ was dissolved in anhydrous and degassed $\mathrm{MeOH}(7 \mathrm{~mL})$ and $25 \mathrm{wt}$ \% solution of sodium methoxide in methanol $(60 \mu \mathrm{L} ; 0.26 \mathrm{mmol})$ was added at stirring thereto. The reaction solution was stirred under these conditions for ca. $30 \mathrm{~min}$ (TLC control in 10:3 EtOAc/EtOH mixture) and evaporated under reduced pressure at room temperature. Anhydrous DCM was added to the residue and evaporated to afford the corresponding deacetylated product (compound $\mathbf{2}(1,2-\mathrm{NRH})$ or compound $\mathbf{3}(1,6-\mathrm{NRH}))$ as a yellow solid in virtually quantitative yield $(0.26 \mathrm{~g})$.

\section{1-((2R,3R,4S,5R)-3,4-dihydroxy-5-(hydroxymethyl)tetrahydrofuran-2-yl)-1,2-}

dihydropyridine-3-carboxamide (2, 1,2-NRH). ${ }^{1} \mathrm{H}$ NMR $\left(\mathrm{D}_{2} \mathrm{O}, 400 \mathrm{MHz}\right), \delta$, ppm: $3.25(0.78 \mathrm{Me}$ from $\mathrm{MeOH}), 3.60$ and $3.67\left(\mathrm{AB}\right.$ part of $\mathrm{ABX}$ system, $2 \mathrm{H}, \mathrm{J}_{\mathrm{AB}}=12.4 \mathrm{~Hz}, \mathrm{~J}_{\mathrm{BX}}=$<smiles>NC(=O)C1=CC=CN(C2O[C@H](CO)[C@@H](O)[C@H]2O)C1</smiles>
$3.7 \mathrm{~Hz}, \mathrm{~J}_{\mathrm{AX}}=5.0 \mathrm{~Hz}, \mathrm{H}^{\prime}{ }_{\mathrm{A}}$ and $\left.\mathrm{H} 5^{\prime}{ }_{\mathrm{B}}\right), 3.85-3.88\left(\mathrm{~m}, 1 \mathrm{H}, \mathrm{H} 4^{\prime}\right), 3.91(\mathrm{~d}, 1 \mathrm{H}$, $\left.{ }^{3} \mathrm{~J}_{\mathrm{HH}}=12.8 \mathrm{~Hz}, 1 / 2 \mathrm{H} 2\right), 4.02\left(\mathrm{dd}, 1 \mathrm{H},{ }^{3} \mathrm{~J}_{\mathrm{HH}}=3.4 \mathrm{~Hz},{ }^{3} \mathrm{~J}_{\mathrm{HH}}=5.7 \mathrm{~Hz}, \mathrm{H} 3{ }^{\prime}\right), 4.08$ $\left(\mathrm{d}, 1 \mathrm{H},{ }^{3} \mathrm{~J}_{\mathrm{HH}}=12.8 \mathrm{~Hz}, 1 / 2 \mathrm{H} 2\right), 4.19\left(\mathrm{t}, 1 \mathrm{H},{ }^{3} \mathrm{~J}_{\mathrm{HH}}=6.2 \mathrm{~Hz}, \mathrm{H} 2{ }^{\prime}\right), 4.81(\mathrm{~d}$, $\left.{ }^{3} \mathrm{~J}_{\mathrm{HH}}=3.4 \mathrm{~Hz}, 1 \mathrm{H}, \mathrm{H1} 1^{\prime}\right), 5.02\left(\mathrm{t}, 1 \mathrm{H},{ }^{3} \mathrm{~J}_{\mathrm{HH}}=6.6 \mathrm{~Hz}, \mathrm{H} 5\right), 6.57\left(\mathrm{~d}, 1 \mathrm{H},{ }^{3} \mathrm{~J}_{\mathrm{HH}}=\right.$ $7.0 \mathrm{~Hz}, \mathrm{H} 4), 6.77\left(\mathrm{~d}, 1 \mathrm{H},{ }^{3} \mathrm{~J}_{\mathrm{HH}}=6.1 \mathrm{~Hz}, \mathrm{H} 6\right) .{ }^{13} \mathrm{C} \mathrm{NMR}\left(\mathrm{D}_{2} \mathrm{O}, 100 \mathrm{MHz}\right), \delta$, ppm: $40.83(\mathrm{C} 2), 48.83(\mathrm{MeOH}), 61.57\left(\mathrm{C}^{\prime}\right), 69.36\left(\mathrm{C}^{\prime}\right), 70.24\left(\mathrm{C}^{\prime}\right)$, $83.08\left(\mathrm{C} 4^{\prime}\right), 96.48\left(\mathrm{C1}^{\prime}\right), 97.36$ (C5), $111.89(\mathrm{C} 3), 133.22$ (C6), 142.57 (C4), 171.19 (CO). HRMS: found $\mathrm{m} / \mathrm{z}=257.1137$; calculated for $\mathrm{M}+1\left(\mathrm{C}_{11} \mathrm{H}_{17} \mathrm{~N}_{2} \mathrm{O}_{5}\right) \mathrm{m} / \mathrm{z}=257.1132$.

1-((2R,3R,4S,5R)-3,4-dihydroxy-5-(hydroxymethyl)tetrahydrofuran-2-yl)-1,6-

dihydropyridine-3-carboxamide (3, 1,6-NRH). ${ }^{1} \mathrm{H}$ NMR $\left(\mathrm{D}_{2} \mathrm{O}, 400 \mathrm{MHz}\right), \delta$, ppm: 3.25 (1.27 Me from

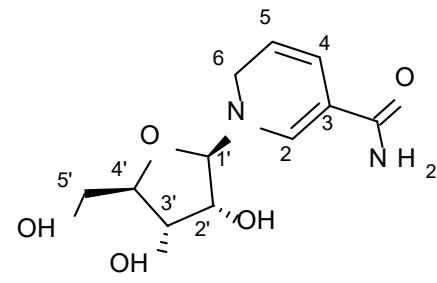
$\mathrm{MeOH}), 3.58$ and $3.66\left(\mathrm{AB}\right.$ part of $\mathrm{ABX}$ system, $2 \mathrm{H}, \mathrm{J}_{\mathrm{AB}}=12.2 \mathrm{~Hz}, \mathrm{~J}_{\mathrm{BX}}=$ $3.9 \mathrm{~Hz}, \mathrm{~J}_{\mathrm{AX}}=5.8 \mathrm{~Hz}, \mathrm{H}^{\prime}{ }_{\mathrm{A}}$ and $\left.\mathrm{H}^{\prime}{ }_{\mathrm{B}}{ }^{\prime}\right), 3.87-3.90\left(\mathrm{~m}, 1 \mathrm{H}, \mathrm{H} 4^{\prime}\right), 3.98(\mathrm{dd}, 1 \mathrm{H}$, $\left.{ }^{3} \mathrm{~J}_{\mathrm{HH}}=3.6 \mathrm{~Hz},{ }^{3} \mathrm{~J}_{\mathrm{HH}}=5.7 \mathrm{~Hz}, \mathrm{H} 3{ }^{\prime}\right), 4.01-4.03(\mathrm{~m}, 2 \mathrm{H}, \mathrm{H} 6), 4.18\left(\mathrm{t}, 1 \mathrm{H},{ }^{3} \mathrm{~J}_{\mathrm{HH}}=\right.$ $\left.6.0 \mathrm{~Hz}, \mathrm{H} 2^{\prime}\right), 4.82\left(\mathrm{~d}, 1 \mathrm{H},{ }^{3} \mathrm{~J}_{\mathrm{HH}}=6.4 \mathrm{~Hz}, \mathrm{H} 1^{\prime}\right), 5.20\left(\mathrm{dt}, 1 \mathrm{H},{ }^{3} \mathrm{~J}_{\mathrm{HH}}=3.7 \mathrm{~Hz}\right.$, $\left.{ }^{3} \mathrm{~J}_{\mathrm{HH}}=10.0 \mathrm{~Hz}, \mathrm{H} 5\right), 6.10\left(\mathrm{dd}, 1 \mathrm{H},{ }^{4} \mathrm{~J}_{\mathrm{HH}}=1.4 \mathrm{~Hz},{ }^{3} \mathrm{~J}_{\mathrm{HH}}=10.0 \mathrm{~Hz}, \mathrm{H} 4\right), 7.24$ $(\mathrm{s}, 1 \mathrm{H}, \mathrm{H} 2) .{ }^{13} \mathrm{C}$ NMR $\left(\mathrm{D}_{2} \mathrm{O}, 100 \mathrm{MHz}\right), \delta, \mathrm{ppm}: 42.37$ (C6), 48.83 $(\mathrm{MeOH}), 61.51\left(\mathrm{C}^{\prime}\right), 69.55\left(\mathrm{C}^{\prime}\right), 70.37\left(\mathrm{C}^{\prime}\right), 83.47\left(\mathrm{C}^{\prime}\right), 97.31\left(\mathrm{Cl}^{\prime}\right)$, 100.42 (C3), 112.84 (C5), 120.54 (C4), 145.18 (C2), 171.44 (CO). HRMS: found $\mathrm{m} / \mathrm{z}=257.1140$; calculated for $\mathrm{M}+1\left(\mathrm{C}_{11} \mathrm{H}_{17} \mathrm{~N}_{2} \mathrm{O}_{5}\right) \mathrm{m} / \mathrm{z}=257.1132$. 
Table S1. ${ }^{1} \mathrm{H}$ NMR data of compounds 7, 8, 9 and 1, $2,3$.

\begin{tabular}{|c|c|c|c|c|c|c|c|c|c|}
\hline \multirow{2}{*}{$\begin{array}{c}\text { Compound } \\
\text { (Solvent) }\end{array}$} & \multicolumn{9}{|c|}{ Chemical shifts, number of protons, multiplicity of peaks (J values in $\mathrm{Hz}$ ) } \\
\hline & $\mathrm{H} 2$ & $\mathrm{H} 4$ & H5 & H6 & H1' & $\mathrm{H} 2{ }^{\prime}$ & H3 ${ }^{\prime}$ & $\mathrm{H} 4{ }^{\prime}$ & $\mathrm{H} 5$ \\
\hline $\begin{array}{c}7 \\
\left(\mathrm{CDCl}_{3}\right)\end{array}$ & $7.09,1 \mathrm{H}, \mathrm{s}$ & $\begin{array}{c}3.06,2 \mathrm{H}, \mathrm{q} \\
(\mathrm{J}=1.4)\end{array}$ & $\begin{array}{l}4.80,1 \mathrm{H}, \mathrm{dt} \\
(\mathrm{J}=3.4 ; 8.2)\end{array}$ & $\begin{array}{l}5.88,1 \mathrm{H}, \mathrm{dd} \\
(\mathrm{J}=1.7 ; 8.2)\end{array}$ & $\begin{array}{c}4.89,1 \mathrm{H}, \mathrm{d} \\
(\mathrm{J}=7.0)\end{array}$ & $\begin{array}{c}5.11,1 \mathrm{H}, \mathrm{t} \\
(\mathrm{J}=6.4)\end{array}$ & $\begin{array}{l}5.18,1 \mathrm{H}, \mathrm{dd} \\
(\mathrm{J}=2.8 ; 5.8)\end{array}$ & $\begin{array}{l}4.11,1 \mathrm{H}, \mathrm{dd} \\
(\mathrm{J}=3.2 ; 4.7)\end{array}$ & $4.20-4.21,2 \mathrm{H}, \mathrm{m}$ \\
\hline $\begin{array}{c}\mathbf{8} \\
\left(\text { acetone- } \mathrm{d}_{6} \text { ) }\right.\end{array}$ & $\begin{array}{c}3.90-3.93,1 \mathrm{H}(1 / 2 \mathrm{H} 2) \\
\mathrm{dd} \\
(\mathrm{J}=1.1 ; 13.2) \\
4.14-4.20,{ }^{*} 1 \mathrm{H} \\
(1 / 2 \mathrm{H} 2), \mathrm{m}\end{array}$ & $\begin{array}{l}6.40,1 \mathrm{H}, \mathrm{d} \\
\quad(\mathrm{J}=7.2)\end{array}$ & $\begin{array}{l}4.76,1 \mathrm{H}, \mathrm{dd} \\
(\mathrm{J}=6.0 ; 7.0)\end{array}$ & $\begin{array}{l}6.58,1 \mathrm{H}, \mathrm{d} \\
\quad(\mathrm{J}=5.9)\end{array}$ & $\begin{array}{l}4.94,1 \mathrm{H}, \mathrm{d} \\
(\mathrm{J}=7.0)\end{array}$ & $\begin{array}{c}5.27,1 \mathrm{H}, \mathrm{t} \\
(\mathrm{J}=6.4)\end{array}$ & $\begin{array}{l}5.15,1 \mathrm{H}, \mathrm{dd} \\
(\mathrm{J}=2.8 ; 5.8)\end{array}$ & $4.07-4.10,2 \mathrm{H}$ & $\left(\mathrm{H} 4^{\prime}+1 / 2 \mathrm{H} 5^{\prime}\right), \mathrm{m}$ \\
\hline $\begin{array}{c}9 \\
\text { (acetone- } \mathrm{d}_{6} \text { ) } \\
\end{array}$ & $7.24,1 \mathrm{H}, \mathrm{s}$ & $\begin{array}{c}6.29,1 \mathrm{H}, \mathrm{dd} \\
(\mathrm{J}=1.5 ; 10.0)\end{array}$ & $\begin{array}{l}5.18,1 \mathrm{H}, \mathrm{dt} \\
(\mathrm{J}=3.7 ; 9.9)\end{array}$ & $\begin{array}{c}4.10-4.12,2 \mathrm{H}, \\
\mathrm{m}\end{array}$ & $\begin{array}{c}5.06,1 \mathrm{H}, \mathrm{d} \\
(\mathrm{J}=6.7)\end{array}$ & $\begin{array}{c}5.36,1 \mathrm{H}, \mathrm{t} \\
(\mathrm{J}=6.3)\end{array}$ & $\begin{array}{l}5.23,1 \mathrm{H}, \mathrm{dd} \\
(\mathrm{J}=3.4 ; 5.7)\end{array}$ & $\begin{array}{c}4.20-4.23 \\
1 \mathrm{H}, \mathrm{m}\end{array}$ & $4.23-4.29,2 \mathrm{H}, \mathrm{m}$ \\
\hline $\begin{array}{c}1 \\
\left(\mathrm{D}_{2} \mathrm{O}\right)\end{array}$ & $7.06,1 \mathrm{H}, \mathrm{s}$ & $\begin{array}{l}2.98,2 \mathrm{H}, \mathrm{q} \\
\quad(\mathrm{J}=1.5)\end{array}$ & $\begin{array}{l}4.90,1 \mathrm{H}, \mathrm{dt} \\
(\mathrm{J}=3.4 ; 8.2)\end{array}$ & $\begin{array}{l}6.01,1 \mathrm{H}, \mathrm{dd} \\
(\mathrm{J}=1.5 ; 8.2)\end{array}$ & $\begin{array}{l}4.79,1 \mathrm{H}, \mathrm{d} \\
(\mathrm{J}=7.0)\end{array}$ & $\begin{array}{l}4.11,1 \mathrm{H}, \mathrm{t} \\
(\mathrm{J}=6.3)\end{array}$ & $\begin{array}{l}4.04,1 \mathrm{H}, \mathrm{dd} \\
(\mathrm{J}=2.9 ; 5.6)\end{array}$ & $\begin{array}{l}3.88,1 \mathrm{H}, \mathrm{dd} \\
(\mathrm{J}=3.5 ; 6.8)\end{array}$ & $\begin{array}{c}3.60,3.66,2 \mathrm{H}, \\
\text { AB of ABX } \\
(\mathrm{J}=12.5 ; 3.6 ; 4.8)\end{array}$ \\
\hline $\begin{array}{c}2 \\
\left(\mathrm{D}_{2} \mathrm{O}\right)\end{array}$ & $\begin{array}{l}3.91,1 \mathrm{H}(1 / 2 \mathrm{H} 2), \mathrm{d} \\
(\mathrm{J}=12.8) \\
4.08,1 \mathrm{H}(1 / 2 \mathrm{H}), \mathrm{d} \\
\quad(\mathrm{J}=12.8)\end{array}$ & $\begin{array}{l}6.57,1 \mathrm{H}, \mathrm{d} \\
\quad(\mathrm{J}=7.0)\end{array}$ & $\begin{array}{c}5.02,1 \mathrm{H}, \mathrm{t} \\
(\mathrm{J}=6.6)\end{array}$ & $\begin{array}{l}6.77,1 \mathrm{H}, \mathrm{d} \\
\quad(\mathrm{J}=6.1)\end{array}$ & $\begin{array}{l}4.81,1 \mathrm{H}, \mathrm{d} \\
\quad(\mathrm{J}=3.4)\end{array}$ & $\begin{array}{l}4.19,1 \mathrm{H}, \mathrm{t} \\
(\mathrm{J}=6.2)\end{array}$ & $\begin{array}{l}4.02,1 \mathrm{H}, \mathrm{dd} \\
(\mathrm{J}=3.4 ; 5.7)\end{array}$ & $\begin{array}{c}3.85-3.88 \\
1 \mathrm{H}, \mathrm{m}\end{array}$ & $\begin{array}{c}3.60,3.67,2 \mathrm{H}, \\
\text { AB of ABX } \\
(\mathrm{J}=12.4 ; 3.7 ; 5.0)\end{array}$ \\
\hline $\begin{array}{c}3 \\
\left(\mathrm{D}_{2} \mathrm{O}\right)\end{array}$ & $7.24,1 \mathrm{H}, \mathrm{s}$ & $\begin{array}{c}6.10,1 \mathrm{H}, \mathrm{dd} \\
(\mathrm{J}=1.4 ; 10.0)\end{array}$ & $\begin{array}{c}5.20,1 \mathrm{H}, \mathrm{dt} \\
(\mathrm{J}=3.7 ; 10.0)\end{array}$ & $\begin{array}{c}4.01-4.03,2 \mathrm{H} \\
\mathrm{m}\end{array}$ & $\begin{array}{l}4.82,1 \mathrm{H}, \mathrm{d} \\
\quad(\mathrm{J}=6.4)\end{array}$ & $\begin{array}{l}4.18,1 \mathrm{H}, \mathrm{t} \\
(\mathrm{J}=6.0)\end{array}$ & $\begin{array}{l}3.98,1 \mathrm{H}, \mathrm{dd} \\
(\mathrm{J}=3.6 ; 5.7)\end{array}$ & $\begin{array}{c}3.87-3.90 \\
1 \mathrm{H}, \mathrm{m}\end{array}$ & $\begin{array}{c}3.58,3.66,2 \mathrm{H}, \\
\text { AB of ABX } \\
(\mathrm{J}=12.2 ; 3.9 ; 5.8)\end{array}$ \\
\hline
\end{tabular}

* Overlapped with $1 / 2 \mathrm{H} 5$ ' 
Table S2. ${ }^{3} \mathrm{C}$ NMR data of compounds 7, 8, 9 and 1, 2, 3.

\begin{tabular}{|c|c|c|c|c|c|c|c|c|c|c|}
\hline \multirow{2}{*}{$\begin{array}{c}\text { Compound } \\
\text { (Solvent) }\end{array}$} & \multicolumn{10}{|c|}{ Chemical shifts } \\
\hline & $\mathrm{C} 2$ & $\mathrm{C} 3$ & $\mathrm{C} 4$ & $\mathrm{C} 5$ & C6 & $\mathrm{C} 1$ ' & $\mathrm{C} 2$ ' & $\mathrm{C} 3$, & C4' & $\mathrm{C} 5$ \\
\hline $\begin{array}{c}7 \\
\left(\mathrm{CDCl}_{3}\right)\end{array}$ & 134.36 & 100.38 & 21.22 & 102.49 & 123.07 & 91.36 & \multicolumn{2}{|c|}{$68.87,68.91$} & 77.05 & 61.60 \\
\hline $\begin{array}{c}\mathbf{8} \\
\left.\text { (acetone- } \mathrm{d}_{6}\right) \\
\end{array}$ & 40.81 & 116.04 & 140.70 & 96.54 & 128.72 & 94.99 & 68.52 & 71.02 & 78.72 & 63.41 \\
\hline $\begin{array}{c}9 \\
\left.\text { (acetone- } \mathrm{d}_{6}\right)\end{array}$ & 142.06 & 104.07 & 122.21 & 111.91 & 42.33 & 95.20 & 68.63 & 70.57 & 78.67 & 63.24 \\
\hline $\begin{array}{c}1 \\
\left(\mathrm{D}_{2} \mathrm{O}\right)\end{array}$ & 137.80 & 101.08 & 22.07 & 105.20 & 125.37 & 95.03 & 71.11 & 70.21 & 85.36 & 61.63 \\
\hline $\begin{array}{c}\mathbf{2} \\
\left(\mathrm{D}_{2} \mathrm{O}\right)\end{array}$ & 40.83 & 111.89 & 142.57 & 97.36 & 133.22 & 96.48 & 69.36 & 70.24 & 83.08 & 61.57 \\
\hline $\begin{array}{c}\mathbf{3} \\
\left(\mathrm{D}_{2} \mathrm{O}\right)\end{array}$ & 145.18 & 100.42 & 120.54 & 112.84 & 42.37 & 97.31 & 69.55 & 70.37 & 83.47 & 61.51 \\
\hline
\end{tabular}


Isomerism of NRH and $\mathrm{NR}^{+}$:

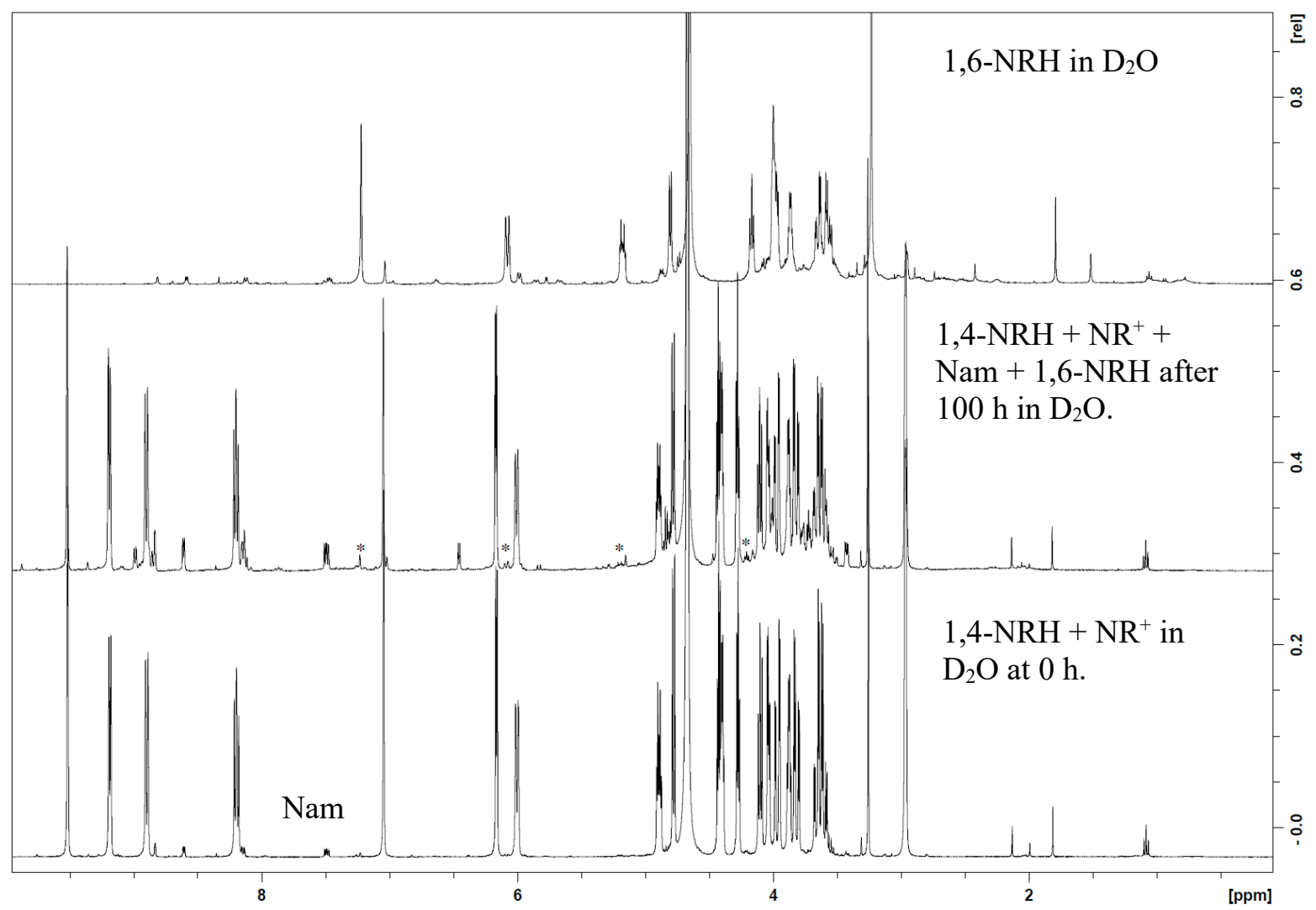

Fig. S1: ESI-NMR1: 1,4-NRH was co-incubated with $\mathrm{NR}^{+}$in $\mathrm{D}_{2} \mathrm{O}$ (final concentration $10 \mathrm{mM}$ of each component). Under these conditions, the 1,6-NRH isomer formed and could be detected after $100 \mathrm{~h}$ as indicated by $\mathrm{a} *$.

Products that can readily be identified include alpha and beta-ribose, nicotinamide, $\mathrm{NR}^{+}$and 1,6-NRH. The appearance of products with aliphatic hydrogens consistent with structures proposed by Margoli via the intermediacy of $\mathrm{NR}(\mathrm{OH}) \mathrm{H}$, which can also generate decomposition products as proposed in the scheme below, which remain to be characterized. 
Figure S2: Buffer conditions affect the stability of 1,2-, 1,4- and 1,6-NRH in solution in absence of NQO2.

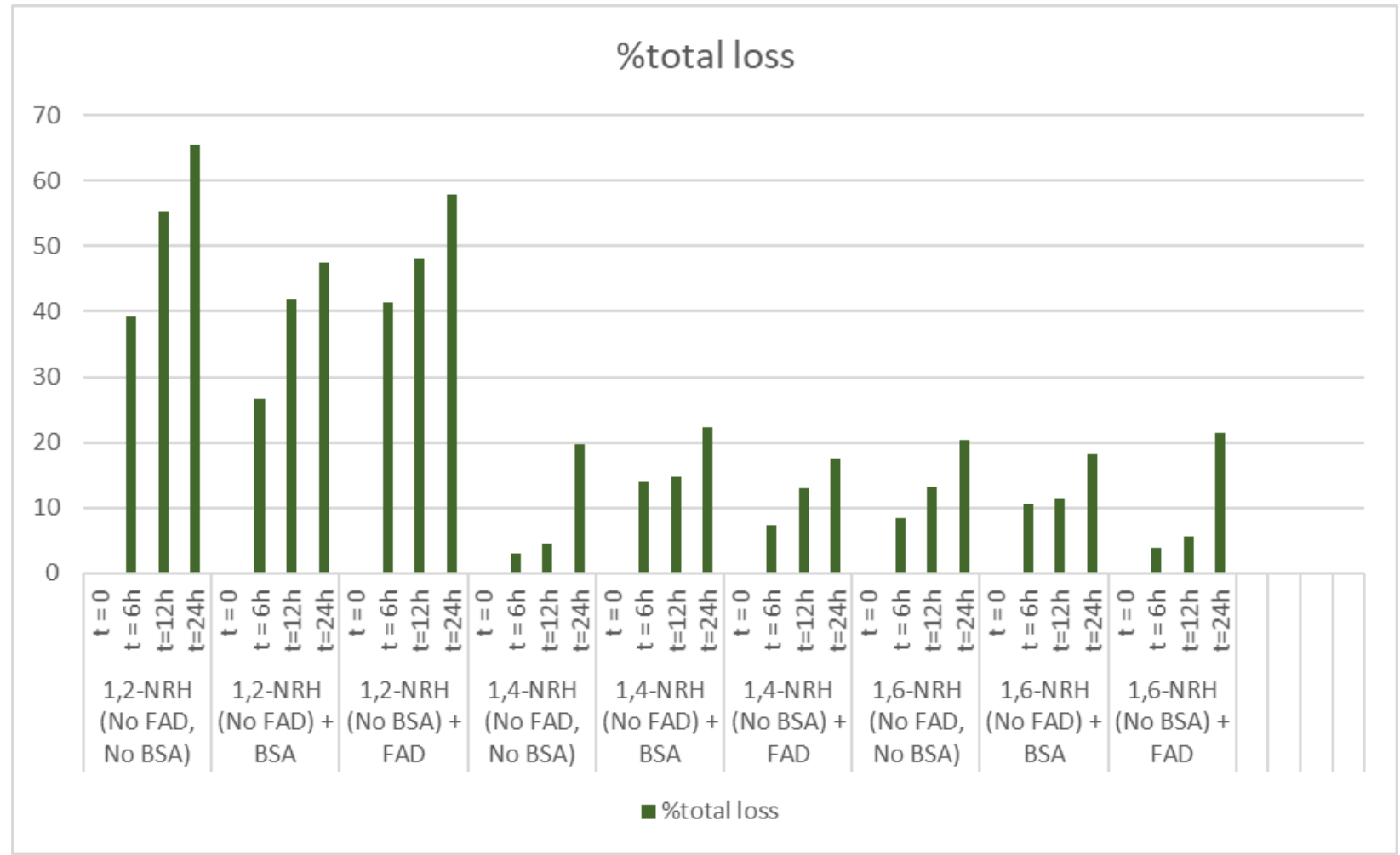

Figure S3: Buffer conditions affect the product distribution of 1,6-NRH in solution in absence of NQO2.

\section{1,6-NRH stability to BSA and FAD}

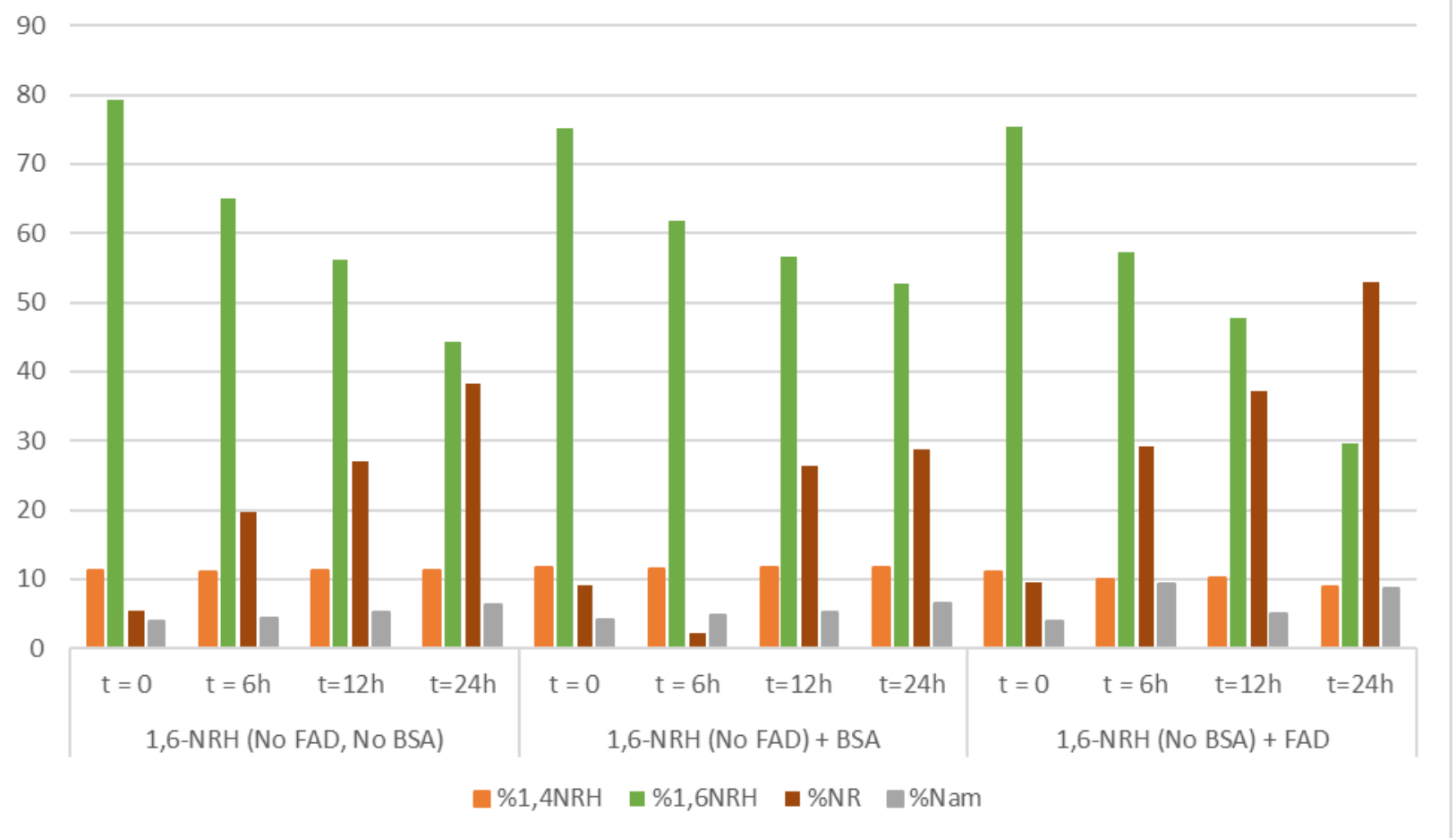


Figure S4: Changes in the product distribution of 1, 2-, 1,4- and 1,6-NRH in solution in absence and presence of NQO2 measured by ${ }^{1} \mathrm{H}$ NMR.
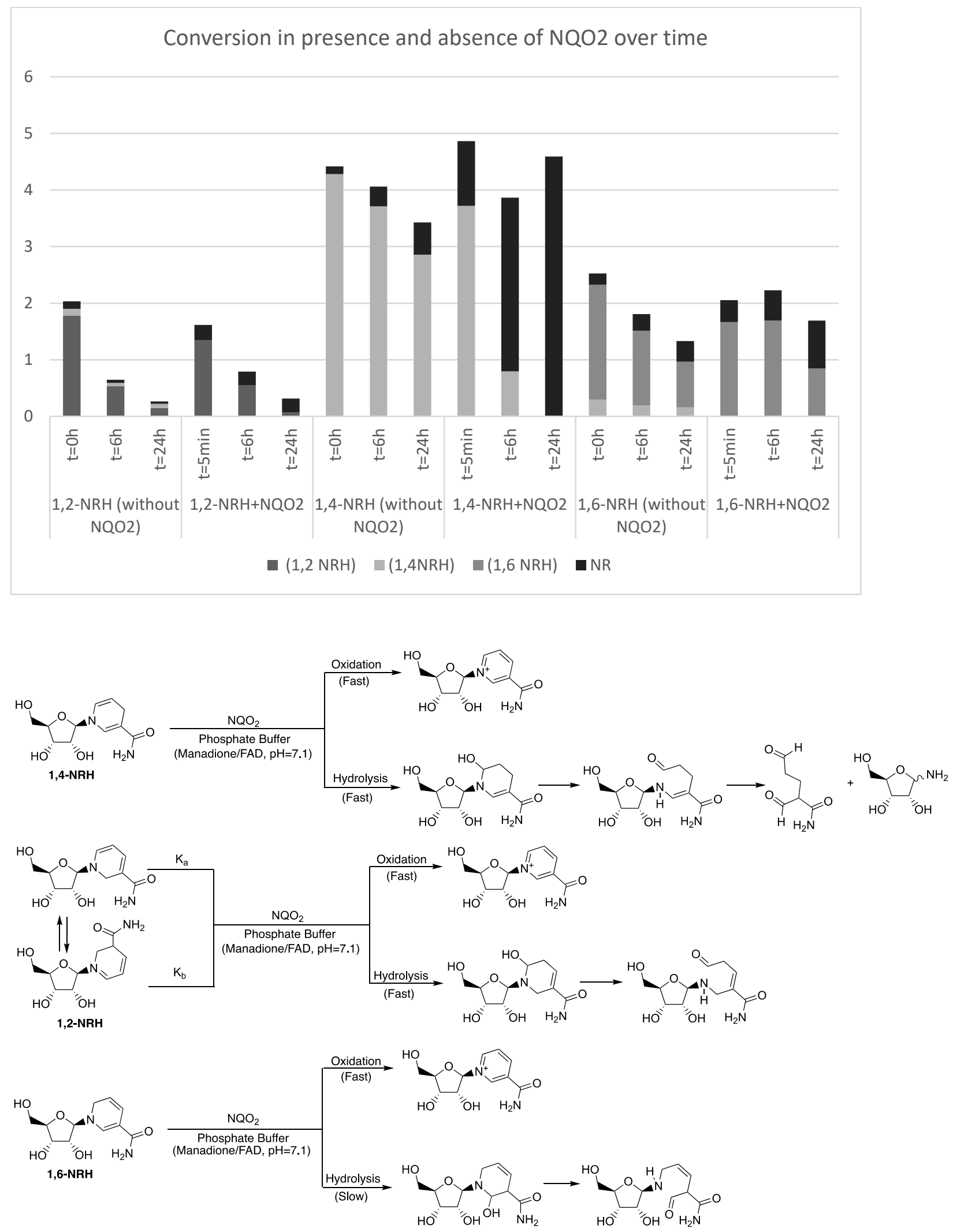

Scheme S1: Stability and reactivity of the three dihydronicotinamide ribosides under NQO2 enzymatic conditions. 
Cytotoxicity studies.

\begin{tabular}{|c|c|c|c|c|}
\multicolumn{5}{|c}{ HEK293T } \\
\hline Samples & Cell & $1,4-\mathrm{NRH}$ & $1,6-\mathrm{NRH}$ & $1,2-\mathrm{NRH}$ \\
\hline$\%$ Survival & 100.00 & 88.97 & 86.01 & 88.01 \\
\hline$\%$ SEM & 0.00 & 2.14 & 2.51 & 2.92 \\
\hline
\end{tabular}

\begin{tabular}{|c|c|c|c|c|}
\multicolumn{5}{|c}{ HepG3 } \\
\hline Samples & Cell & $1,4-\mathrm{NRH}$ & $1,6-\mathrm{NRH}$ & $1,2-\mathrm{NRH}$ \\
\hline$\%$ Survival & 100.00 & 92.45 & 92.68 & 97.41 \\
\hline$\%$ SEM & 0.00 & 0.75 & 1.95 & 1.82 \\
\hline
\end{tabular}

Modified peptides identified with high confidence and calculated abundances (based on peak area):

\begin{tabular}{|c|c|c|c|}
\hline Sequence & Modifications & Abundances:BSA & $\begin{array}{l}\text { Abundances: } \\
\text { BSA + NRH }\end{array}$ \\
\hline LHEKTPVSEKVTK & NRH Adduct C6H9NO2 [K4] & $2.61 \mathrm{E}+08$ & $1.1 \mathrm{E}+09$ \\
\hline SQKFPKAEFVEVTK & NRH Adduct C6H9NO2 [K] & $1.42 E+08$ & $1.84 \mathrm{E}+08$ \\
\hline QEAKDAFLGSFLYEYSR & $\mathrm{NRH}[\mathrm{K} 4]$ & 11990748 & 15794084 \\
\hline CVLHEKTPVSEKVTK & NRH Adduct C6H9NO2 [K6] & $4.39 \mathrm{E}+08$ & 2.97E+08 \\
\hline HPYFYAPELLYYANKYNGVFQECCQAEDKGACLLP & NRH Adduct $\mathrm{C} 6 \mathrm{H} 9 \mathrm{NO} 2[\mathrm{~K}]$ & 68868568 & 16978286 \\
\hline CTKPESERMPCTEDYLSLILNR & NRH Adduct C11H18N2O5 [K3] & $1.87 \mathrm{E}+08$ & 37090620 \\
\hline HKDDSPDLPK & NRH Adduct C11H18N2O6 [K2] & 16943046 & 28272632 \\
\hline CTKPESERMPCTEDYLSLILNR & NRH Adduct C6H9NO3 [K3] & & $6.46 \mathrm{E}+08$ \\
\hline YQEAKDAFLGSFLYEYSR & NRH Adduct C11H18N2O5 [K5] & 8007322 & 16282244 \\
\hline CDNQDTISSKLKECCDKPLLEK & NRH Adduct C11H18N2O6 [K12] & $2.15 E+08$ & $1.35 E+08$ \\
\hline HLVDEPQNLIKQNCDQF & NRH Adduct C11H18N2O5 [K11] & 46415816 & 25779761 \\
\hline KECCDKPLLEK & NRH Adduct C6H9NO2 [K1] & 49370352 & 51988600 \\
\hline LHEKTPVSEK & NRH Adduct C6H9NO2 [K4] & 32803514 & 88550358 \\
\hline QKFPKAEFVEVTK & $\begin{array}{l}\text { NRH Adduct C6H9NO2 [K]; NRH } \\
\text { Adduct C6H9NO3 [K] }\end{array}$ & $2.7 \mathrm{E}+08$ & $2.73 E+08$ \\
\hline LEECCAKDDPHACYSTVFDKLK & $\mathrm{NRH}[\mathrm{K} 7]$ & 30435586 & 12151486 \\
\hline ADESHAGCEKSLHTLFGDELCK & NRH Adduct C6H9NO2 [K10] & 23741036 & 36932476 \\
\hline TMREKVLASSAR & NRH Adduct C11H18N2O5 [K5] & 2958651 & 1968951 \\
\hline PKIETMREK & $\mathrm{NRH}[\mathrm{K} 2]$ & 17969050 & 48940296 \\
\hline
\end{tabular}

Identified PSMs from BSA without NRH that contain a modification:

High Confidence, Modified Protein Coverage: $37.23 \%$

(94.56\% Total coverage regardless of modification)

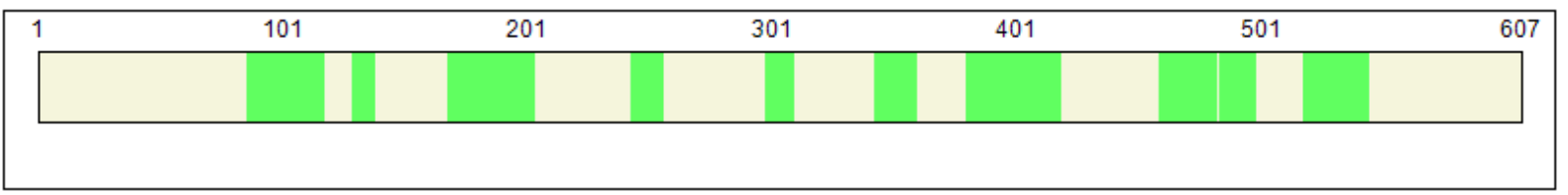

\section{Found Modifications:}

$\begin{array}{ll}\text { C } & \text { Custom: NRH Adduct C6H9NO2 (K) } \\ \text { D } & \text { Custom: NRH Adduct C11H18N2O6 (K) } \\ \text { E } & \text { Custom: NRH Adduct C11H18N2O5 (K) } \\ \text { F } & \text { Custom: NRH Adduct C6H9NO3 (K) } \\ \text { G } & \text { Custom:NRH (K) }\end{array}$

MKWVTFISLL LLFSSAYSRG VFRRDTHKSE IAHRFKDLGE EHFKGLVLIA FSQYLQQCPF DEHVKLVNEL

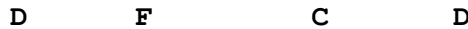

TEFAKTCVAD ESHAGCEKSL HTLFGDELCK VASLRETYGD MADCCEKQEP ERNECFLSHK DDSPDLPKLK $\mathrm{E}$
$\mathrm{C}$

PDPNTLCDEF KADEKKFWGK YLYEIARRHP YFYAPELLYY ANKYNGVFQE CCQAEDKGAC LLPKIETMRE 
C C

KVLASSARQR LRCASIQKFG ERALKAWSVA RLSQKFPKAE FVEVTKLVTD LTKVHKECCH GDLLECADDR F

ADLAKYICDN QDTISSKLKE CCDKPLLEKS HCIAEVEKDA IPENLPPLTA DFAEDKDVCK NYQEAKDAFL

G $\mathbf{F}$

GSFLYEYSRR HPEYAVSVLL RLAKEYEATL EECCAKDDPH ACYSTVFDKL KHLVDEPQNL IKQNCDQFEK G

LGEYGFQNAL IVRYTRKVPQ VSTPTLVEVS RSLGKVGTRC CTKPESERMP CTEDYLSLIL NRLCVLHEKT

$\begin{array}{ll}\mathbf{F} & \mathbf{G} \\ \mathbf{E} & \mathbf{F} \\ \mathbf{D} & \mathbf{E}\end{array}$

PVSEKVTKCC TESLVNRRPC FSALTPDETY VPKAFDEKLF TFHADICTLP DTEKQIKKQT ALVELLKHKP KATEEQLKTV MENFVAFVDK CCAADDKEAC FAVEGPKLVV STQTALA

Identified PSMs from BSA + NRH that contain a modification:

High Confidence, Modified Protein Coverage: $46.79 \%$

( $95.72 \%$ Total coverage regardless of modification)

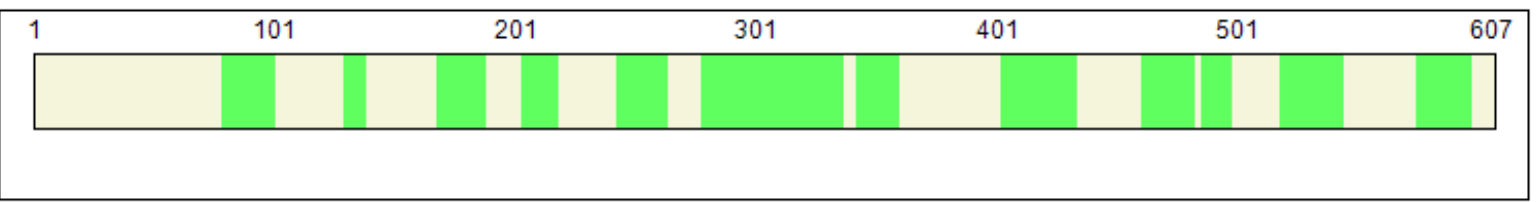

Found Modifications:

C Custom: NRH Adduct C6H9NO2 (K)

D Custom: NRH Adduct $\mathrm{C} 11 \mathrm{H} 18 \mathrm{~N} 2 \mathrm{O} 6(\mathrm{~K})$

E Custom: $\mathrm{NRH}$ Adduct $\mathrm{C} 11 \mathrm{H} 18 \mathrm{~N} 2 \mathrm{O} 5(\mathrm{~K})$

F Custom: NRH Adduct C6H9NO3 (K)

G Custom:NRH (K)

MKWVTFISLL LLFSSAYSRG VFRRDTHKSE IAHRFKDLGE EHFKGLVLIA FSQYLQQCPF DEHVKLVNEL D $\quad E$

TEFAKTCVAD ESHAGCEKSL HTLFGDELCK VASLRETYGD MADCCEKQEP ERNECFLSHK DDSPDLPKLK G

G

PDPNTLCDEF KADEKKFWGK YLYEIARRHP YFYAPELLYY ANKYNGVFQE CCQAEDKGAC LLPKIETMRE $\begin{array}{lll}C & \text { F } & \text { F } \\ \text { C } & \text { D D } & \text { C }\end{array}$

KVLASSARQR LRCASIQKFG ERALKAWSVA RLSQKFPKAE FVEVTKLVTD LTKVHKECCH GDLLECADDR E G

G

$\begin{array}{lllllllllll}F & \text { D } & \text { C } & G & \text { E } & & \text { E } & \text { C }\end{array}$

ADLAKYICDN QDTISSKLKE CCDKPLLEKS HCIAEVEKDA IPENLPPLTA DFAEDKDVCK NYQEAKDAFL C C

GSFLYEYSRR HPEYAVSVLL RLAKEYEATL EECCAKDDPH ACYSTVFDKL KHLVDEPQNL IKQNCDQFEK $\mathrm{D}$

F

C

LGEYGFQNAL IVRYTRKVPQ VSTPTLVEVS RSLGKVGTRC CTKPESERMP CTEDYLSLIL NRLCVLHEKT E $\quad \mathbf{G}$

C $\mathbf{F}$

PVSEKVTKCC TESLVNRRPC FSALTPDETY VPKAFDEKLF TFHADICTLP DTEKQIKKQT ALVELLKHKP C $\stackrel{\text { D }}{ } \stackrel{\text { D }}{ }$

KATEEQLKTV MENFVAFVDK CCAADDKEAC FAVEGPKLVV STQTALA 
Quantum-based Computational Modeling NQO2 ${ }^{1-9}$.

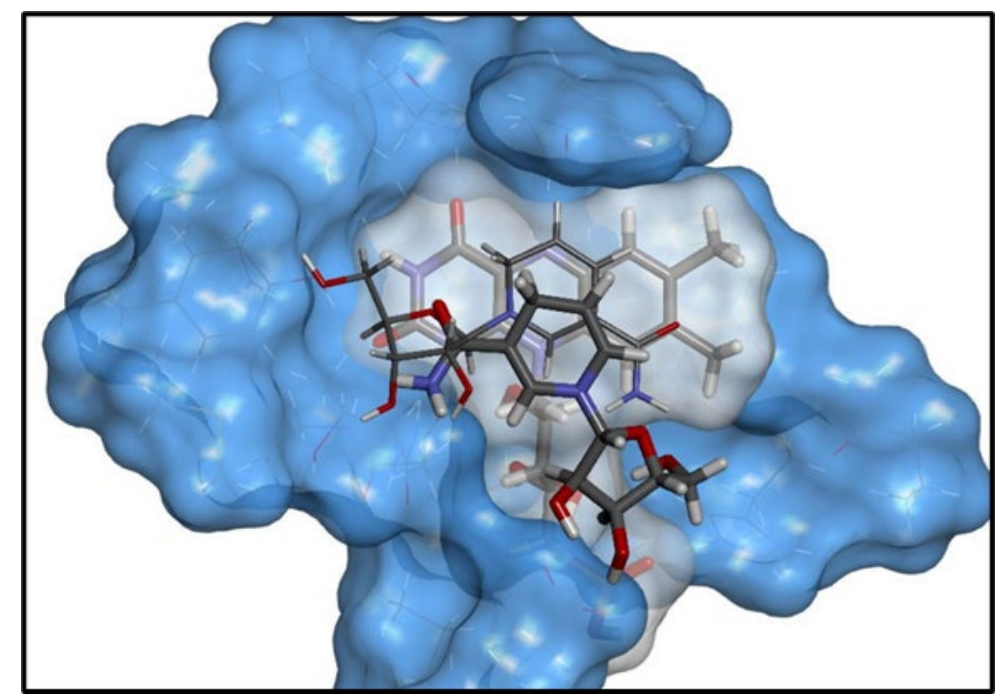

Figure S5: Superposition of 1,4- and 1,6-NRH bound to the face of the flavin moiety of FAD (transparent surface) in FAD-bound NQO2 (blue surface). The 1,4- and 1,6-NRH isomers are represented as thick and thin stick structures, respectively. The computational model of FAD-bound NQO2 is based upon PDB entry 2QX847.

A truncated model of the NQO2(FAD) enzyme (17 amino acid residues) was built from PDB entry 2QX8[1] (res $1.60 \AA$ ) using Gaussview 5.0 (Gaussian Inc, Wallingford, CT). The FAD molecule was truncated at the first phosphate to produce a model system with a net charge of -1 . In the search for favorable docking poses of the three NRH isomers on the face of FAD, 8 classes of poses of each isomer were considered through manual positioning: 2 positions for the amide moiety $\mathrm{x} 2$ positions for the glycosidic bond $\mathrm{x} 2$ positions for the ribose moiety (left versus right of FAD). Manual docking was followed by semi-empirical PM7 energy optimizations in which only the NRH ligand was permitted to move. The resulting lowest energy structures were selected for follow-up ONIOM[2] hybrid calculations (high-level:B3LYP[3,4]/6-31G(d)[5-7]:lowlevel:PM7) in order to distinguish them by using an all-electron density functional model (B3LYP) for the high-level region. The ligand positions were reoptimized, ultimately using the ONIOM(wB97XD/6$31 \mathrm{G}(\mathrm{d}):$ PM7) model in the presence of water using the default SCRF model. The wB97XD model[8] is a density functional method that includes the effects of dispersion. Optimizations were deemed complete when the forces met the default convergence criteria. All calculations were carried out using Gaussian16[9].

1. Calamini, B.; Santarsiero, B.D.; Boutin, J.A.; Mesecar, A.D. Kinetic, thermodynamic and X-ray structural insights into the interaction of melatonin and analogues with quinone reductase 2. Biochem. J. 2008, 413, 81-91.

2. Dapprich, S.; Komáromi, I.; Byun, K.; Morokuma, K.; Frisch, M.J. A new ONIOM implementation in Gaussian98. Part I. The calculation of energies, gradients, vibrational frequencies and electric field derivatives. J. Mol. Struct. THEOCHEM 1999, 461, $1-21$.

3. Becke, A.D. Density-functional thermochemistry. III. The role of exact exchange. J. Chem. Phys. 1993, 98, 5648-5652.

4. Lee, C.; Yang, W.; Parr, R.G. Development of the Colle-Salvetti correlation-energy formula into a functional of the electron density. Phys. Rev. B 1988, 37, 785-789.

5. Ditchfield, R. Self-Consistent Molecular-Orbital Methods. IX. An Extended Gaussian-Type Basis for Molecular-Orbital Studies of Organic Molecules. J. Chem. Phys. 1971, 54, 724.

6. Hehre, W.J. Self-Consistent Molecular Orbital Methods. XII. Further Extensions of Gaussian-Type Basis Sets for Use in Molecular Orbital Studies of Organic Molecules. J. Chem. Phys. 1972, 56, 2257.

7. Rassolov, V.A.; Windus, T.L.; Pople, J.A.; Ratner, M.A. 6-31G* basis set for atoms K through Zn. J. Chem. Phys. 1998, 109, $1223-1229$.

8. Chai, J.-D.; Head-Gordon, M. Long-range corrected hybrid density functionals with damped atom-atom dispersion corrections. Phys. Chem. Chem. Phys. 2008, 10, 6615-20.

9. Frisch, M.J.; Trucks, G.W.; Schlegel, H.B.; Scuseria, G.E.; Robb, M.A.; Cheeseman, J.R.; Scalmani, G.; Barone, V.; Petersson, G.A.; Nakatsuji, H.; et al. Gaussian 16, Revision C.01; Gaussian, Inc.: Wallingford, CT, USA, 2016. 
Spectra

\begin{tabular}{|c|c|}
\hline NMR, MS and HRMS data & 1 \\
\hline Fig. 3S ${ }^{1} \mathrm{H}$ NMR $\left(\mathrm{C}_{6} \mathrm{D}_{6}\right)$ of $N$-(trimethylsilyl)Nam. & 3 \\
\hline Fig. $4 \mathrm{~S}^{13} \mathrm{C}$ NMR $\left(\mathrm{C}_{6} \mathrm{D}_{6}\right)$ of $N$-(trimethylsilyl)Nam. & 4 \\
\hline Fig. 5S ${ }^{1} \mathrm{H}$ NMR $\left(\mathrm{D}_{2} \mathrm{O}\right)$ of NR triacetate triflate $(6)$. & 5 \\
\hline Fig. 6S ${ }^{19} \mathrm{~F}$ NMR $\left(\mathrm{D}_{2} \mathrm{O}\right)$ of NR triacetate triflate $(6)$. & 6 \\
\hline Fig. $7 \mathrm{~S}^{13} \mathrm{C}$ NMR $\left(\mathrm{D}_{2} \mathrm{O}\right)$ of NR triacetate triflate $(6)$. & 7 \\
\hline Fig. 8S ${ }^{1} \mathrm{H}-{ }^{1} \mathrm{H}$ correlation $(\mathrm{COSY}) \mathrm{NMR}\left(\mathrm{D}_{2} \mathrm{O}\right)$ of NR triacetate triflate $(6)$. & 8 \\
\hline Fig. 9S ${ }^{1} \mathrm{H}-{ }^{13} \mathrm{C}$ correlation (HSQC) NMR $\left(\mathrm{D}_{2} \mathrm{O}\right)$ of NR triacetate triflate $(6)$. & 9 \\
\hline Fig. 10S MS $\left(1: 1 \mathrm{H}_{2} \mathrm{O} / \mathrm{ACN}\right)$ of NRTA triflate (6). & 10 \\
\hline Fig. 11S HRMS $\left(1: 1 \mathrm{H}_{2} \mathrm{O} / \mathrm{ACN}\right)$ of NRTA triflate (6). & 11 \\
\hline Fig. 12S ${ }^{1} \mathrm{H}$ NMR $\left(\mathrm{CDCl}_{3}\right)$ of $\mathrm{NRH}$ triacetate $(7)$. & 12 \\
\hline Fig. 13S ${ }^{13} \mathrm{C} \mathrm{NMR}\left(\mathrm{CDCl}_{3}\right)$ of NRH triacetate (7). & 13 \\
\hline Fig. 14S ${ }^{1} \mathrm{H}-{ }^{1} \mathrm{H}$ correlation $(\mathrm{COSY}) \mathrm{NMR}\left(\mathrm{CDCl}_{3}\right)$ of $\mathrm{NRH}$ triacetate $(7)$. & 14 \\
\hline Fig. 15S ${ }^{1} \mathrm{H}^{-1}{ }^{13} \mathrm{C}$ correlation (HSQC) NMR $\left(\mathrm{CDCl}_{3}\right)$ of $\mathrm{NRH}$ triacetate (7). & 15 \\
\hline Fig. 16S MS $\left(1: 1 \mathrm{H}_{2} \mathrm{O} / \mathrm{ACN}\right)$ of $\mathrm{NRH}$ triacetate (7). & 16 \\
\hline Fig. 17S HRMS $\left(1: 1 \mathrm{H}_{2} \mathrm{O} / \mathrm{ACN}\right)$ of NRH triacetate (7). & 17 \\
\hline Fig. 18S ${ }^{1} \mathrm{H}$ NMR $\left(\mathrm{D}_{2} \mathrm{O}\right)$ of NRH $(1)$. & 18 \\
\hline Fig. 19S ${ }^{13} \mathrm{C}$ NMR $\left(\mathrm{D}_{2} \mathrm{O}\right)$ of $\mathrm{NRH}(\mathbf{1})$. & 19 \\
\hline Fig. $20 \mathrm{~S}^{1} \mathrm{H}-{ }^{1} \mathrm{H}$ correlation (COSY) NMR $\left(\mathrm{D}_{2} \mathrm{O}\right)$ of $\mathrm{NRH}(\mathbf{1})$. & 20 \\
\hline Fig. 21S ${ }^{1} \mathrm{H}-{ }^{13} \mathrm{C}$ correlation (HSQC) NMR $\left(\mathrm{D}_{2} \mathrm{O}\right)$ of $\mathrm{NRH}(\mathbf{1})$. & 21 \\
\hline Fig. 22S MS $\left(1: 1 \mathrm{H}_{2} \mathrm{O} / \mathrm{ACN}\right)$ of $\mathrm{NRH}(\mathbf{1})$. & 22 \\
\hline Fig. 23S HRMS $\left(1: 1 \mathrm{H}_{2} \mathrm{O} / \mathrm{ACN}\right)$ of of $\mathrm{NRH}(\mathbf{1})$. & 23 \\
\hline Fig. 24S ${ }^{1} \mathrm{H}$ NMR (acetone- $d_{6}$ ) of crude mixture of 1,2- $(\mathbf{8}), 1,4-(7)$, and 1,6-NRH TA (9) (reduction in $\mathrm{H}_{2} \mathrm{O} / \mathrm{DCM}$ ). & 24 \\
\hline $\begin{array}{l}\text { Fig. 25S Data on MPLC (Teledyne) chromatographic purification of crude mixture of 1,2- (8), 1,4- (7), and 1,6-NRH TA } \\
\left.\text { (9) (reduction in } \mathrm{H}_{2} \mathrm{O} / \mathrm{DCM}\right) \text {. }\end{array}$ & 25 \\
\hline Fig. 26S ${ }^{1} \mathrm{H}$ NMR (acetone- $d_{6}$ ) of 1,2-NRH TA (8) (portion A) (reduction in $\mathrm{H}_{2} \mathrm{O} / \mathrm{DCM}$ ). & 26 \\
\hline Fig. 27S ${ }^{13} \mathrm{C}$ NMR (acetone- $d_{6}$ ) of 1,2-NRH TA (8) (portion A) (reduction in $\mathrm{H}_{2} \mathrm{O} / \mathrm{DCM}$ ). & 27 \\
\hline Fig. 28S MS $\left(1: 1 \mathrm{H}_{2} \mathrm{O} / \mathrm{ACN}\right)$ of 1,2-NRH TA (8) & 28 \\
\hline Fig. 29S HRMS $\left(1: 1 \mathrm{H}_{2} \mathrm{O} / \mathrm{ACN}\right)$ of 1,2-NRH TA (8): full scan. & 29 \\
\hline Fig. 30S HRMS $\left(1: 1 \mathrm{H}_{2} \mathrm{O} / \mathrm{ACN}\right)$ of 1,2-NRH TA $(\mathbf{8})$ : parent peak. & 30 \\
\hline Fig. 31S HRMS $\left(1: 1 \mathrm{H}_{2} \mathrm{O} / \mathrm{ACN}\right)$ of 1,2-NRH TA (8): fragmentation of parent peak. & 31 \\
\hline Fig. 32S ${ }^{1} \mathrm{H}$ NMR (acetone- $d_{6}$ ) of 1,4- (7) and 1,6-NRH TA (9) mixture (portion $\mathrm{B}$ ) (reduction in $\mathrm{H}_{2} \mathrm{O} / \mathrm{DCM}$ ). & 32 \\
\hline $\begin{array}{l}\text { Fig. 33S }{ }^{1} \mathrm{H} \text { NMR (acetone- } d_{6} \text { ) of 1,6-NRH TA (9) containing admixture of 1,4-NRH TA (7) (18-20 mol\%) (portion C) } \\
\left.\text { (reduction in } \mathrm{H}_{2} \mathrm{O} / \mathrm{DCM}\right) \text {. }\end{array}$ & 33 \\
\hline $\begin{array}{l}\text { Fig. 34S }{ }^{13} \mathrm{C} \text { NMR (acetone- } d_{6} \text { ) of 1,6-NRH TA (9) containing admixture of 1,4-NRH TA (7) (18-20 mol\%) (portion C) } \\
\left.\text { (reduction in } \mathrm{H}_{2} \mathrm{O} / \mathrm{DCM}\right) \text {. }\end{array}$ & 34 \\
\hline Fig. 35S MS (1:1 $\left.\mathrm{H}_{2} \mathrm{O} / \mathrm{ACN}\right)$ 1,6-NRH TA (9) & 35 \\
\hline Fig. 36S HRMS (1:1 H2O/ACN) of 1,6-NRH TA (9): full scan. & 36 \\
\hline
\end{tabular}


Fig. 37S HRMS $\left(1: 1 \mathrm{H}_{2} \mathrm{O} / \mathrm{ACN}\right)$ of 1,6-NRH TA (9): parent peak.

Fig. 38S HRMS (1:1 $\left.\mathrm{H}_{2} \mathrm{O} / \mathrm{ACN}\right)$ of 1,6-NRH TA (9): fragmentation of parent peak.

Fig. 39S Comparison of fragmentation of 1,2-NRH TA (8) (sample mm308a) and 1,6-NRH TA (9) (sample mm308b).

Fig. 40S ${ }^{1}$ H NMR (acetone- $d_{6}$ ) of crude mixture of 1,2- $(\mathbf{8}), 1,4-(7)$, and 1,6-NRH TA (9) (reduction in DMF).

Fig. $41 \mathrm{~S}$ Side-by-side comparison of ${ }^{1} \mathrm{H}$ NMRs (acetone- $d_{6}$ ) of portions C-F containing 1,6-NRH TA (9) and variable amounts of 1,4-NRH TA (7).

Fig. 42S ${ }^{1} \mathrm{H}$ NMR (acetone- $d_{6}$ ) of 1,2-NRH TA (8).

Fig. 43S ${ }^{13} \mathrm{C}$ NMR (acetone- $\left.d_{6}\right)$ of 1,2-NRH TA (8).

Fig. 44S ${ }^{1} \mathrm{H}^{1}{ }^{\mathrm{H}}$ correlation (COSY) NMR (acetone- $d_{6}$ ) of 1,2-NRH TA (8).

Fig. $45 \mathrm{~S}^{1} \mathrm{H}^{-13} \mathrm{C}$ correlation (HSQC) NMR (acetone- $d_{6}$ ) of 1,2-NRH TA $(8)$.

Fig. 46S ${ }^{1} \mathrm{H}$ full NMR(acetone- $d_{6}$ ) spectrum of 1,6-NRH TA (9).

Fig. 47S ${ }^{1} \mathrm{H}$ zoomed NMR spectrum (acetone- $d_{6}$ ) of 1,6-NRH TA (9).

Fig. 48S ${ }^{13} \mathrm{C}$ NMR (acetone- $d_{6}$ ) of 1,6-NRH TA (9).

Fig. 49S ${ }^{1} \mathrm{H}^{-1} \mathrm{H}$ correlation (COSY) NMR (acetone- $d_{6}$ ) of 1,6-NRH TA (9).

Fig. 50S ${ }^{1} \mathrm{H}_{-}{ }^{13} \mathrm{C}$ correlation (HSQC) NMR (acetone- $d_{6}$ ) of 1,6-NRH TA (9).

Fig. 51S ${ }^{1} \mathrm{H}$ full NMR $\left(\mathrm{D}_{2} \mathrm{O}\right)$ spectrum of 1,2-NRH (2).

Fig. 52S ${ }^{1} \mathrm{H}$ zoomed NMR $\left(\mathrm{D}_{2} \mathrm{O}\right)$ spectrum of 1,2-NRH (2).

Fig. 53S ${ }^{13} \mathrm{C} \mathrm{NMR}\left(\mathrm{D}_{2} \mathrm{O}\right)$ of 1,2-NRH (2).

Fig. 54S ${ }^{1} \mathrm{H}^{-1} \mathrm{H}$ correlation (COSY) NMR $\left(\mathrm{D}_{2} \mathrm{O}\right)$ of 1,2-NRH (2).

Fig. 55S ${ }^{1} \mathrm{H}^{13} \mathrm{C}$ correlation (HSQC) NMR $\left(\mathrm{D}_{2} \mathrm{O}\right)$ of 1,2-NRH (2).

Fig. 56S HRMS $\left(1: 1 \mathrm{H}_{2} \mathrm{O} / \mathrm{ACN}\right)$ of 1,2-NRH (2): full scan.

Fig. 57S HRMS (1:1 $\left.\mathrm{H}_{2} \mathrm{O} / \mathrm{ACN}\right)$ of 1,2-NRH (2): parent peak.

Fig. 58S HRMS $\left(1: 1 \mathrm{H}_{2} \mathrm{O} / \mathrm{ACN}\right)$ of 1,2-NRH (2): fragmentation of parent peak.

Fig. 59S ${ }^{1} \mathrm{H}$ full NMR $\left(\mathrm{D}_{2} \mathrm{O}\right)$ spectrum of 1,6-NRH (3).

Fig. 60S ${ }^{1} \mathrm{H}$ zoomed NMR $\left(\mathrm{D}_{2} \mathrm{O}\right)$ spectrum of 1,6-NRH (3).

Fig. 61S ${ }^{13} \mathrm{C}$ NMR $\left(\mathrm{D}_{2} \mathrm{O}\right)$ of 1,6-NRH (3).

Fig. 62S ${ }^{1} \mathrm{H}-{ }^{1} \mathrm{H}$ correlation (COSY) NMR $\left(\mathrm{D}_{2} \mathrm{O}\right)$ of $1,6-\mathrm{NRH}(3)$.

Fig. 63S ${ }^{1} \mathrm{H}_{-}{ }^{13} \mathrm{C}$ correlation (HSQC) NMR $\left(\mathrm{D}_{2} \mathrm{O}\right)$ of $1,6-\mathrm{NRH}(3)$.

Fig. 64S HRMS $\left(1: 1 \mathrm{H}_{2} \mathrm{O} / \mathrm{ACN}\right)$ of 1,6-NRH (3): full scan.

Fig. 65S HRMS $\left(1: 1 \mathrm{H}_{2} \mathrm{O} / \mathrm{ACN}\right)$ of 1,6-NRH (3): parent peak.

Fig. 66S HRMS $\left(1: 1 \mathrm{H}_{2} \mathrm{O} / \mathrm{ACN}\right)$ of 1,6-NRH (3): fragmentation of parent peak.

Fig. 67S Comparison of fragmentation of 1,2-NRH (2) (sample mm320) and 1,6-NRH (3) (sample mm315).

65


NMR, MS and HRMS data

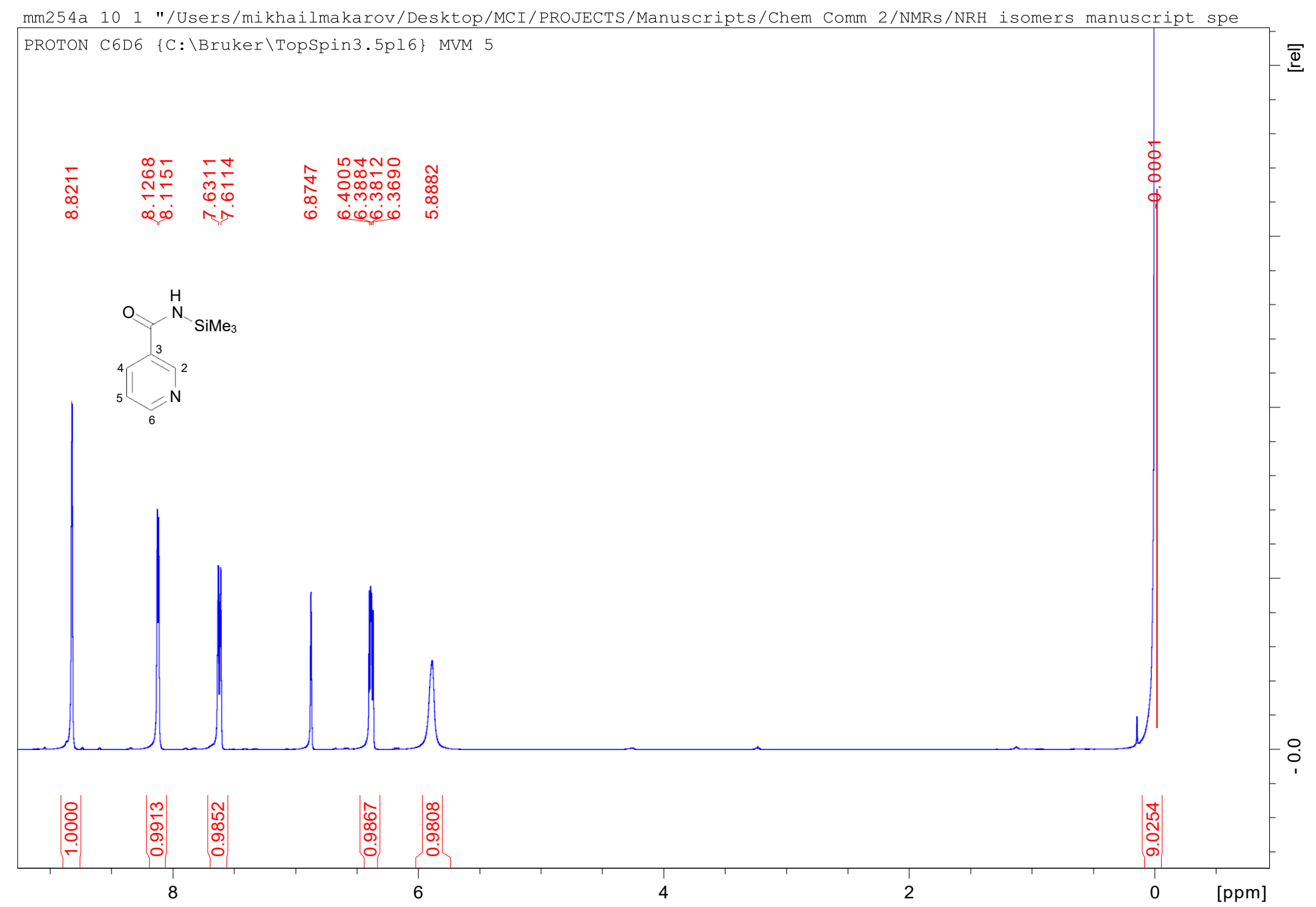

Fig. 3S ${ }^{1} \mathrm{H}$ NMR $\left(\mathrm{C}_{6} \mathrm{D}_{6}\right)$ of $N$-(trimethylsilyl)Nam. 


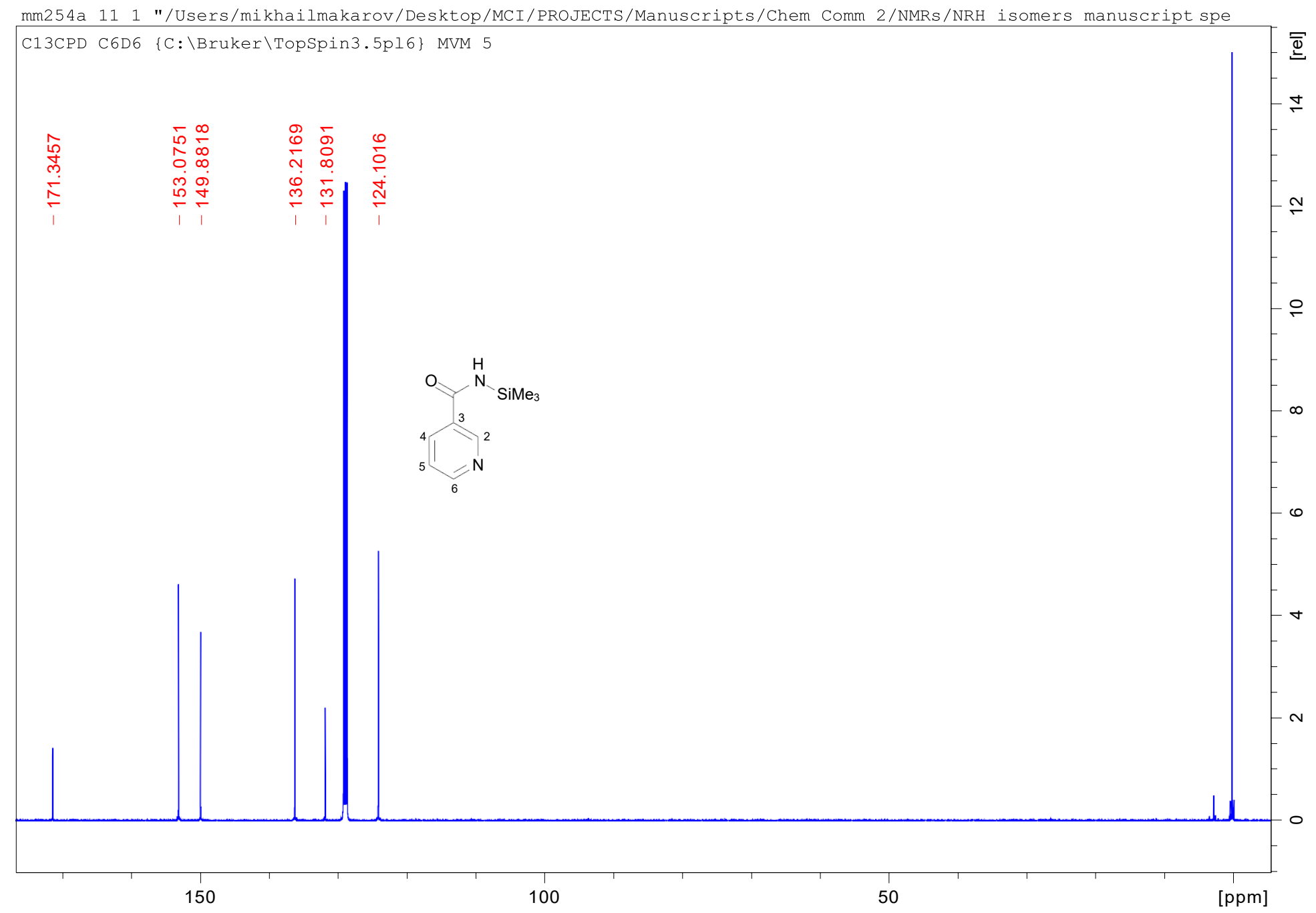

Fig. 4S ${ }^{13} \mathrm{C}$ NMR $\left(\mathrm{C}_{6} \mathrm{D}_{6}\right)$ of $N$-(trimethylsilyl)Nam. 


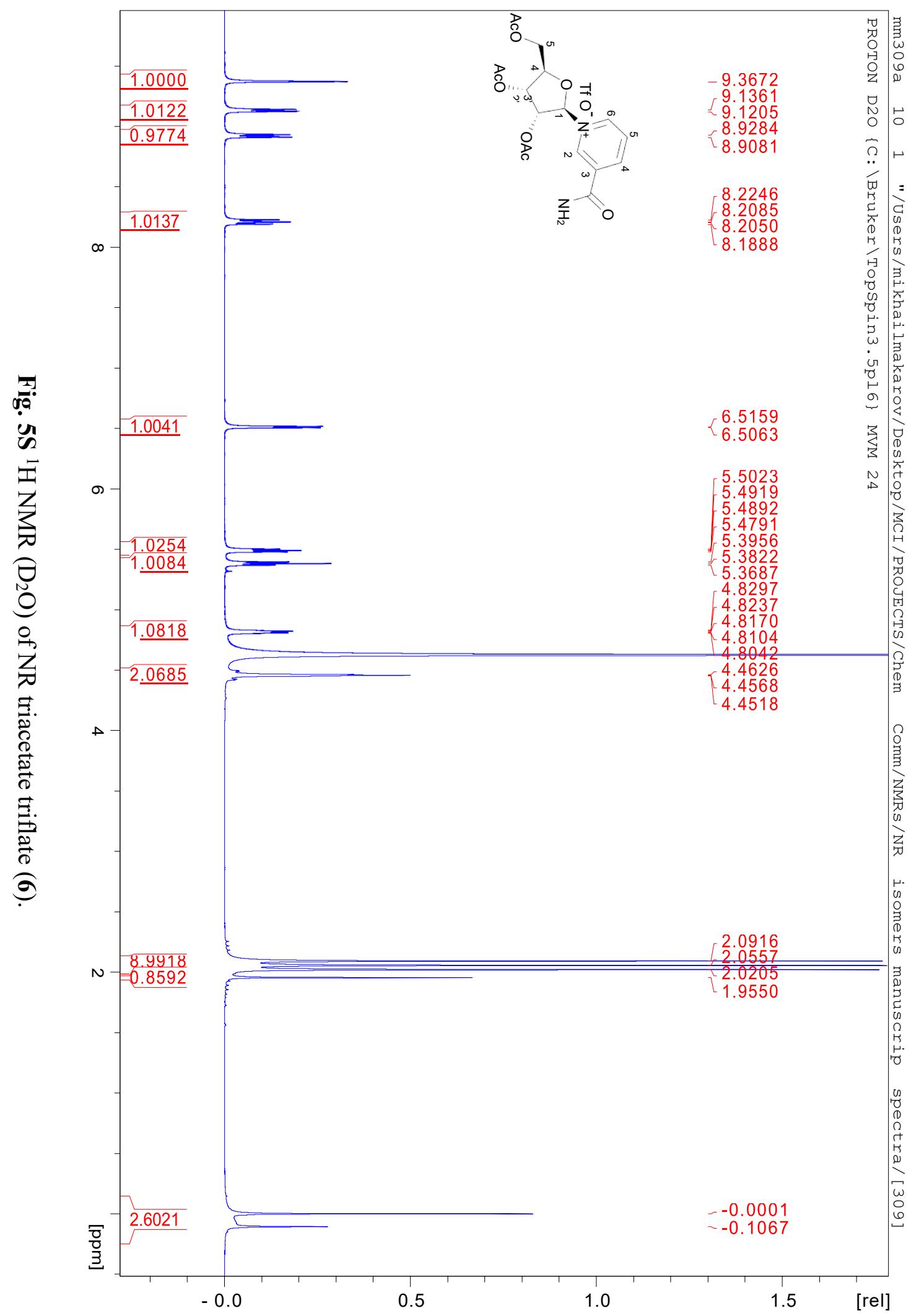


MM086a 31 "/Users/mikhailmakarov/Desktop/MCI/PROJECTS/Chem Comm/NMRs/NRH isomers manuscript spectra"

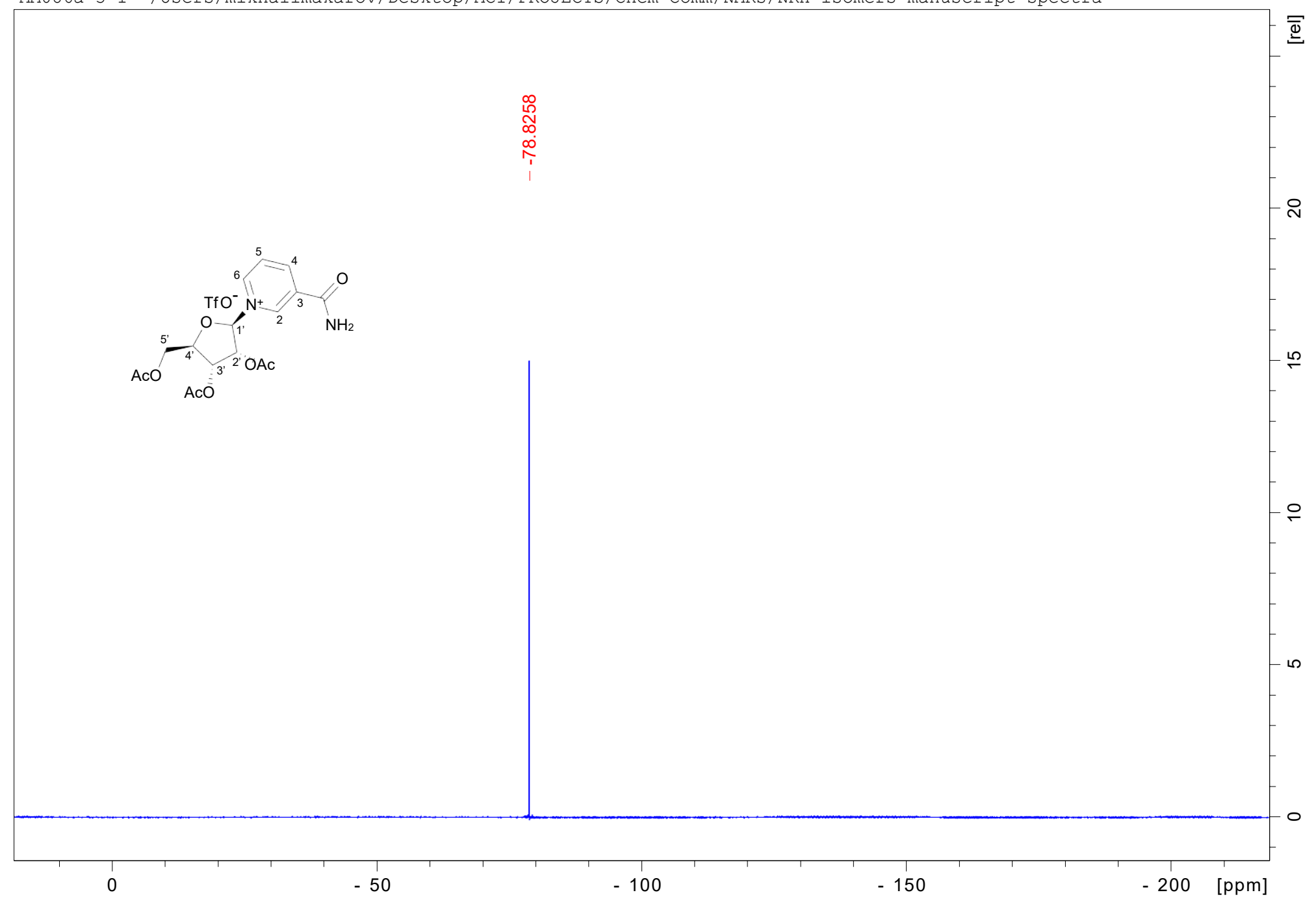

Fig. 6S ${ }^{19} \mathrm{~F}$ NMR $\left(\mathrm{D}_{2} \mathrm{O}\right)$ of NR triacetate triflate $(6)$. 


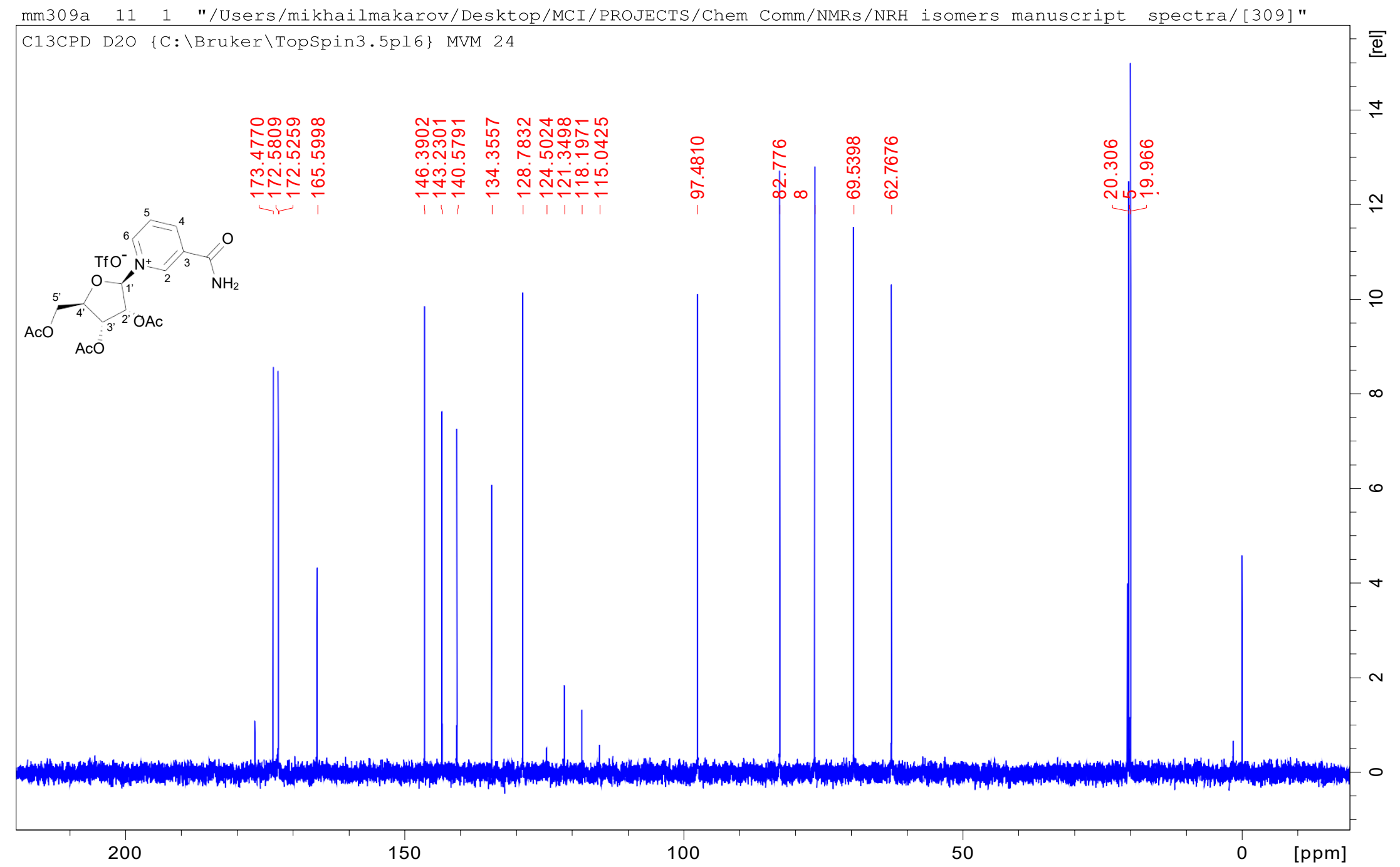

Fig. $7 \mathrm{~S}^{13} \mathrm{C}$ NMR $\left(\mathrm{D}_{2} \mathrm{O}\right)$ of NR triacetate triflate (6). 


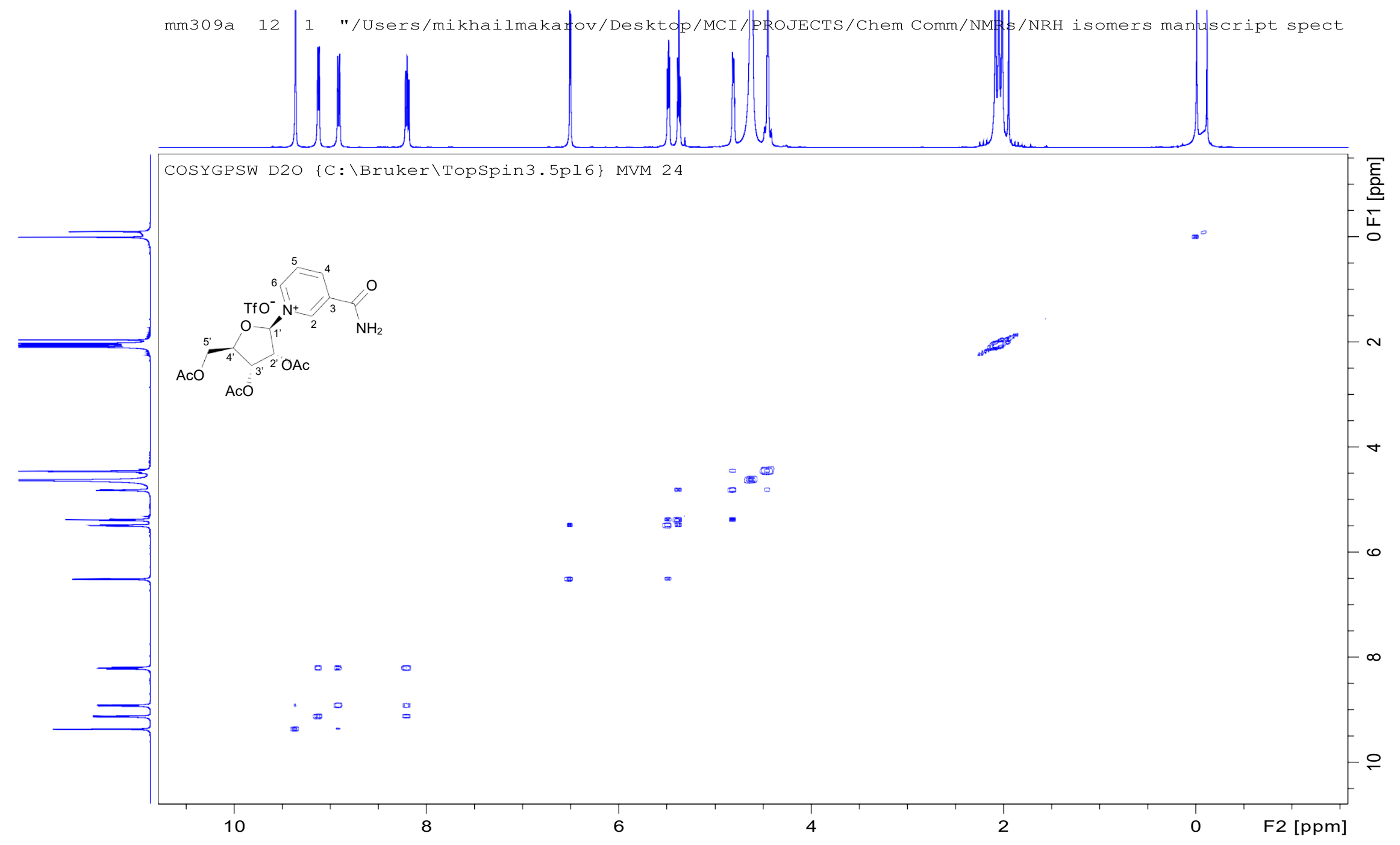

Fig. 8S ${ }^{1} \mathrm{H}-{ }^{1} \mathrm{H}$ correlation (COSY) NMR $\left(\mathrm{D}_{2} \mathrm{O}\right)$ of NR triacetate triflate (6). 


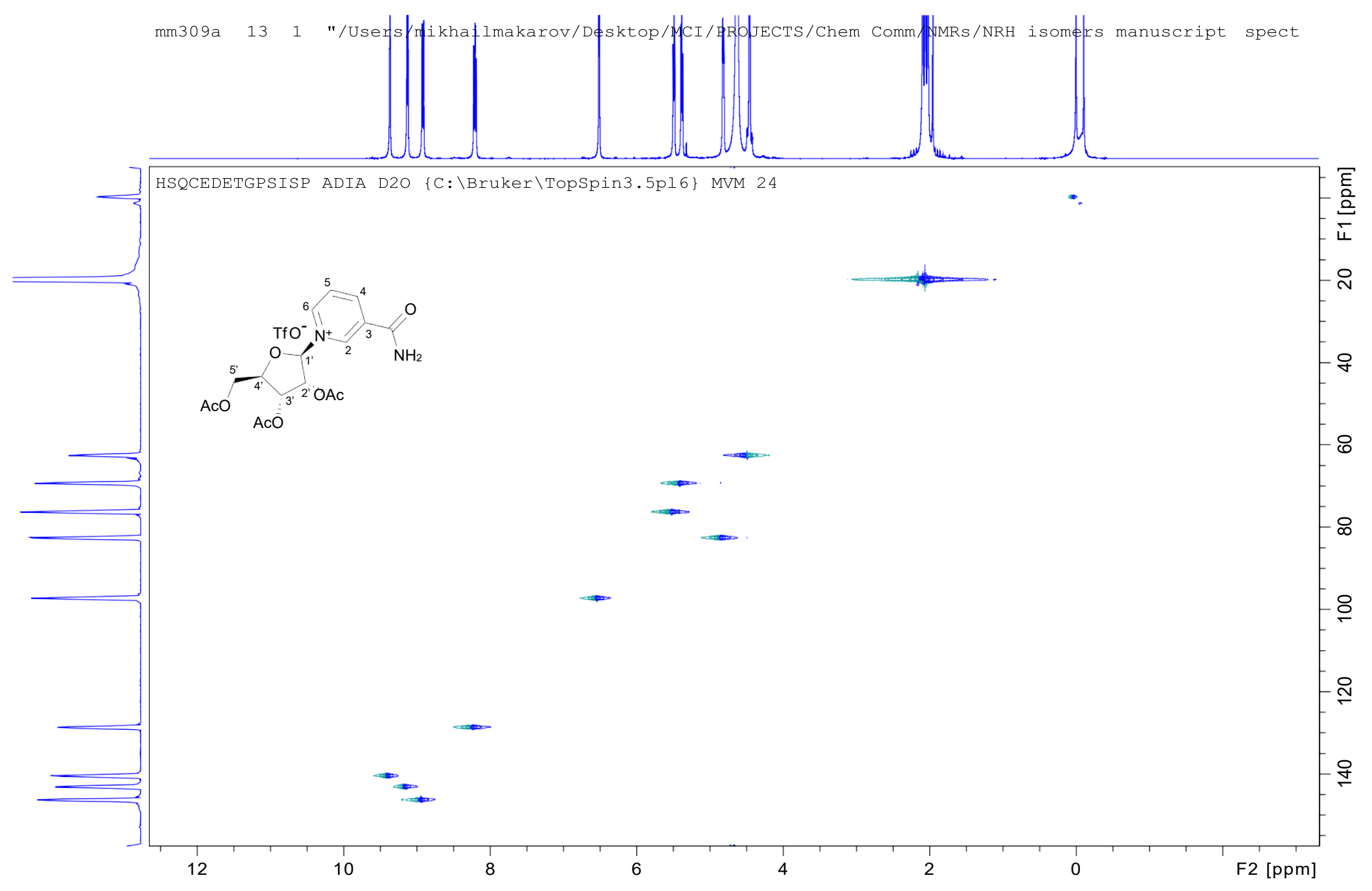

Fig. 9S ${ }^{1} \mathrm{H}-{ }^{13} \mathrm{C}$ correlation (HSQC) NMR $\left(\mathrm{D}_{2} \mathrm{O}\right)$ of NR triacetate triflate (6). 
D:IMSQ_Datalmm_072319_mm230_600mass_01

07/23/19 21:21:11

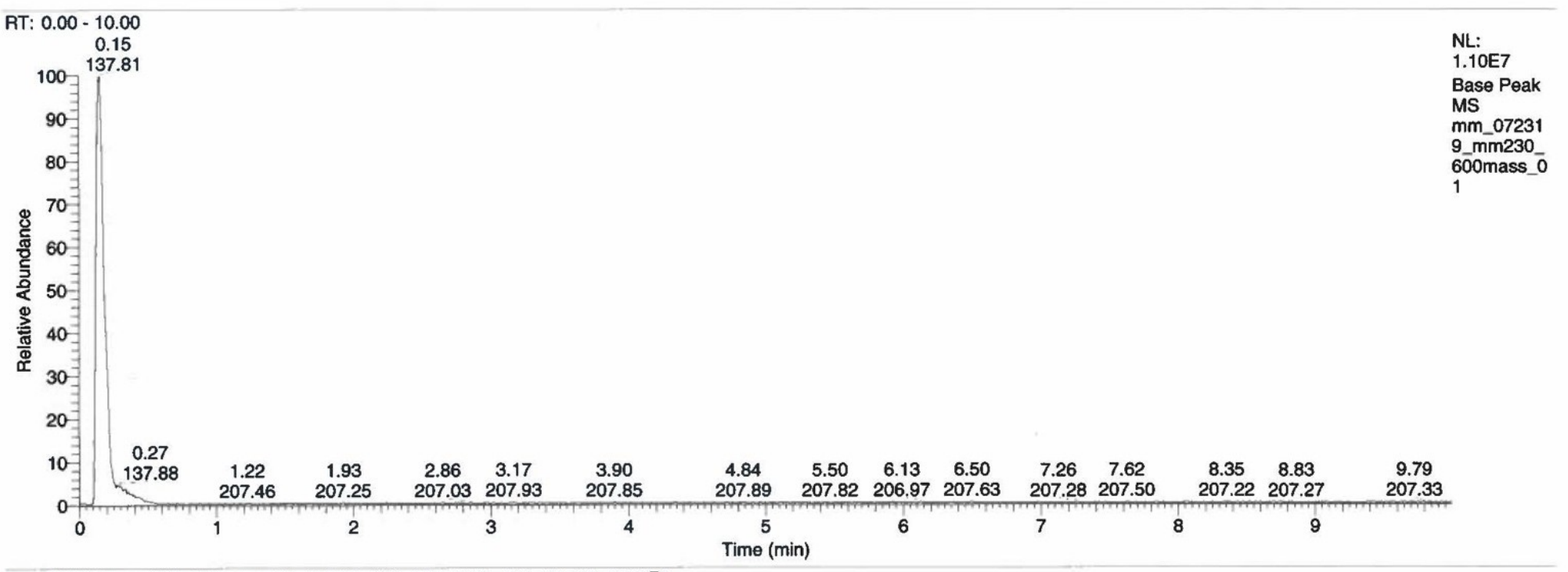

mm_072319_mm230_600mass_01 \#13-39 RT: 0.11-0.33 AV: 27 NL: 3.32E6

$\mathrm{T}:\{0,0\}+\mathrm{p}$ ESI corona sid $=75.00$ det $=918.00$ Full $\mathrm{ms}[100.00-600.00]$

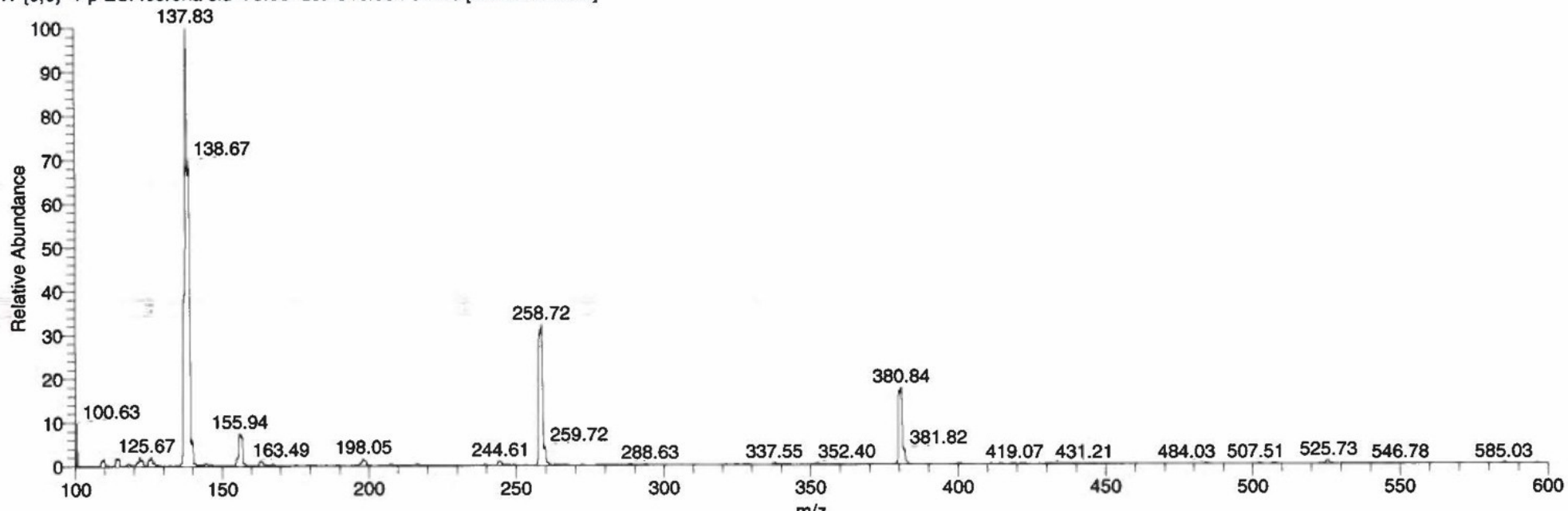

Fig. 10S MS (1:1 $\left.\mathrm{H}_{2} \mathrm{O} / \mathrm{ACN}\right)$ of NRTA triflate (6). 


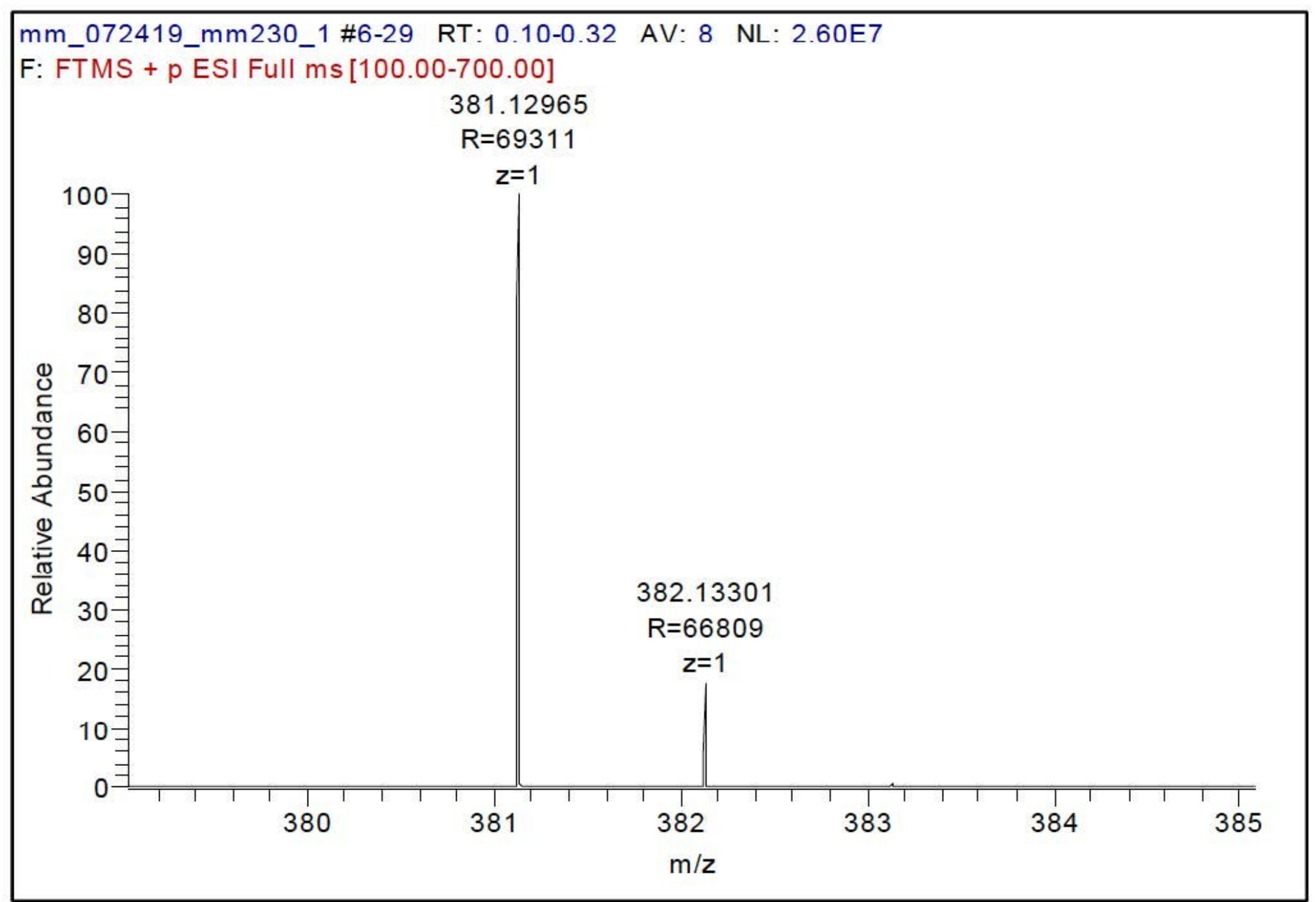

Fig. 11S HRMS (1:1 $\left.\mathrm{H}_{2} \mathrm{O} / \mathrm{ACN}\right)$ of NRTA triflate (3). 


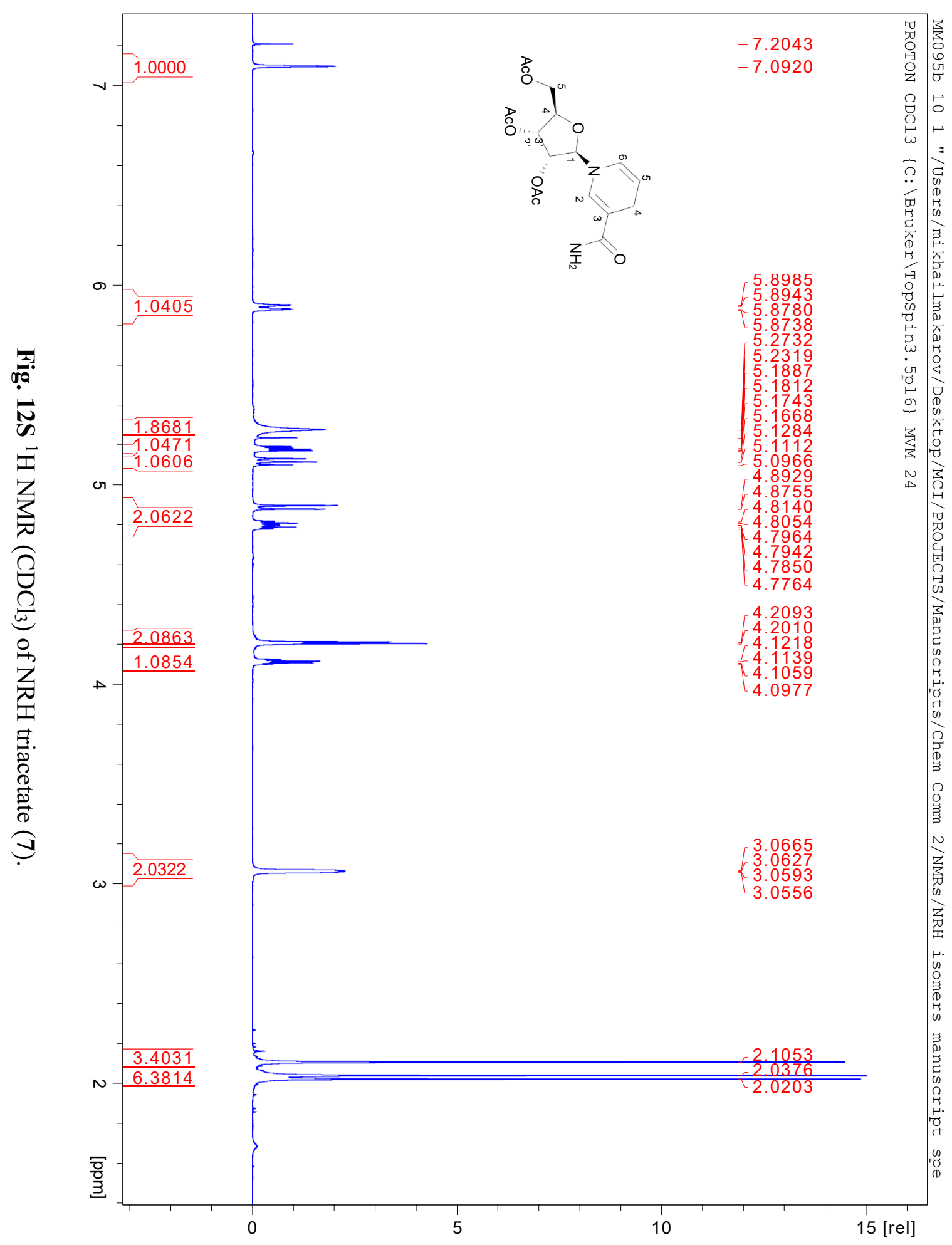




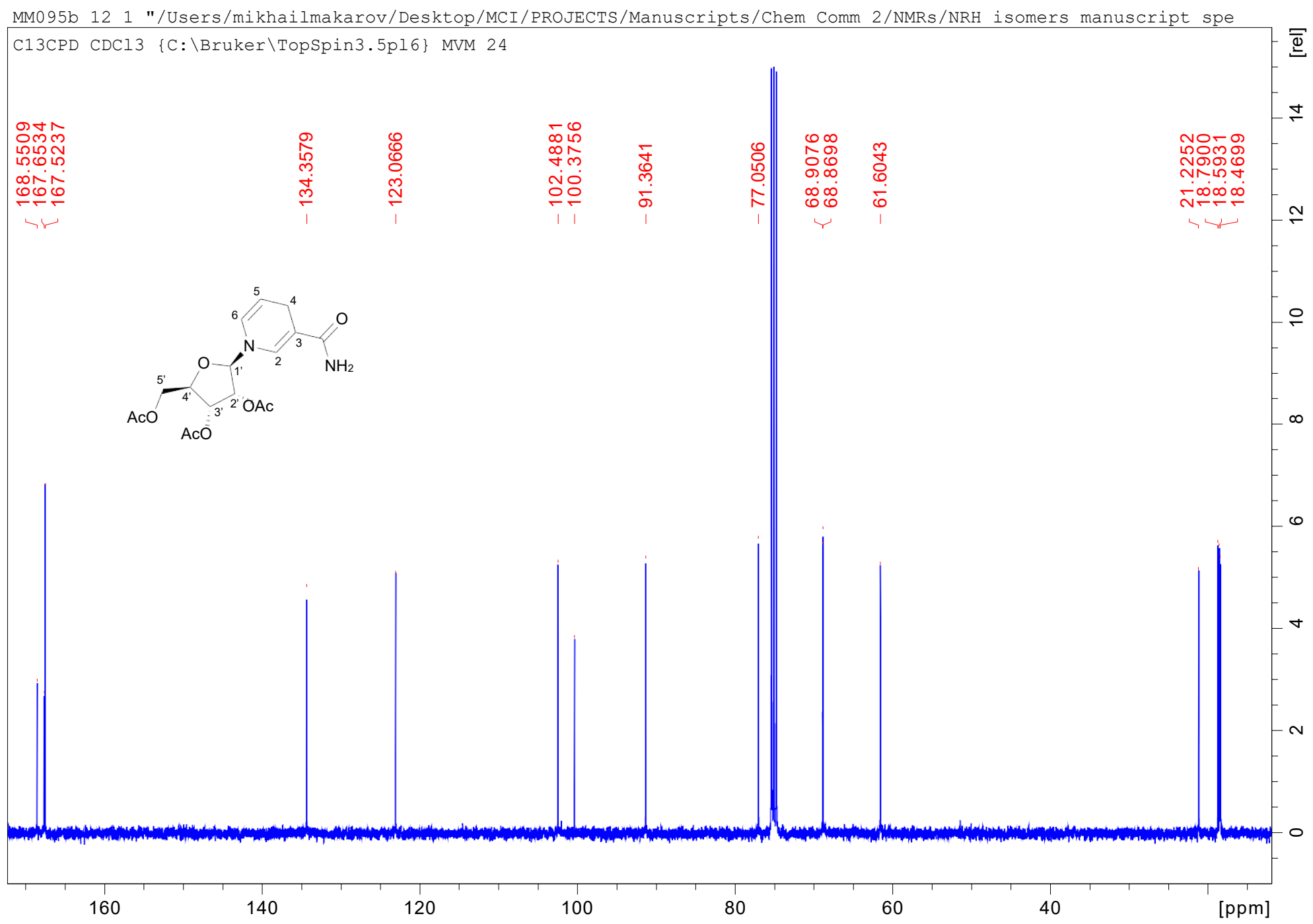

Fig. 13S ${ }^{13} \mathrm{C} \mathrm{NMR}\left(\mathrm{CDCl}_{3}\right)$ of $\mathrm{NRH}$ triacetate (7). 


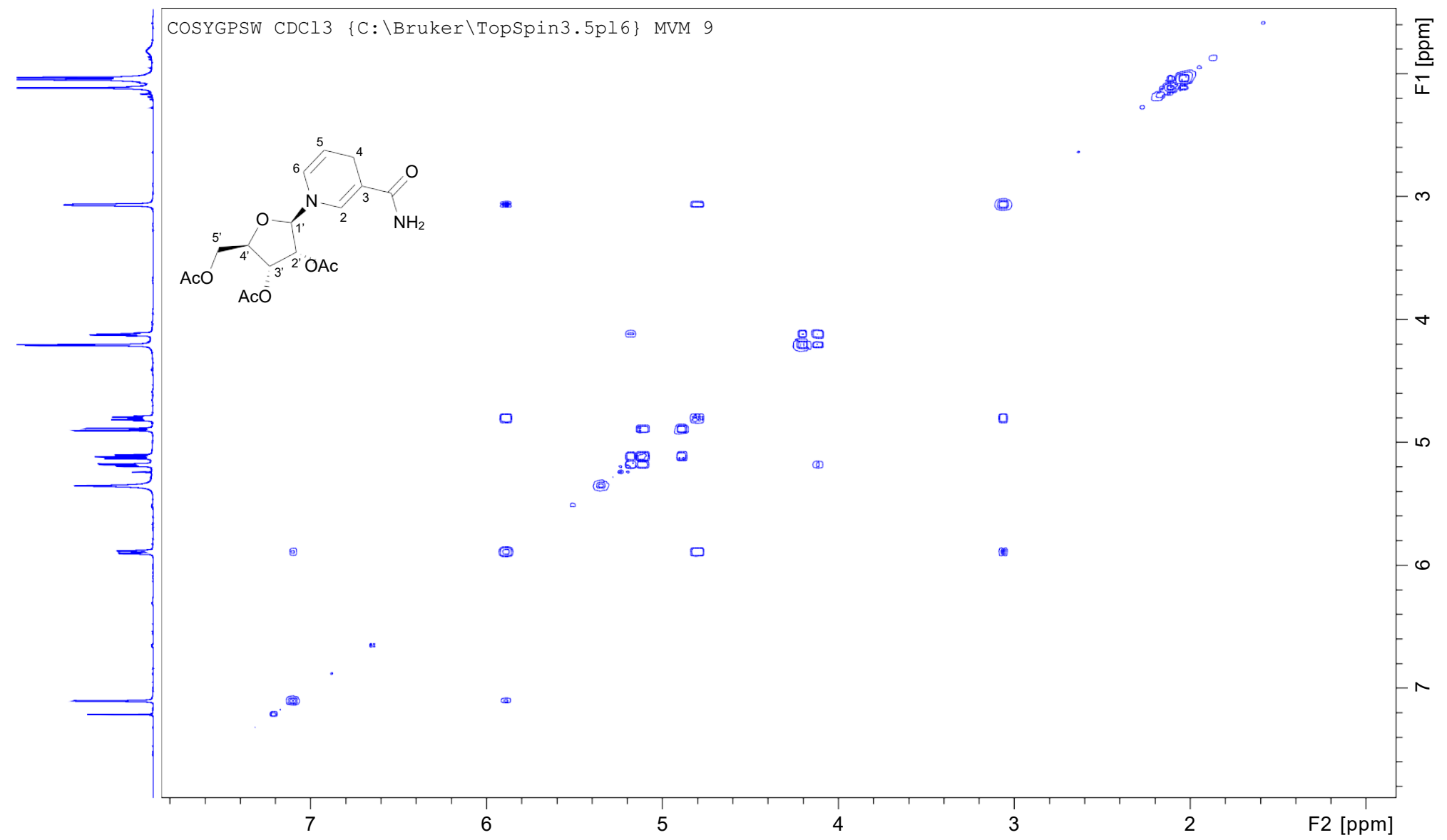

Fig. $14 \mathrm{~S}^{1} \mathrm{H}-{ }^{1} \mathrm{H}$ correlation (COSY) NMR $\left(\mathrm{CDCl}_{3}\right)$ of $\mathrm{NRH}$ triacetate (7). 


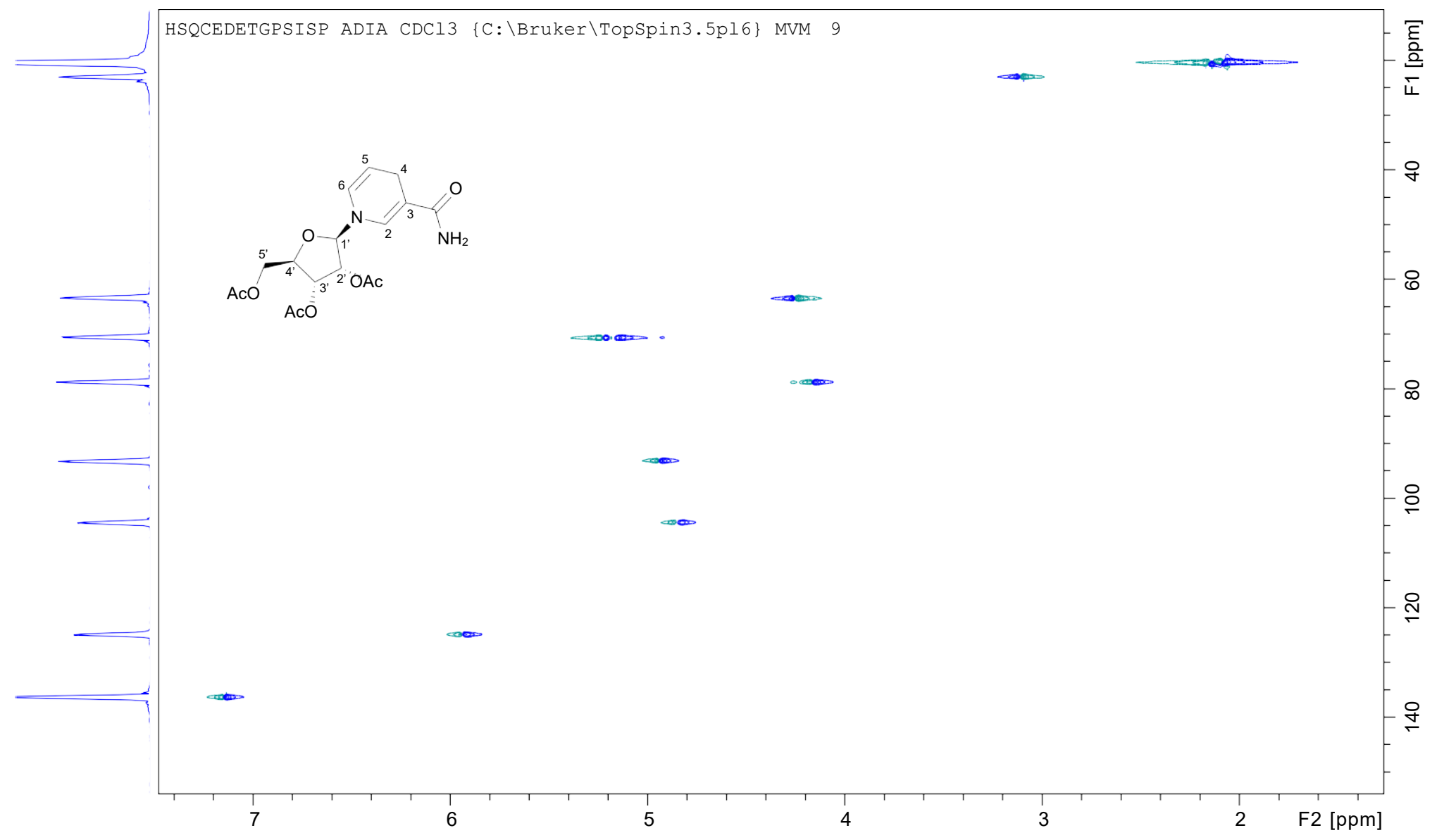

Fig. 15S ${ }^{1} \mathrm{H}_{-}{ }^{13} \mathrm{C}$ correlation (HSQC) NMR $\left(\mathrm{CDCl}_{3}\right)$ of $\mathrm{NRH}$ triacetate (7). 


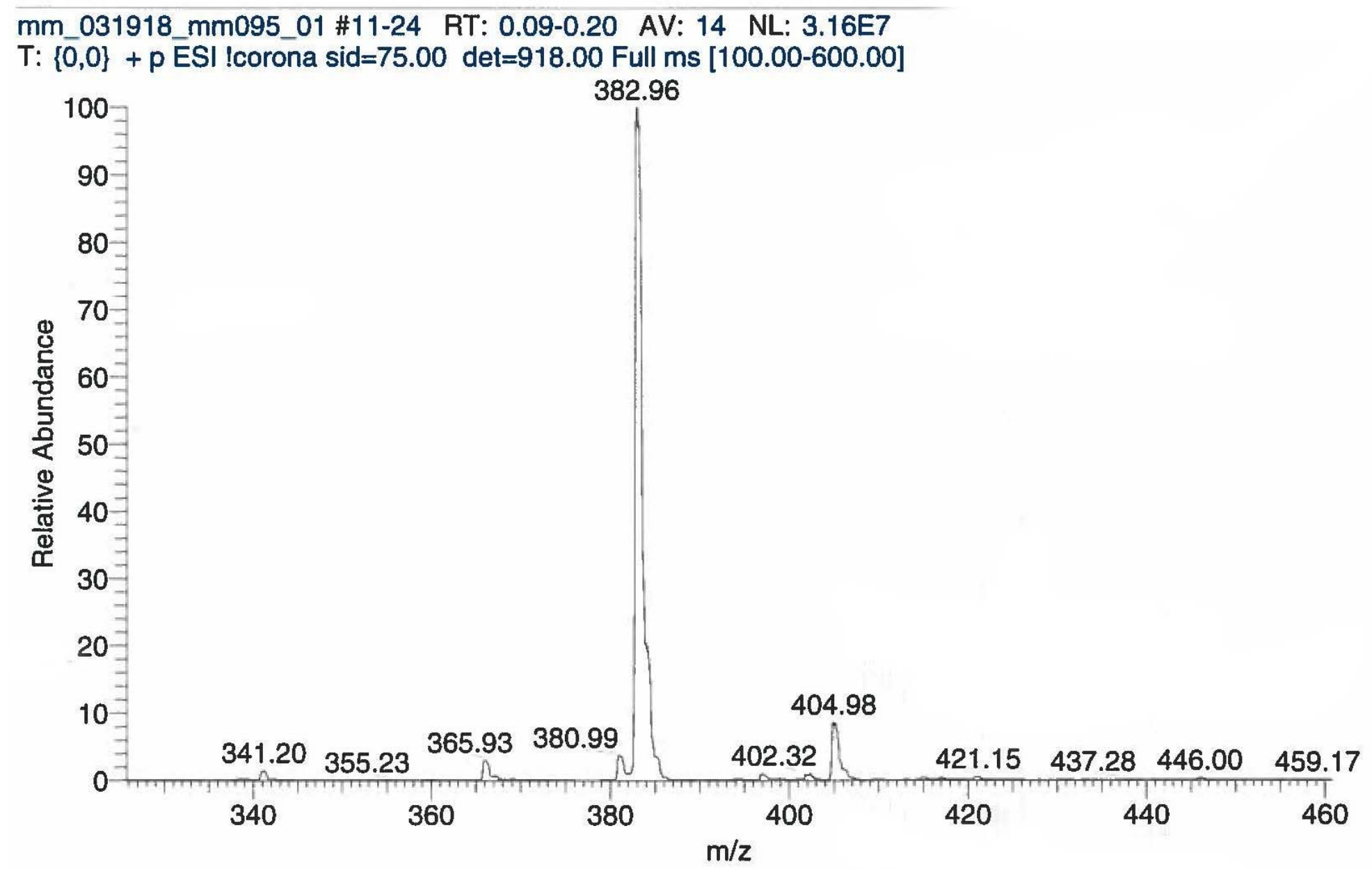

Fig. 16S MS $\left(1: 1 \mathrm{H}_{2} \mathrm{O} / \mathrm{ACN}\right)$ of $\mathrm{NRH}$ triacetate (7). 


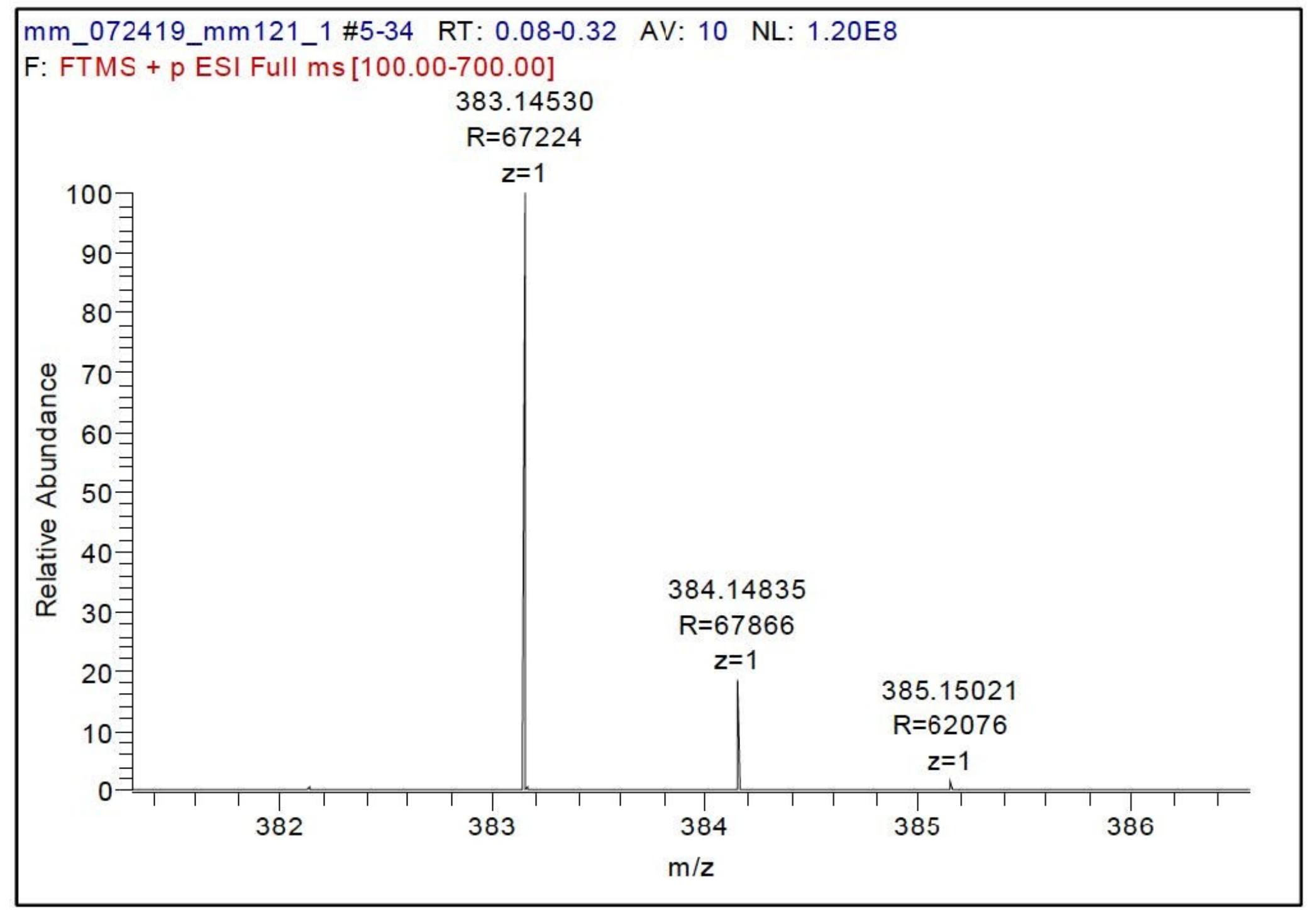

Fig. 17S HRMS $\left(1: 1 \mathrm{H}_{2} \mathrm{O} / \mathrm{ACN}\right)$ of $\mathrm{NRH}$ triacetate (7). 


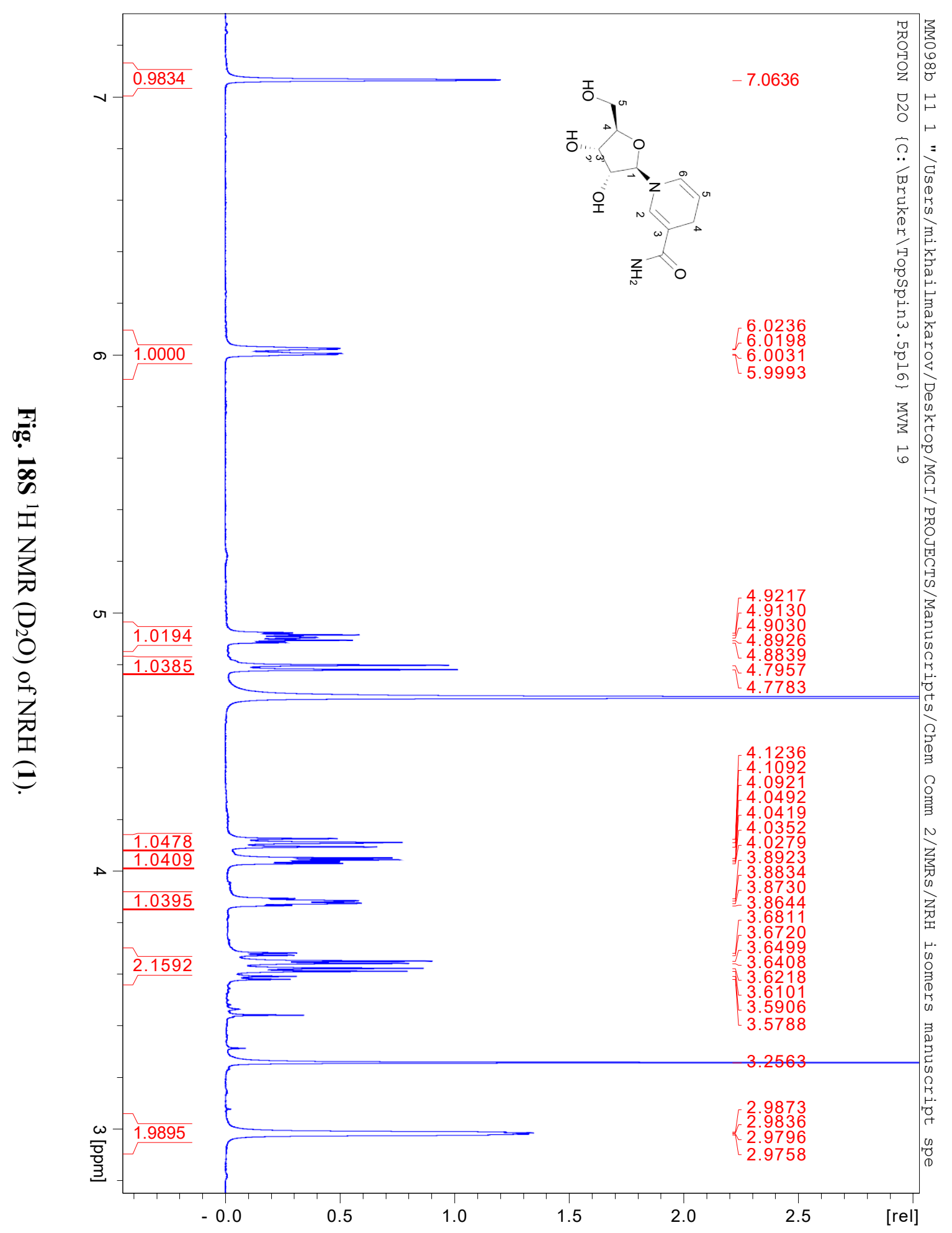




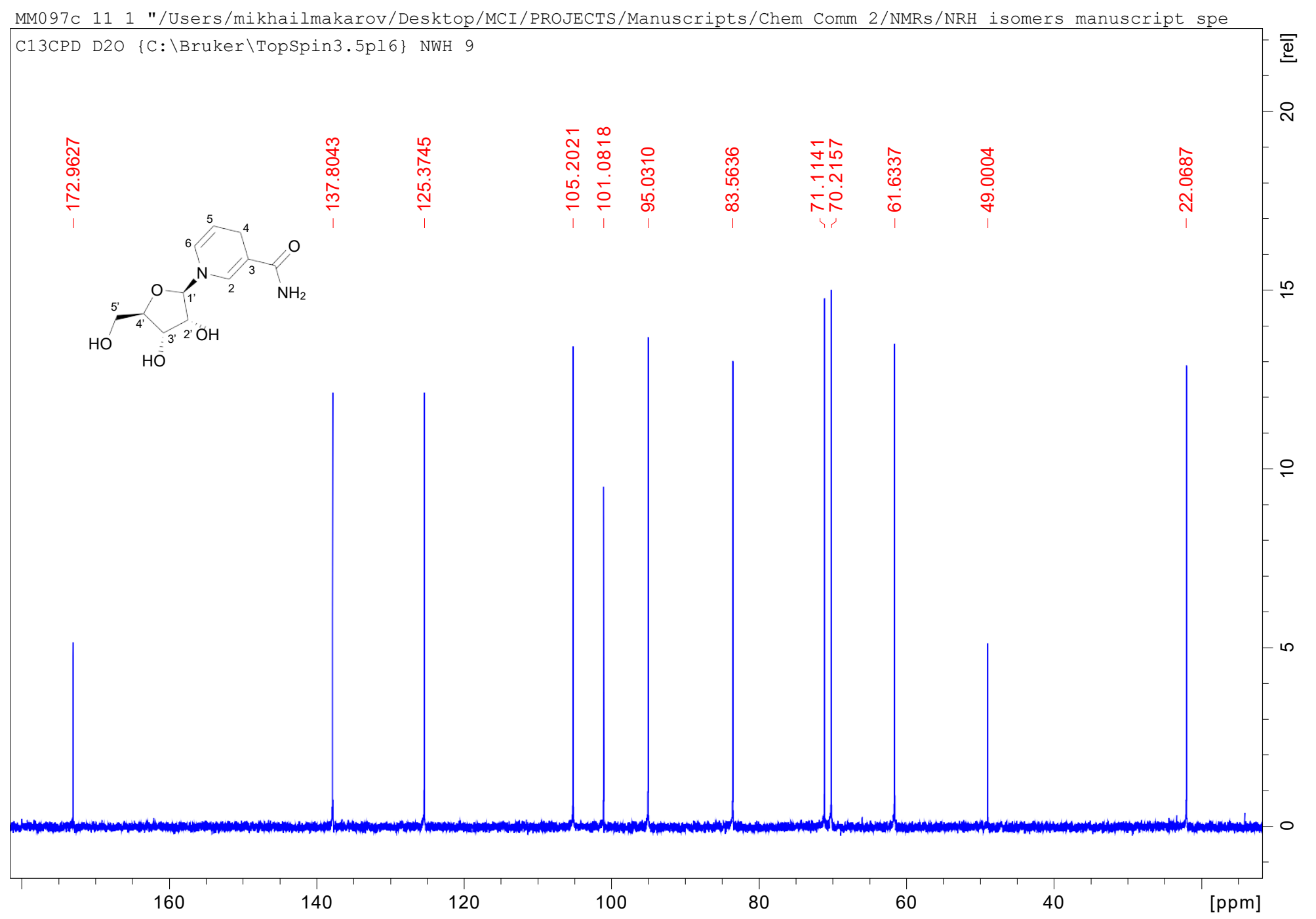

Fig. 19S ${ }^{13} \mathrm{C}$ NMR $\left(\mathrm{D}_{2} \mathrm{O}\right)$ of $\mathrm{NRH}(\mathbf{1})$. 


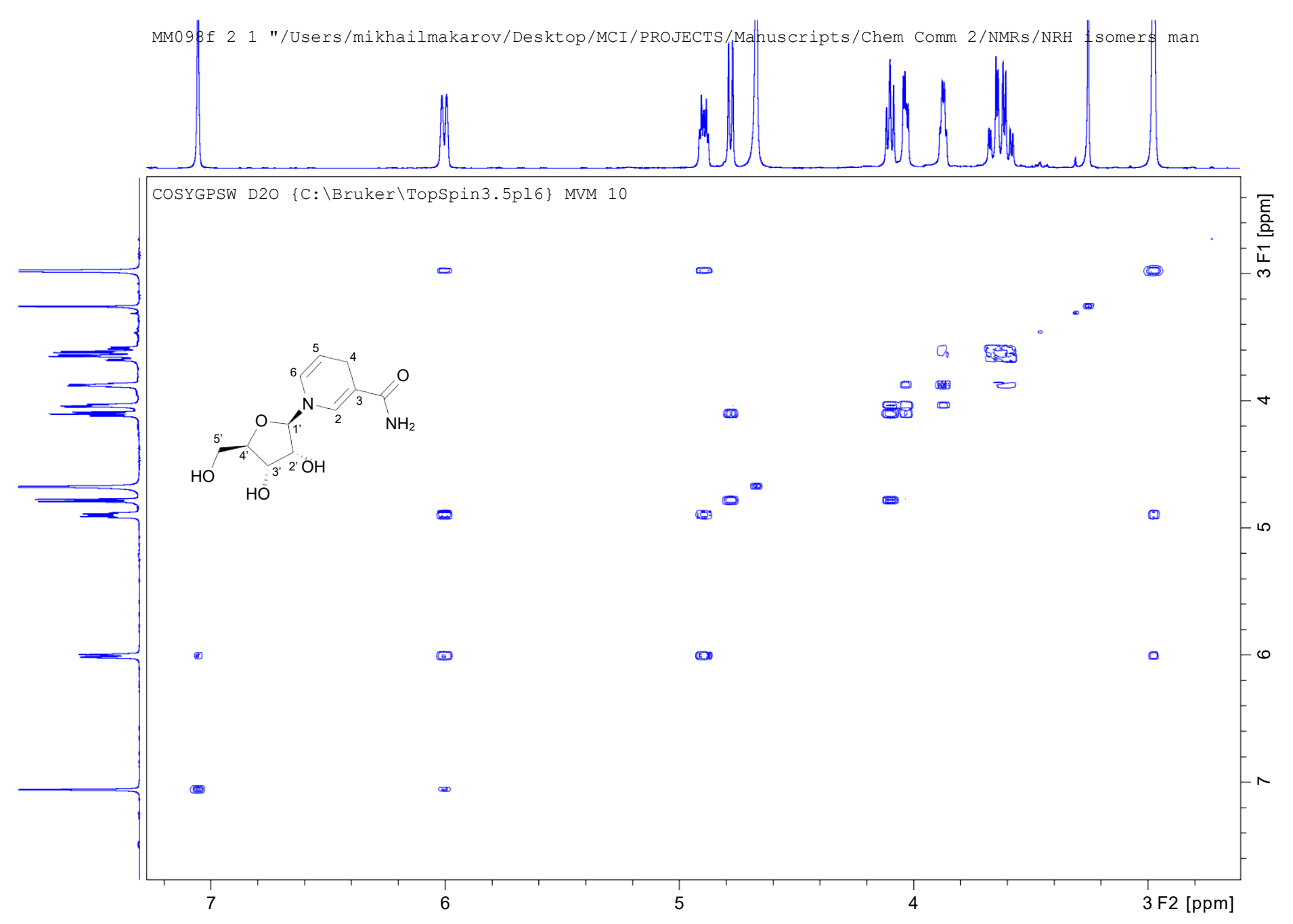

Fig. 20S ${ }^{1} \mathrm{H}-{ }^{1} \mathrm{H}$ correlation (COSY) NMR $\left(\mathrm{D}_{2} \mathrm{O}\right)$ of $\mathrm{NRH}(\mathbf{1})$. 


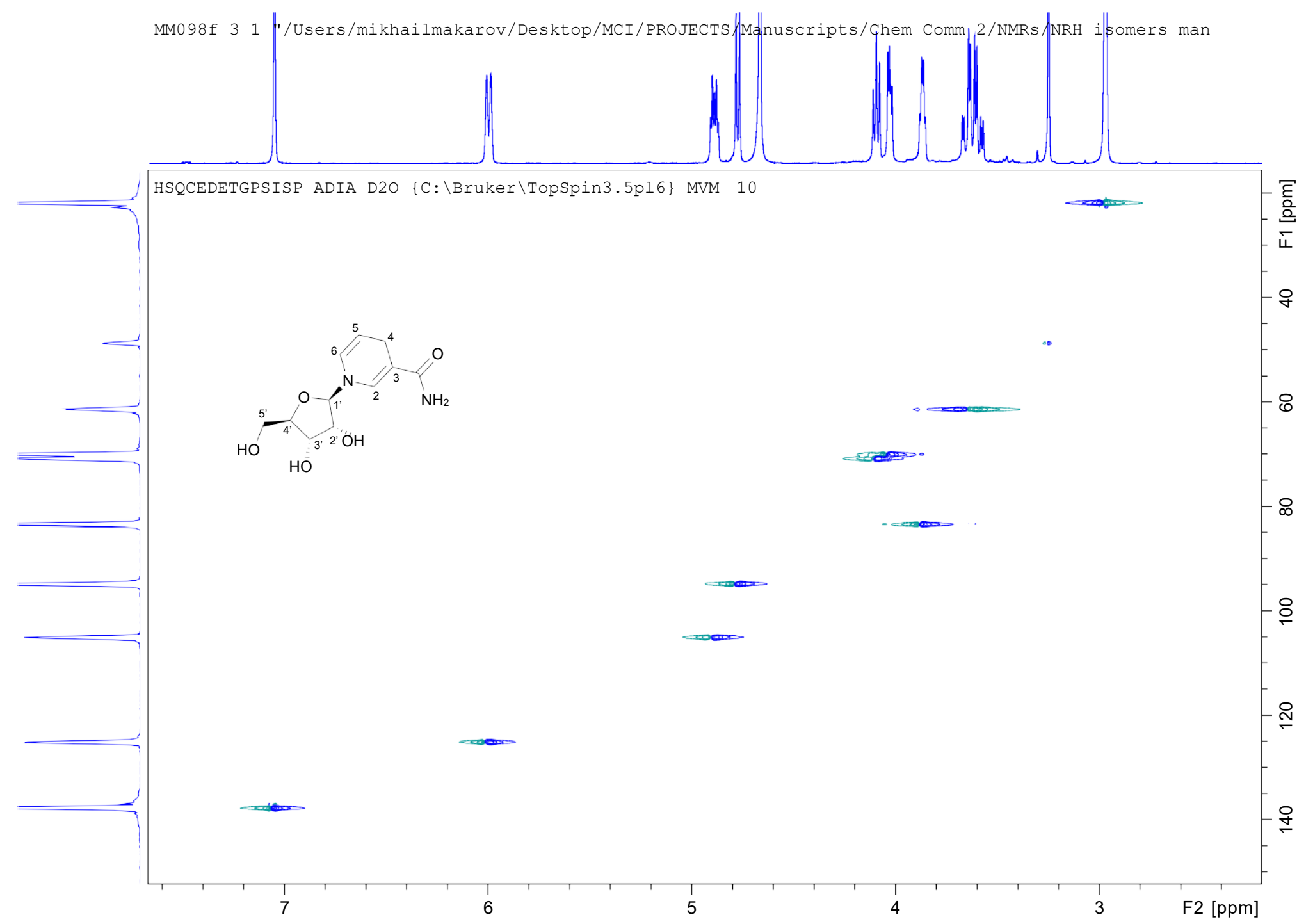

Fig. 21S ${ }^{1} \mathrm{H}^{-13} \mathrm{C}$ correlation (HSQC) NMR $\left(\mathrm{D}_{2} \mathrm{O}\right)$ of $\mathrm{NRH}(\mathbf{1})$. 


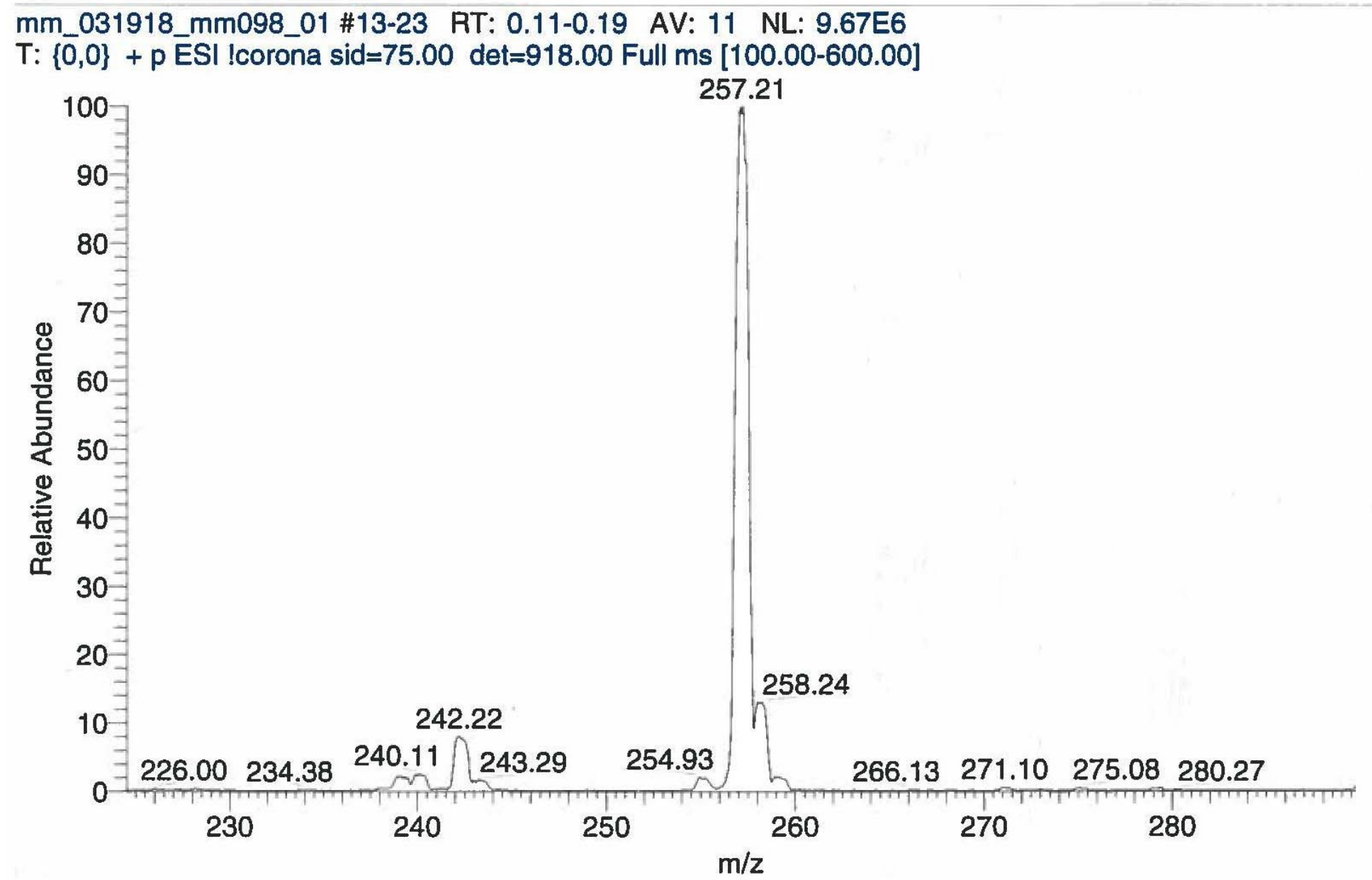

Fig. 22S MS (1:1 $\left.\mathrm{H}_{2} \mathrm{O} / \mathrm{ACN}\right)$ of $\mathrm{NRH}$ (1). 


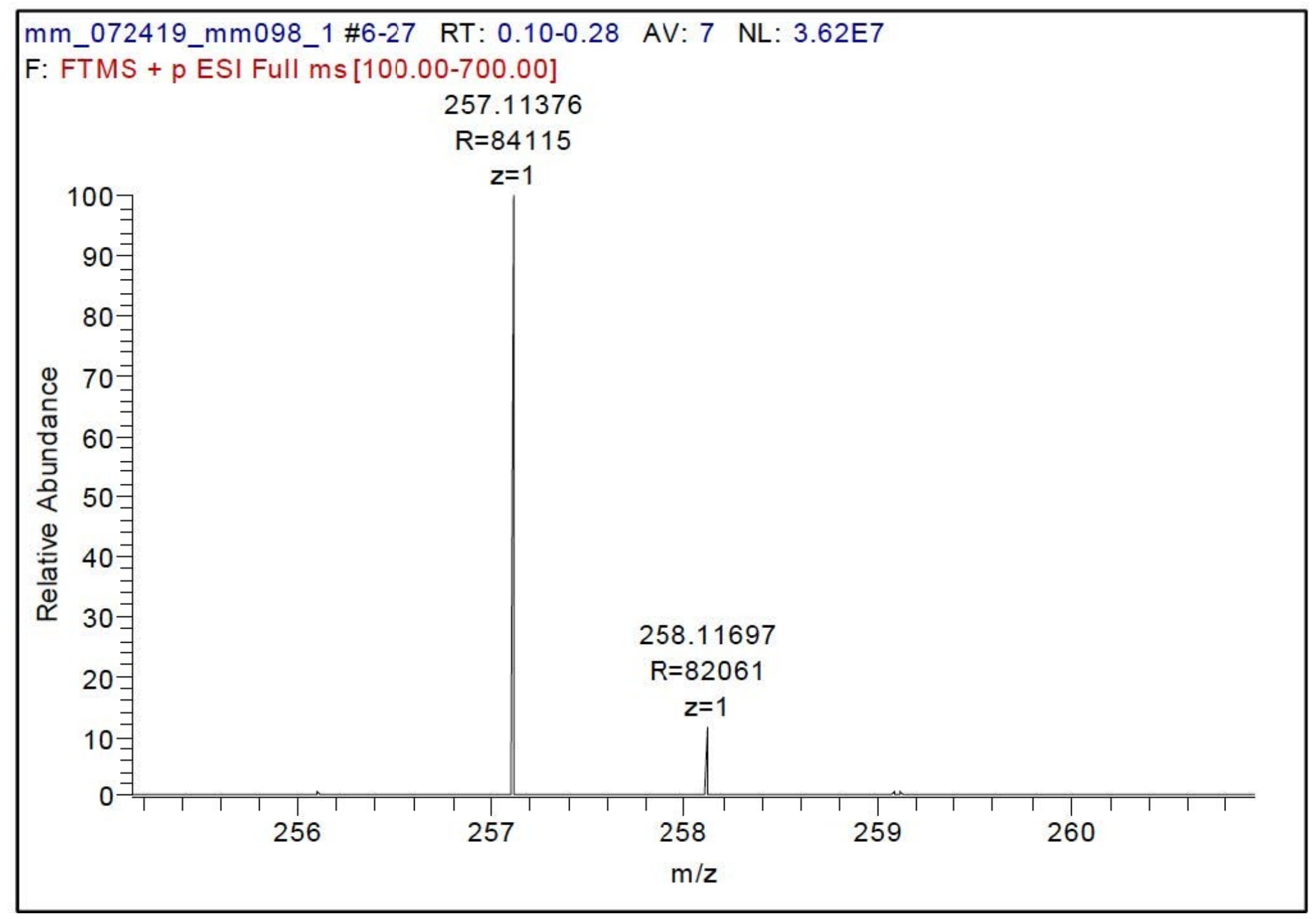

Fig. 23S HRMS (1:1 $\left.\mathrm{H}_{2} \mathrm{O} / \mathrm{ACN}\right)$ of of $\mathrm{NRH}(\mathbf{1})$. 


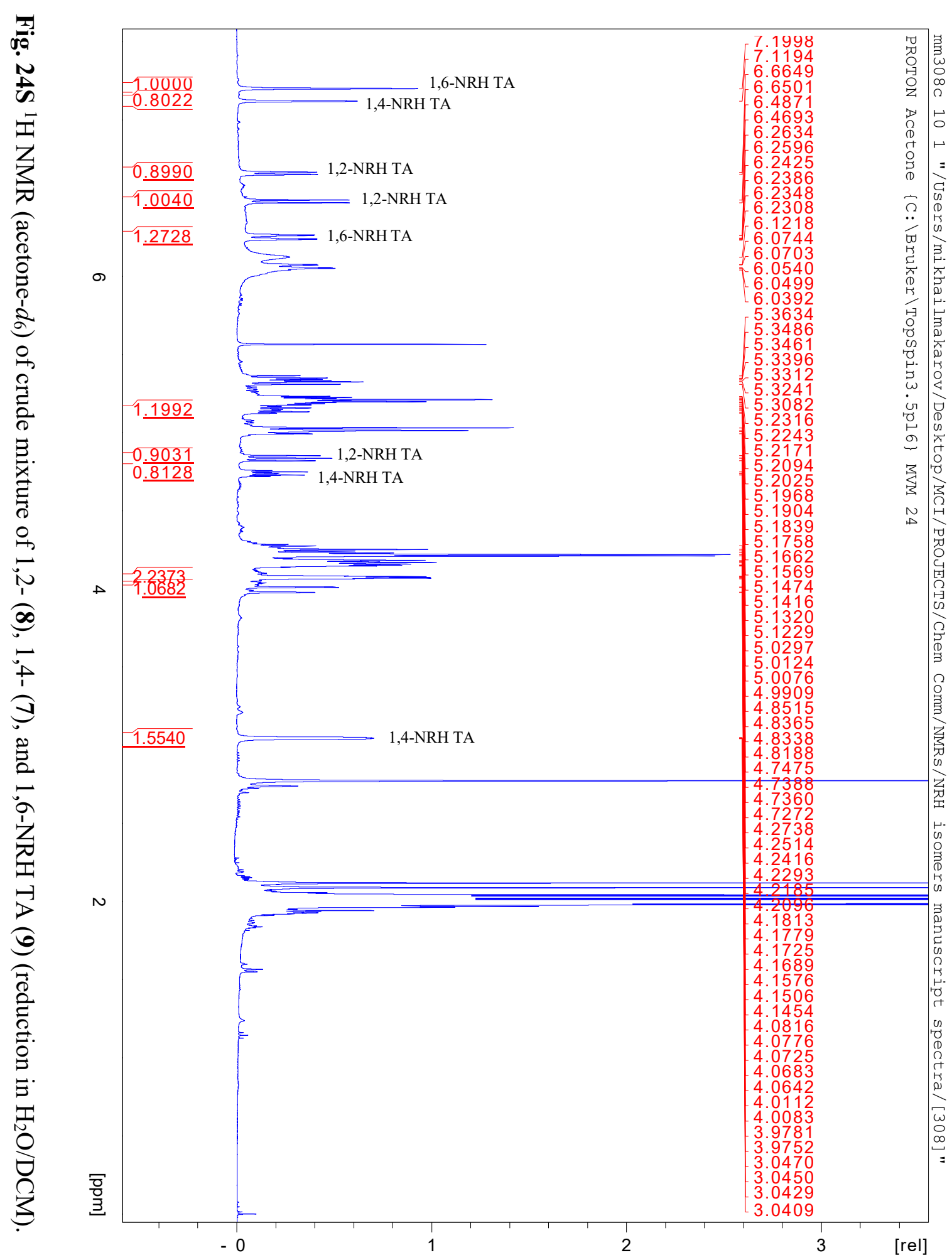


Sample: mm308

Column: Silica 40g

Flow Rate: $25 \mathrm{ml} / \mathbf{m i n}$

Equilibration Volume: $1922 \mathrm{ml}$

Initial Waste: $0.0 \mathrm{ml}$

Air Purge: 0.0 min

Solvent $A^{\star}$ : Hexanes, then EtoAc

Solvent $B^{\star}$ : EtOAc, then EtOH
Conbiflash NEXTGEN

Friday 26 April 2019 05:21PM

Peak Tube Volume: Max.

Non-Peak Tube Volume: Max.

Loading Type: Iiquid

Wavelength 1 (red): 254nm

Peak Width: 2 min

Threshold: 0.20 AU

Wavelength 2 (purple): $350 \mathrm{~nm}$

${ }^{\star}$ Run Notes: Chromatography was started with solvent A being hexanes and solvent B being EtOAc up to point X; after this point solvent A was EtOAc and solvent B was EtOH.
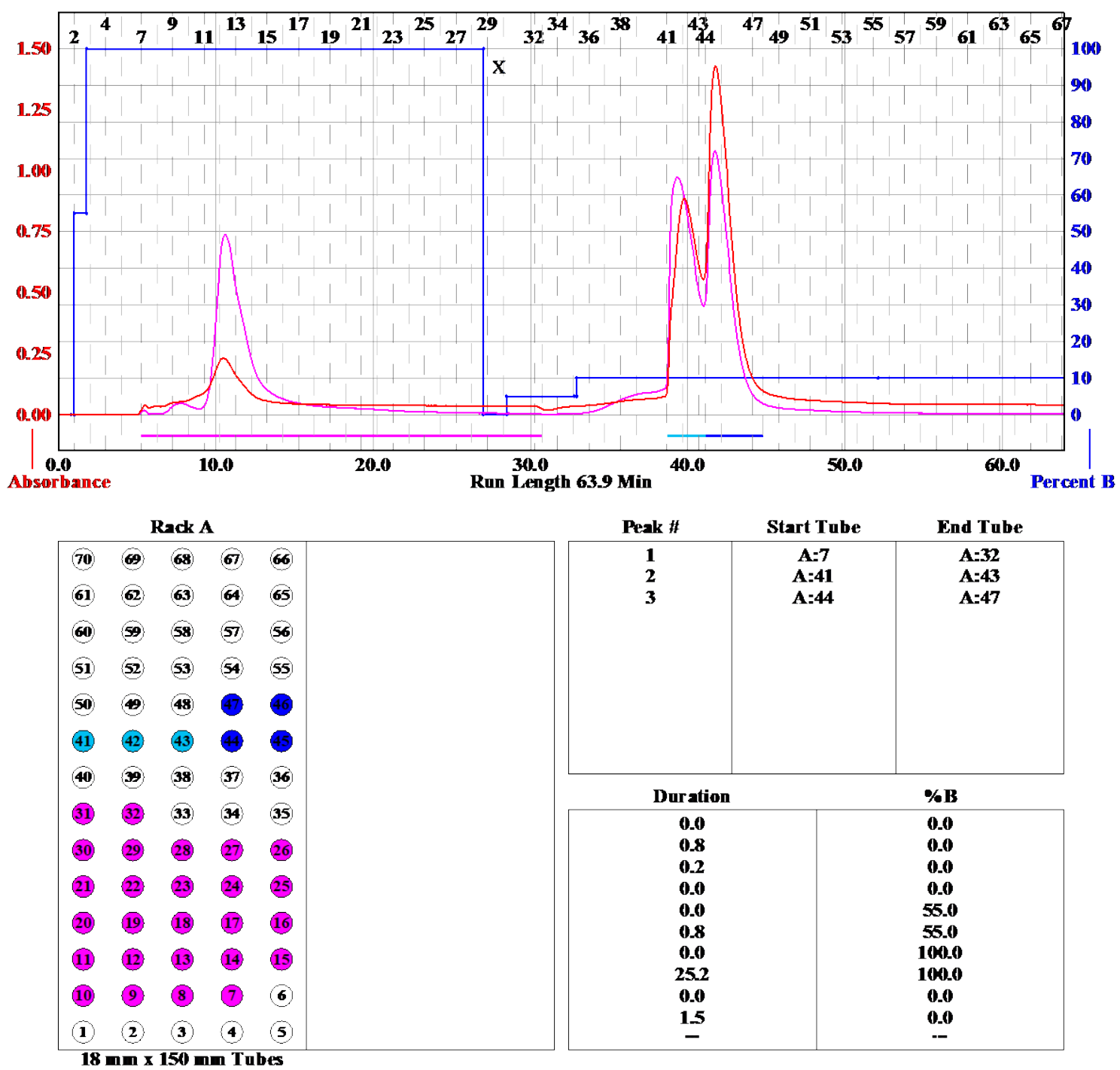

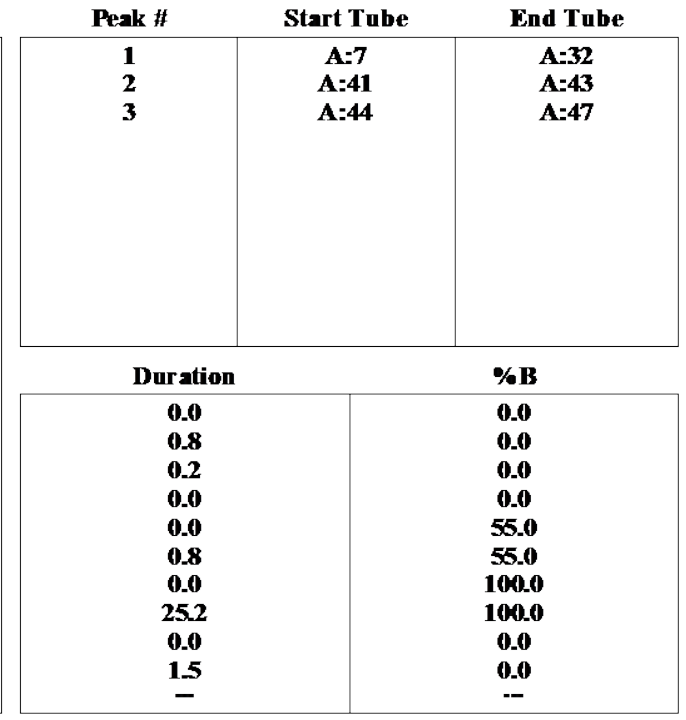

Page 1 of 1

Fig. 25S Data on MPLC (Teledyne) chromatographic purification of crude mixture of 1,2- (8), 1,4- (7), and 1,6-NRH TA (9) (reduction in $\mathrm{H}_{2} \mathrm{O} / \mathrm{DCM}$ ). 


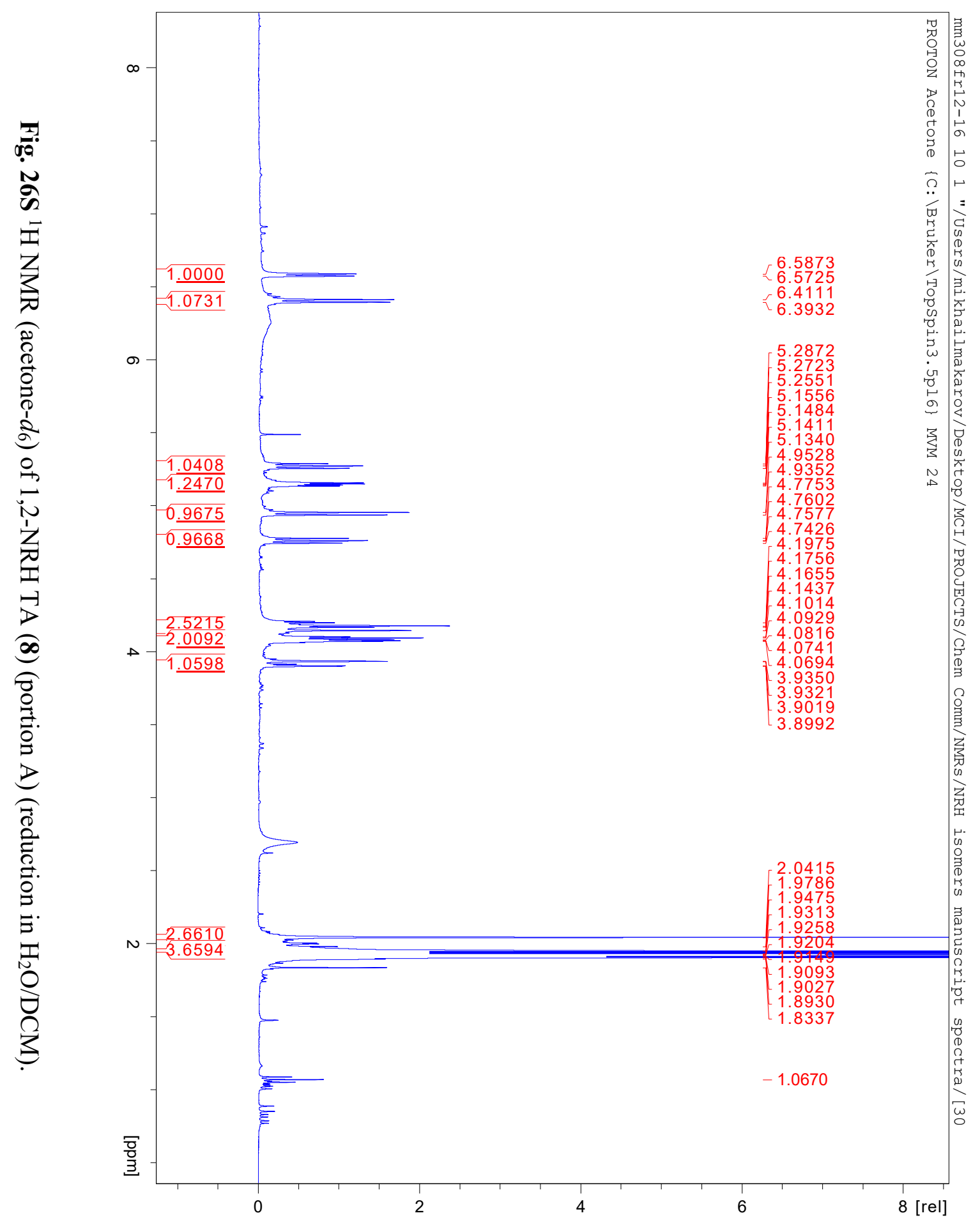




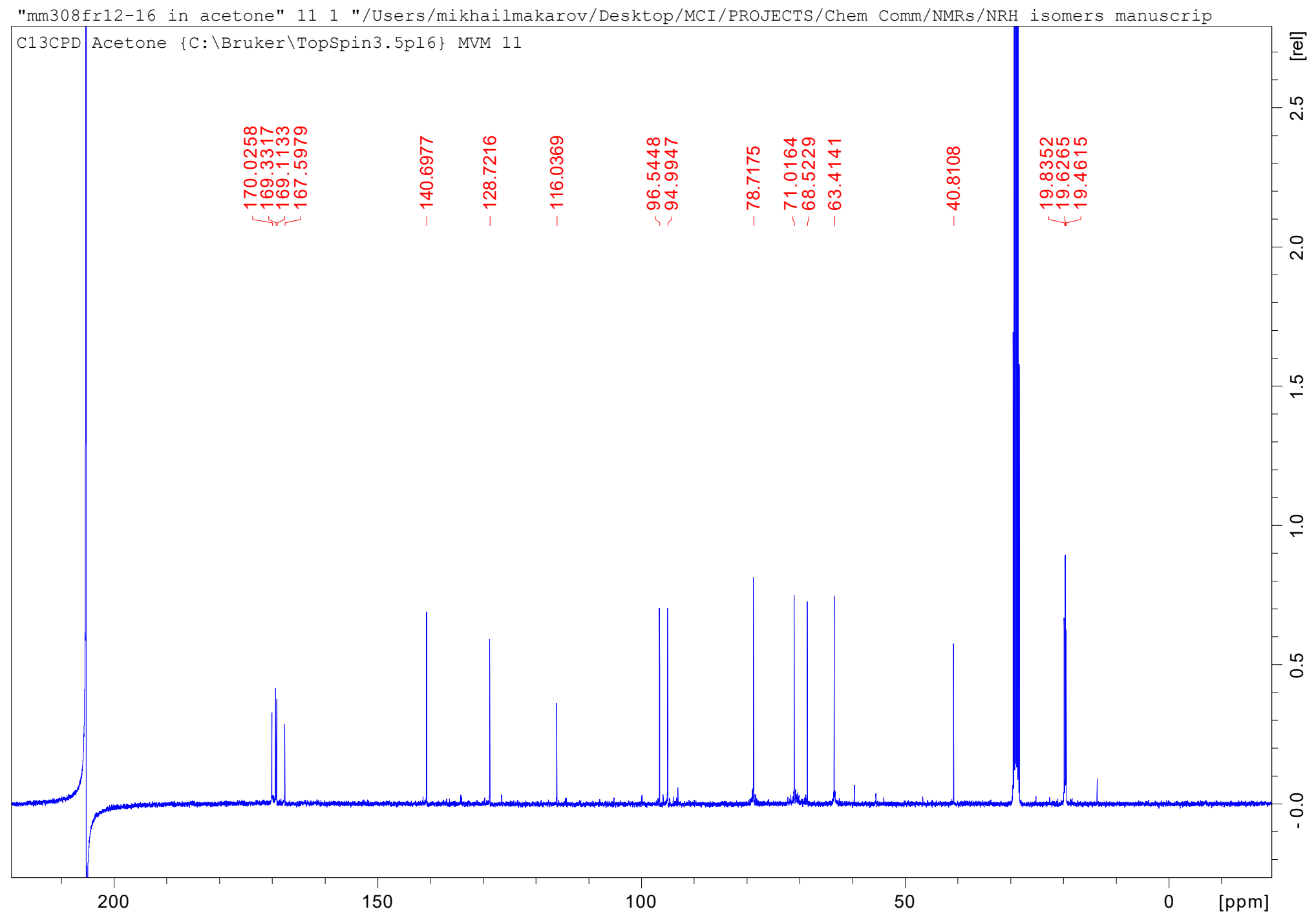

Fig. 27S ${ }^{13} \mathrm{C}$ NMR (acetone- $d_{6}$ ) of 1,2-NRH TA (8) (portion A) (reduction in $\mathrm{H}_{2} \mathrm{O} / \mathrm{DCM}$ ). 
D:LMSQ_Datalmm 042919 mm308a_600mass_02

04/29/19 16:00:10

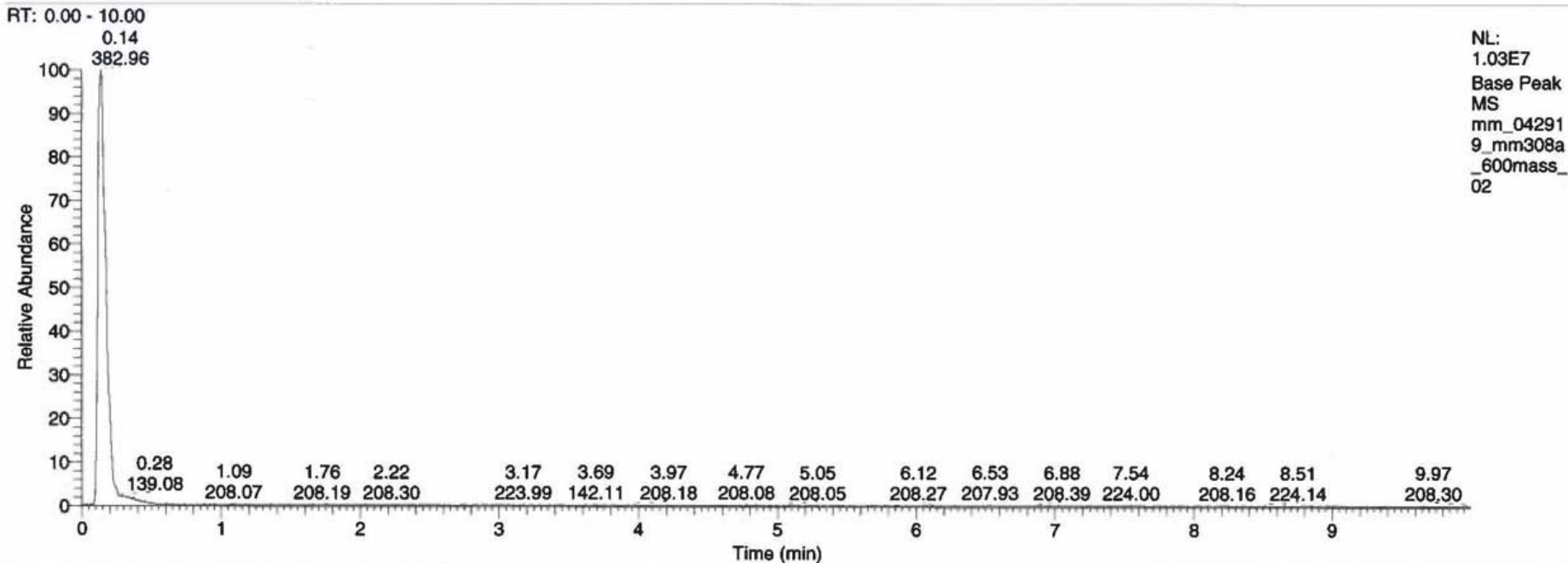

mm_042919_mm308a_600mass_02 \#11-26 RT: 0.09-0.22 AV: 16 NL: 4.20E6

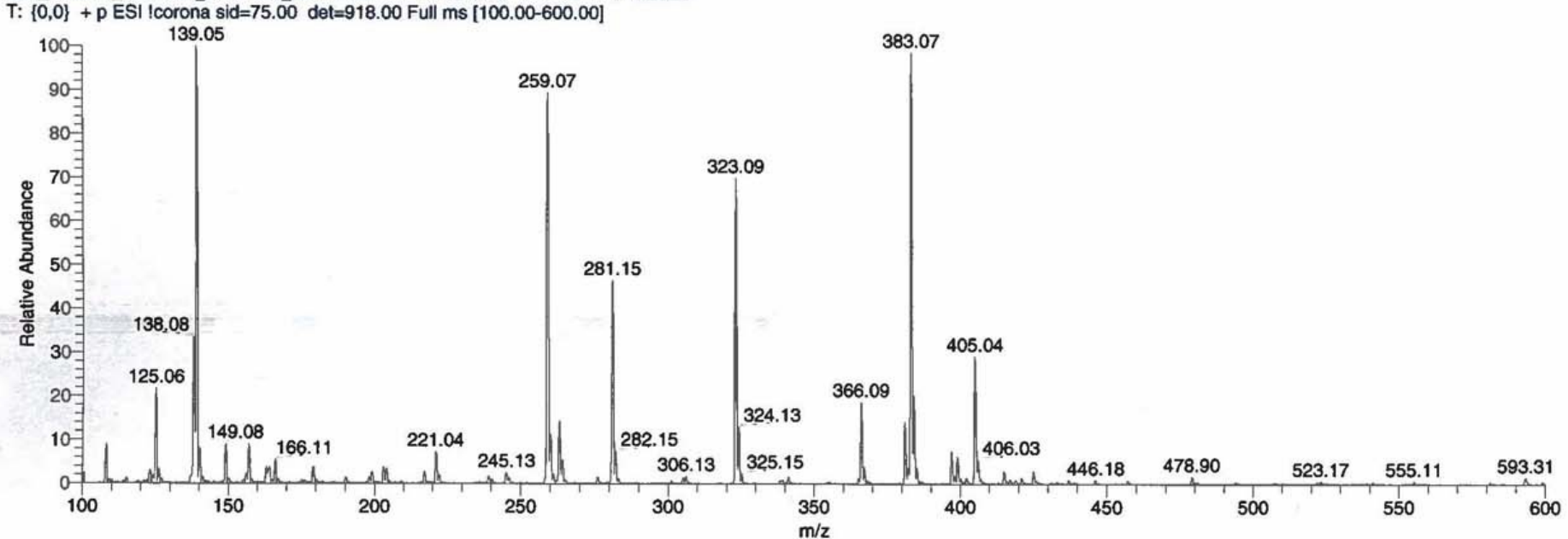

Fig. 28S MS (1:1 $\left.\mathrm{H}_{2} \mathrm{O} / \mathrm{ACN}\right)$ of 1,2-NRH TA (8) 
mm_050219_mm308a_1 \#7-27 RT: 0.09-0.27

F: FTMS + p ESI Full ms [100.00-500.00]

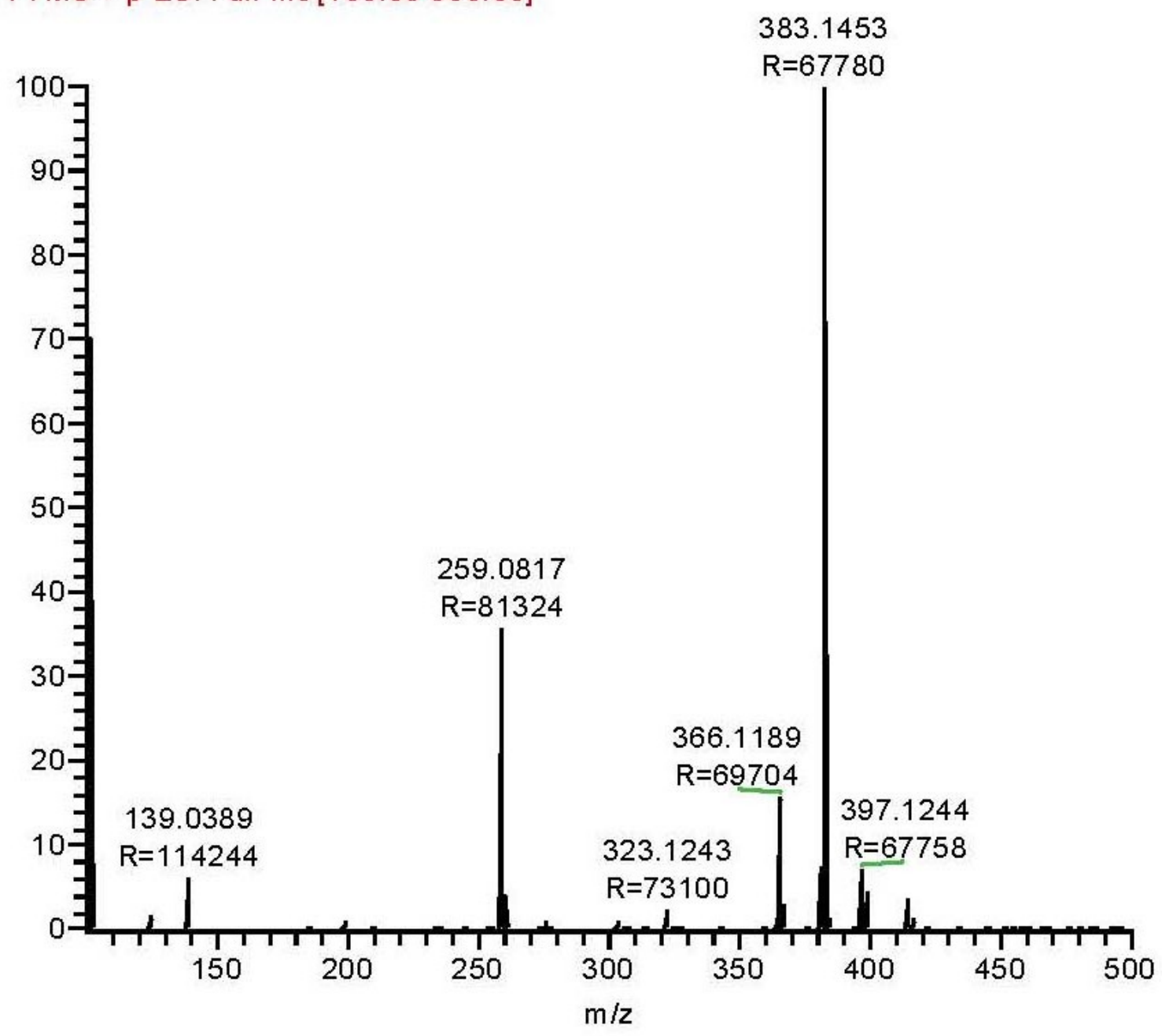

Fig. 29S HRMS (1:1 $\left.\mathrm{H}_{2} \mathrm{O} / \mathrm{ACN}\right)$ of 1,2-NRH TA (8): full scan. 


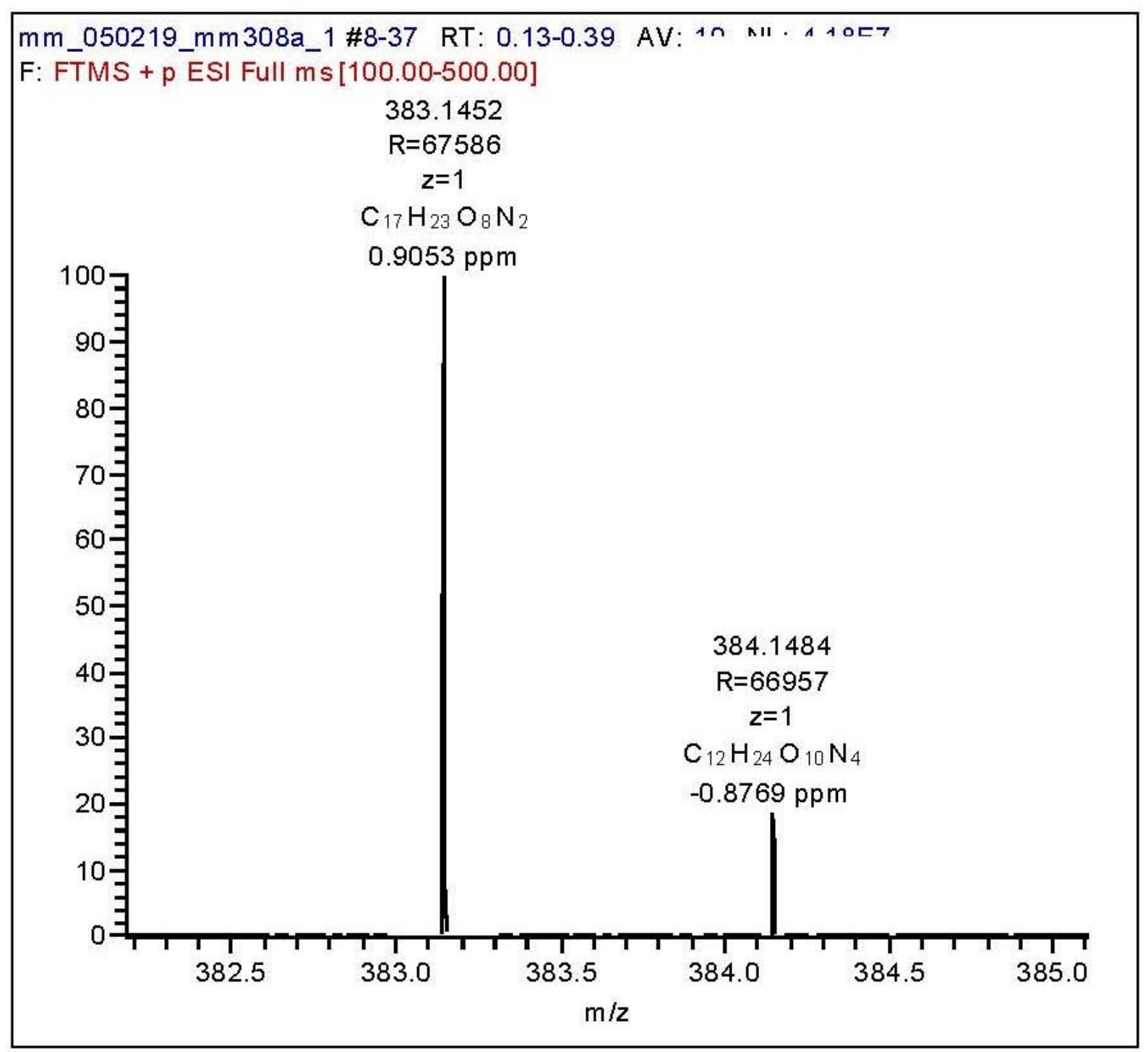

Fig. 30S HRMS (1:1 $\left.\mathrm{H}_{2} \mathrm{O} / \mathrm{ACN}\right)$ of 1,2-NRH TA (8): parent peak. 


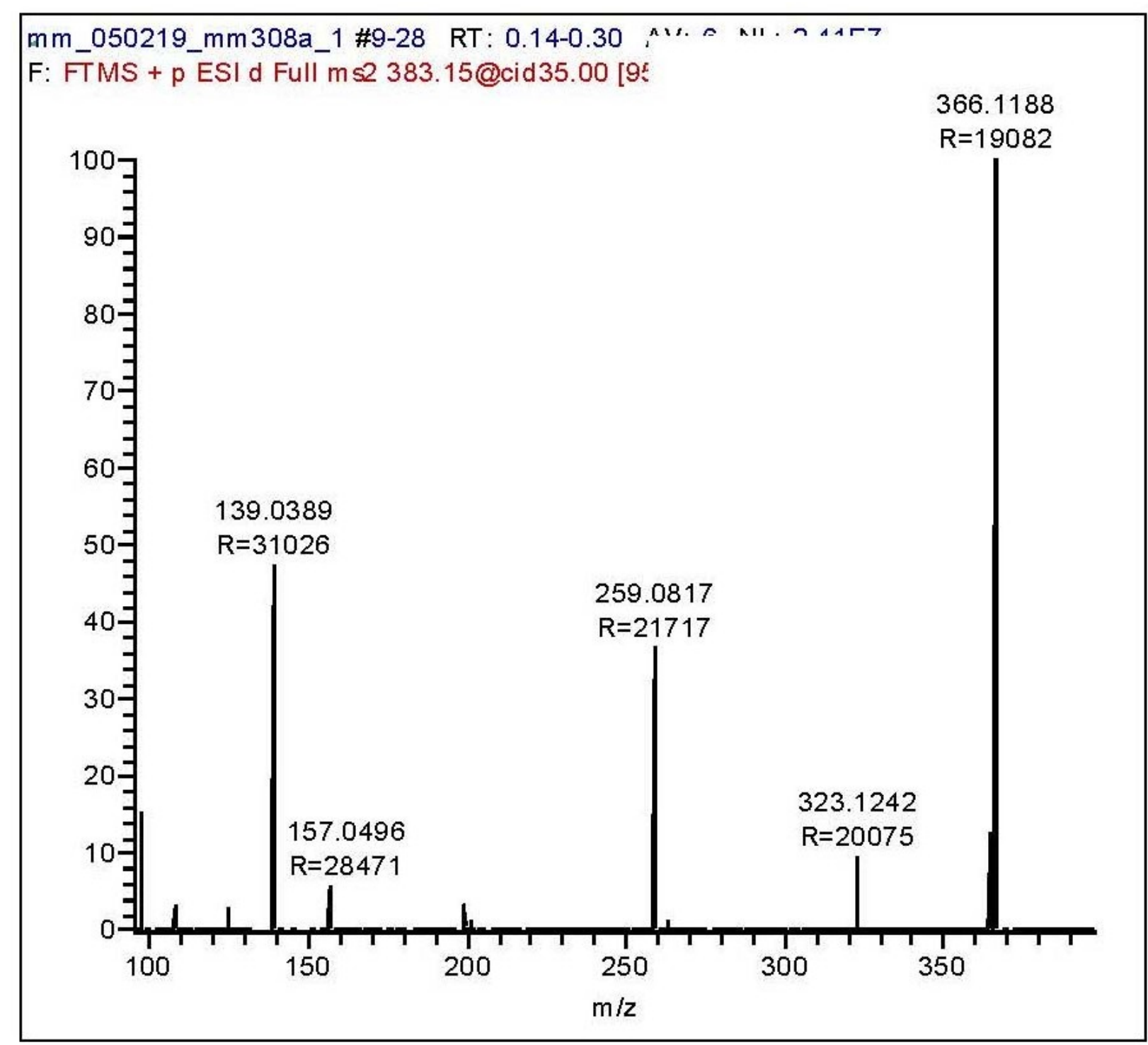

Fig. 31S HRMS (1:1 $\left.\mathrm{H}_{2} \mathrm{O} / \mathrm{ACN}\right)$ of 1,2-NRH TA (8): fragmentation of parent peak. 


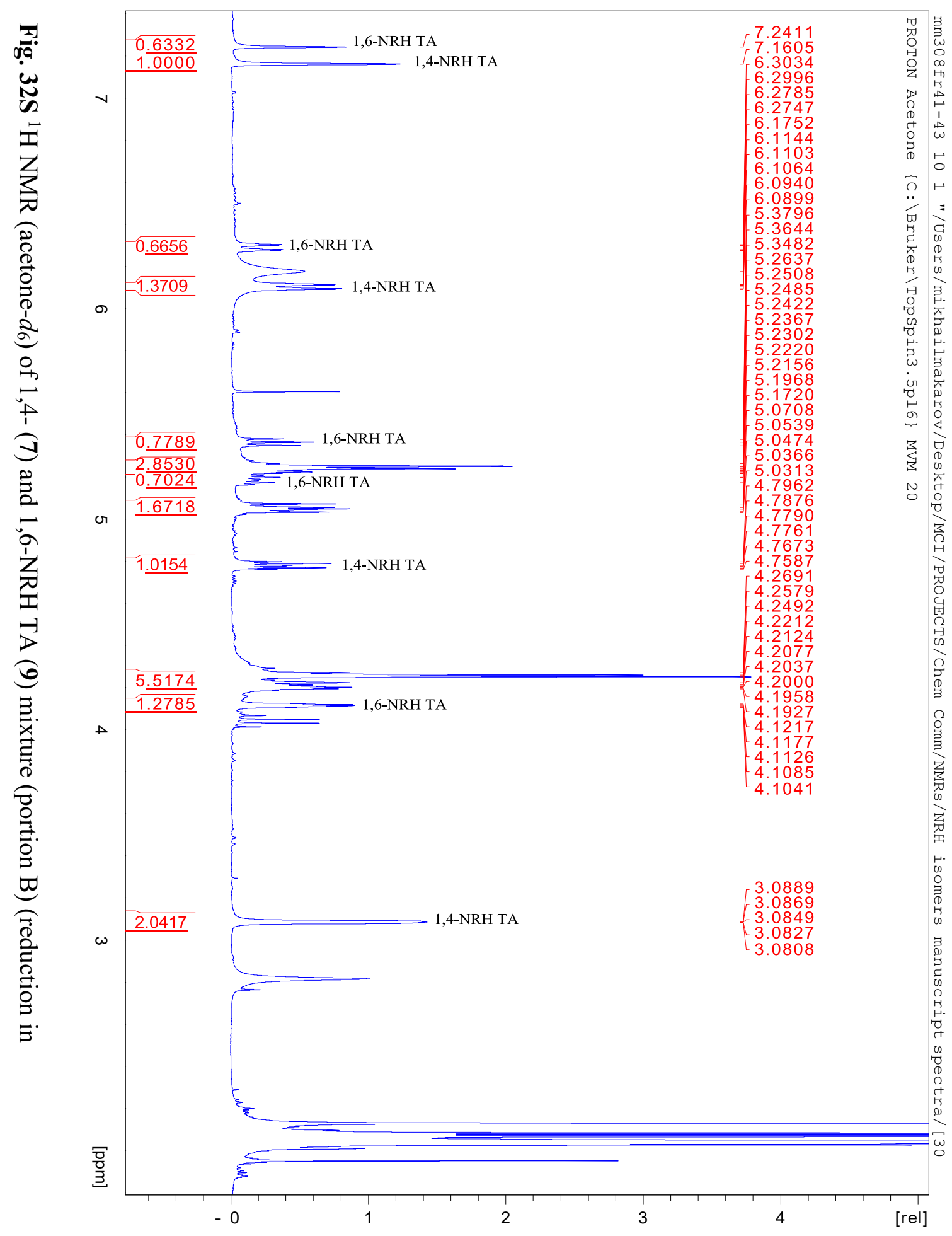




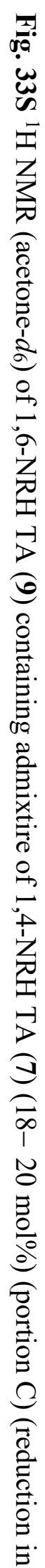

7.2410

$-7.1599$

- 6.3039

6.3002
6.2791

6.2791
6.2753

5.3796

5. 3641

5. 3482

5.2380
5.2300

5.2239

5. 1966

5. 1718

5.0706
5.0538

4.7958

4.7873

4.7785

4.7757

4.7668

4.7583

4.2691
4.2585

4.2496

4.2211
4.2123

4.1213

4.1124

4.1083

4.1040

3.0871

3.0851
3.0829
3.0809

3.0809
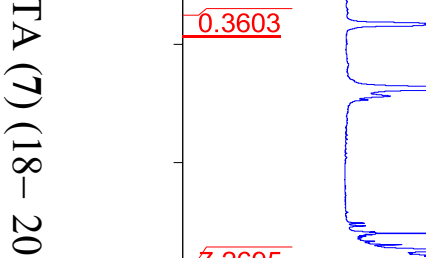

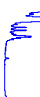

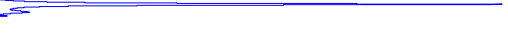

?

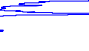




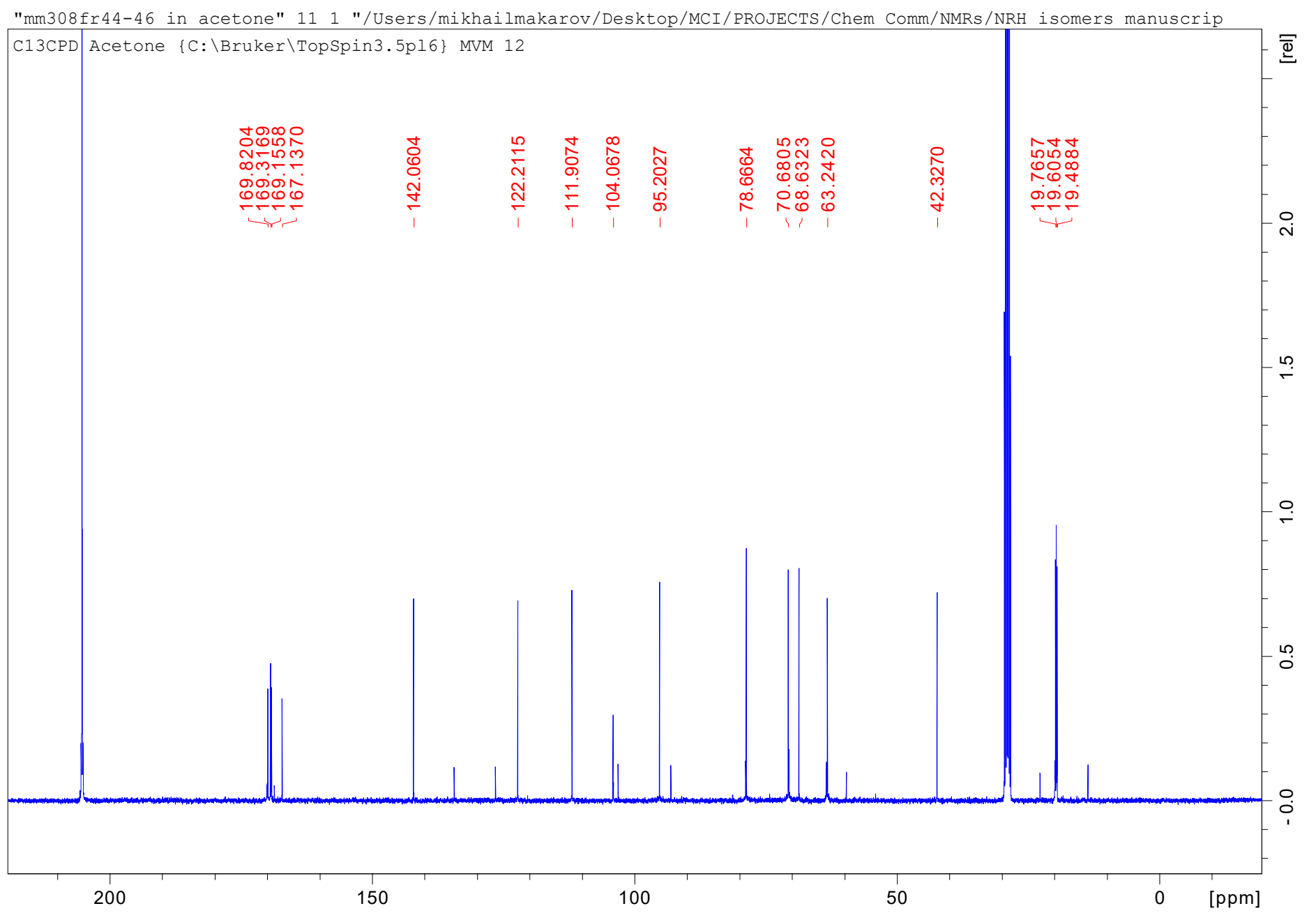

Fig. 34S ${ }^{13} \mathrm{C}$ NMR (acetone- $d_{6}$ ) of 1,6-NRH TA (9) containing admixtire of 1,4-NRH TA (7) (18-20 mol\%) (portion C) (reduction in $\mathrm{H}_{2} \mathrm{O} / \mathrm{DCM}$ ). 
D:IMSQ Datalmm_042919_mm308b_600mass_02

04/29/19 16:11:38

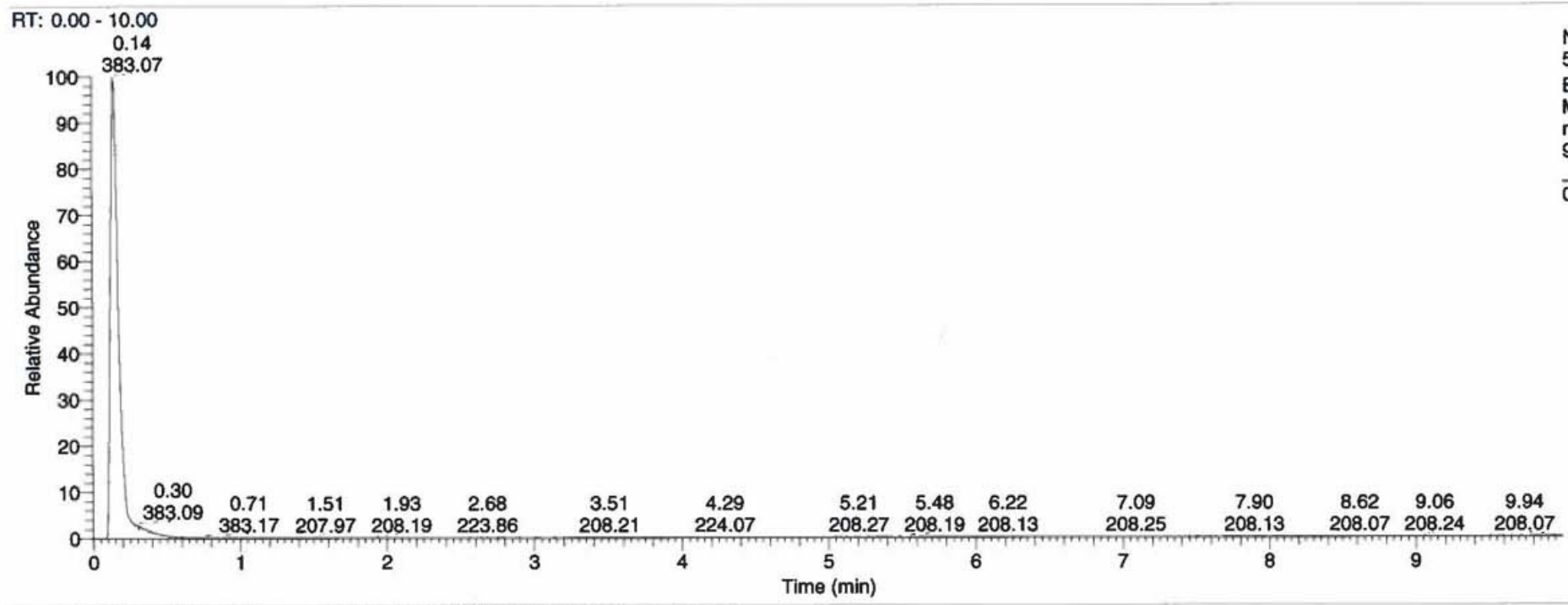

mm_042919 mm308b_600mass_02\#12-26 RT: 0.10-0.22 AV: 15 NL: 2.29E7 T: $\{0,0\}+p$ ESI lcorona sid $=75.00$ det $=918.00$ Full $\mathrm{ms}[100.00-600.00]$

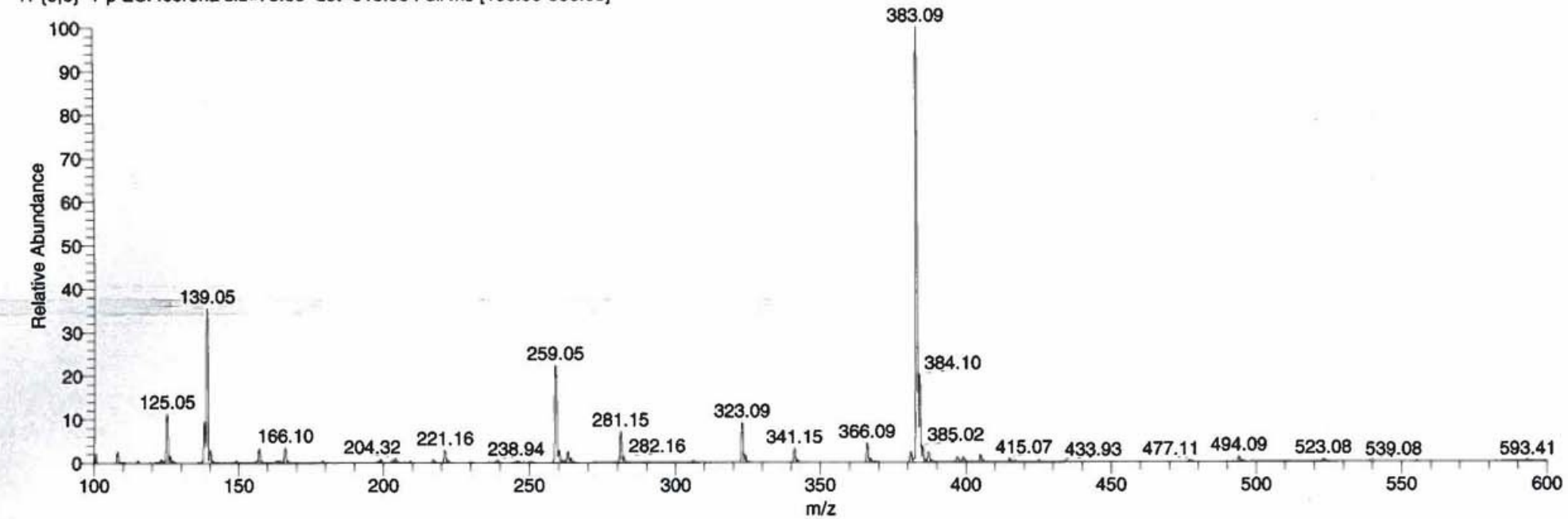

Fig. 35S MS (1:1 $\left.\mathrm{H}_{2} \mathrm{O} / \mathrm{ACN}\right)$ 1,6-NRH TA (9) 


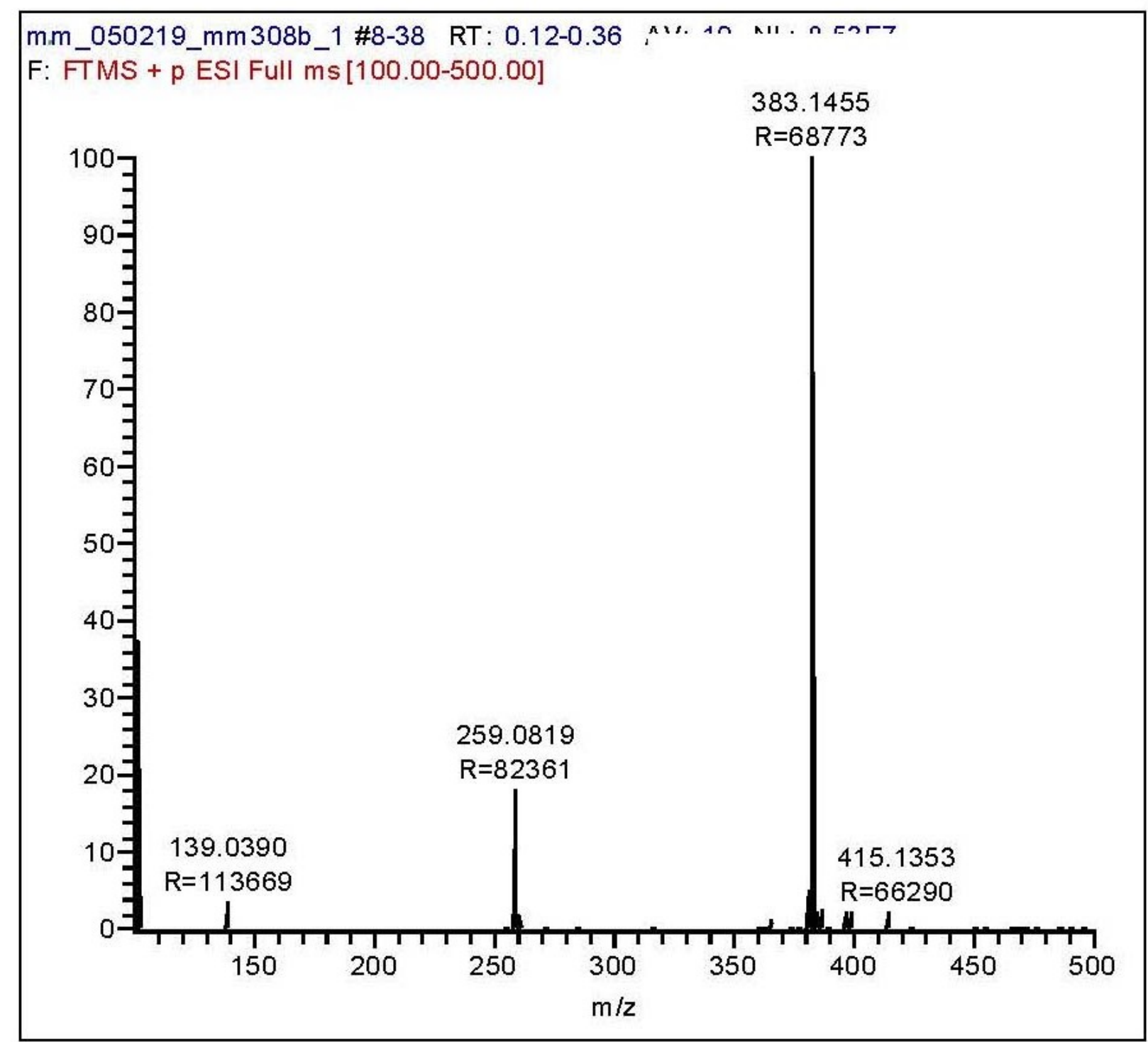

Fig. 36S HRMS (1:1 H2O/ACN) of 1,6-NRH TA (9): full scan. 


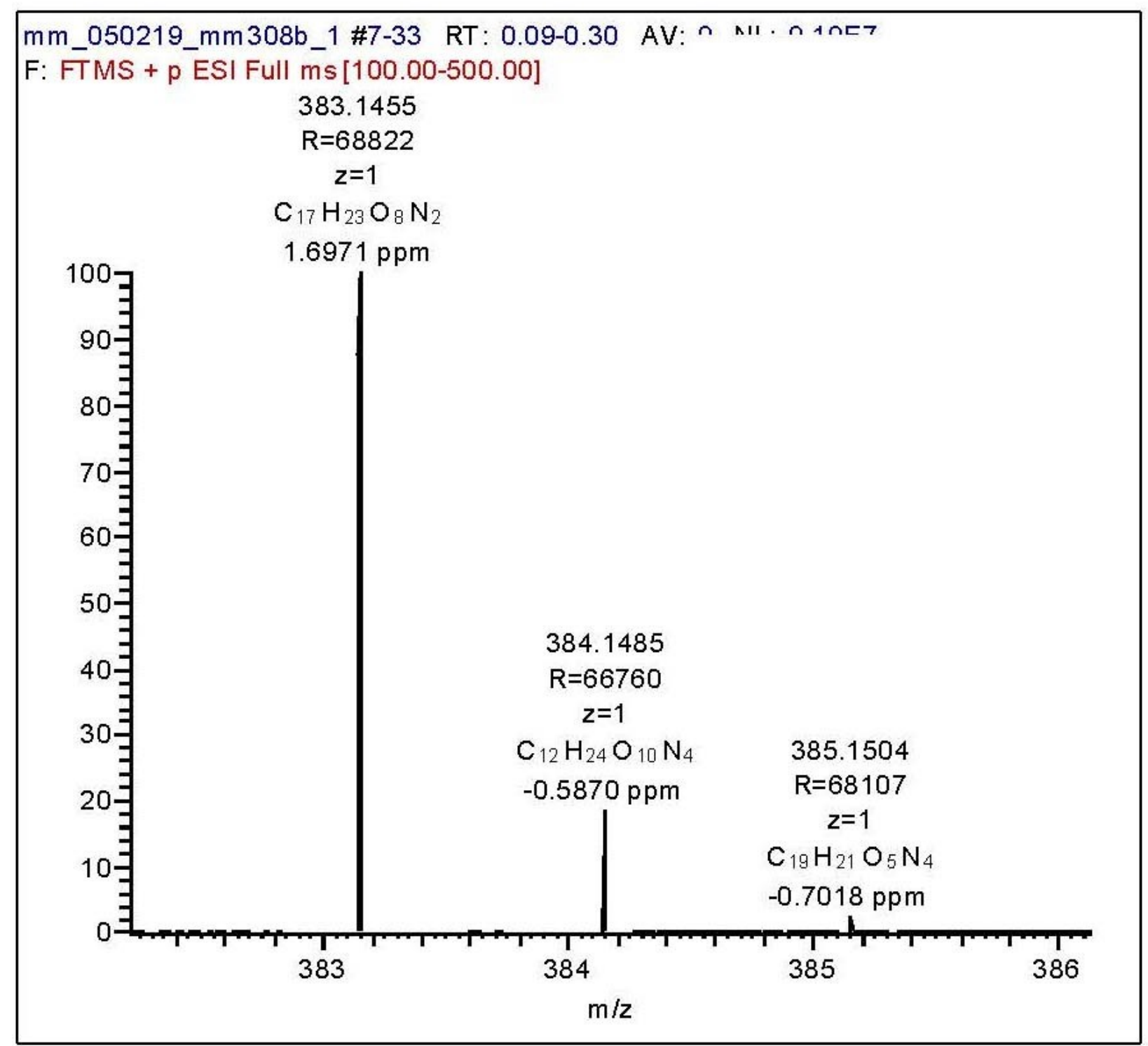

Fig. 37S HRMS (1:1 $\left.\mathrm{H}_{2} \mathrm{O} / \mathrm{ACN}\right)$ of 1,6-NRH TA (6): parent peak. 


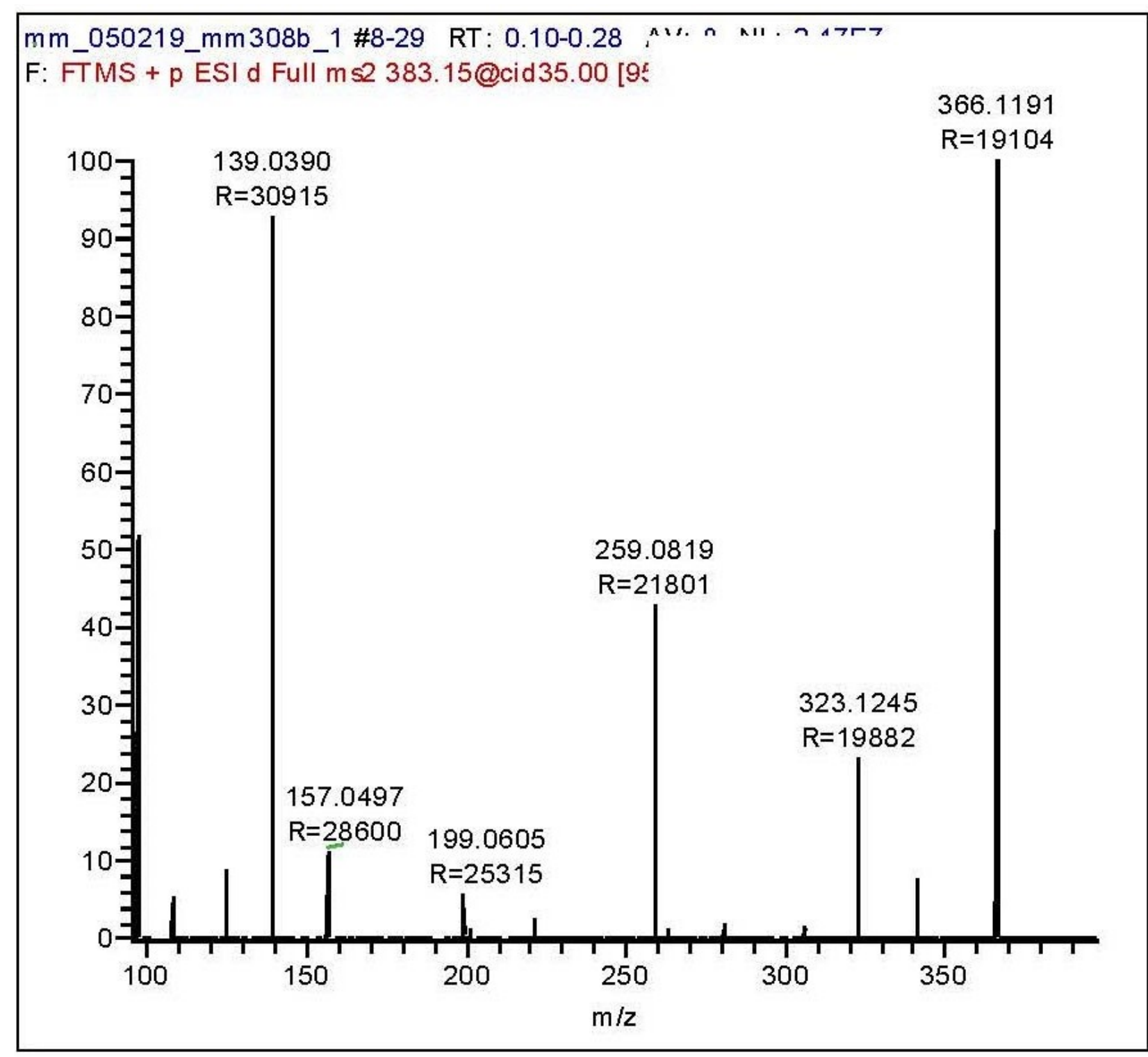

Fig. 38S HRMS $\left(1: 1 \mathrm{H}_{2} \mathrm{O} / \mathrm{ACN}\right)$ of 1,6-NRH TA (9): fragmentation of parent peak. 


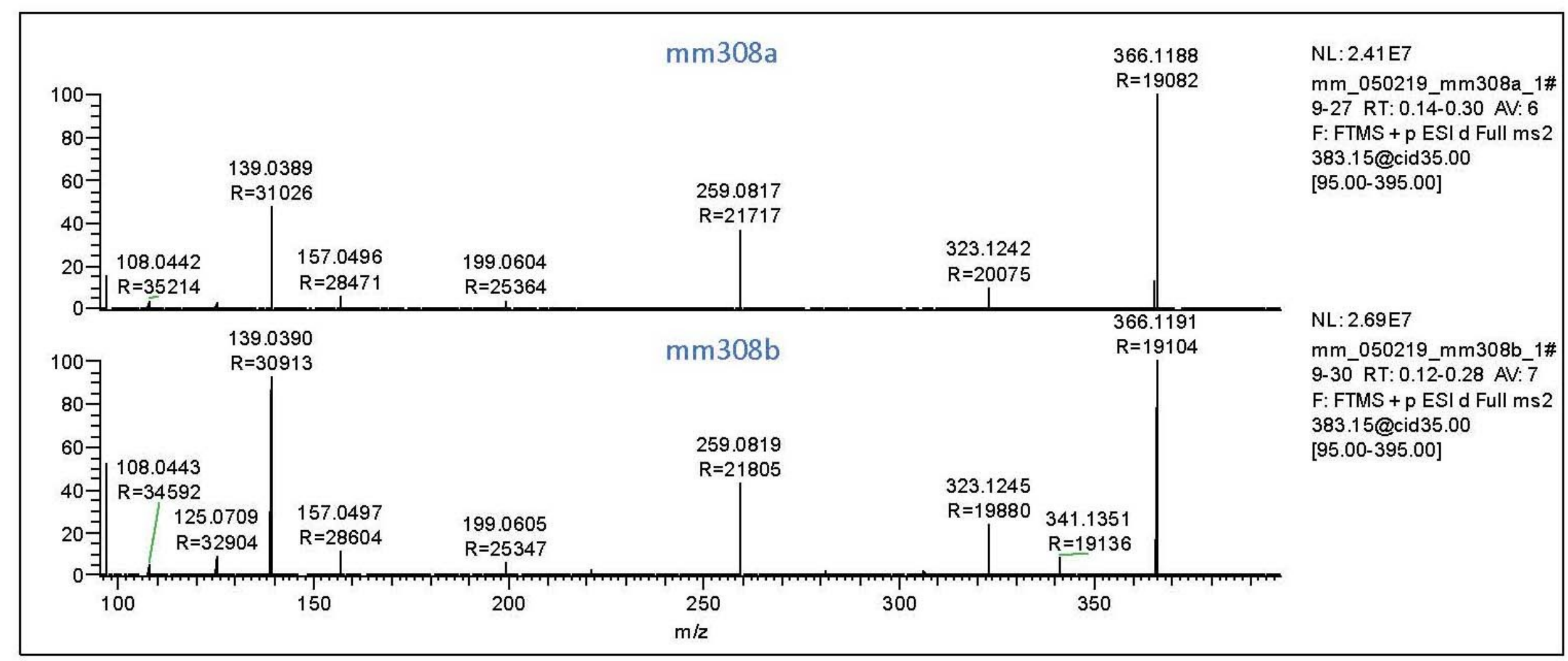

Fig. 39S Comparison of frafmentation of 1,2-NRH TA (8) (sample mm308a) and 1,6-NRH TA (9) (sample mm308b). 


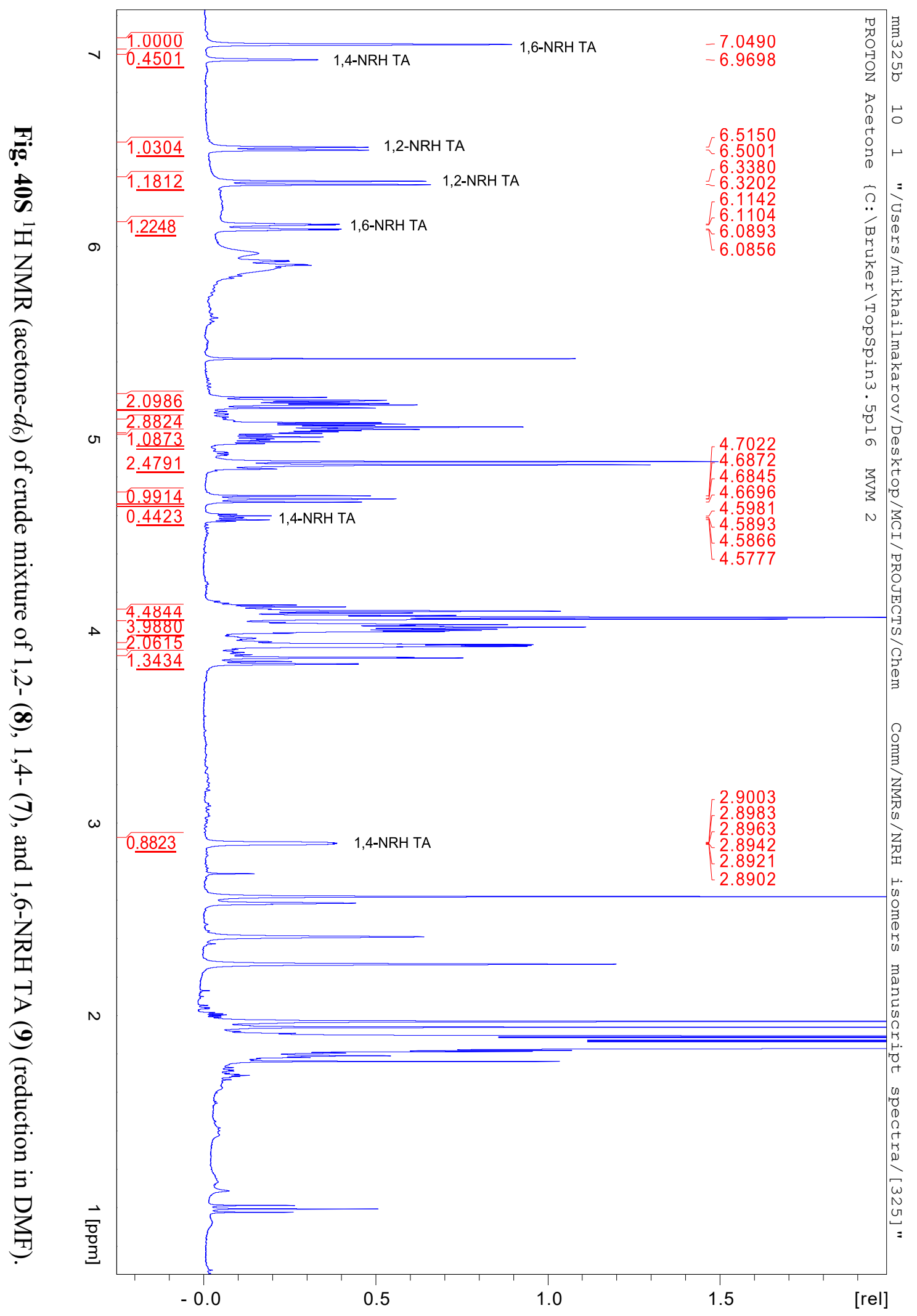




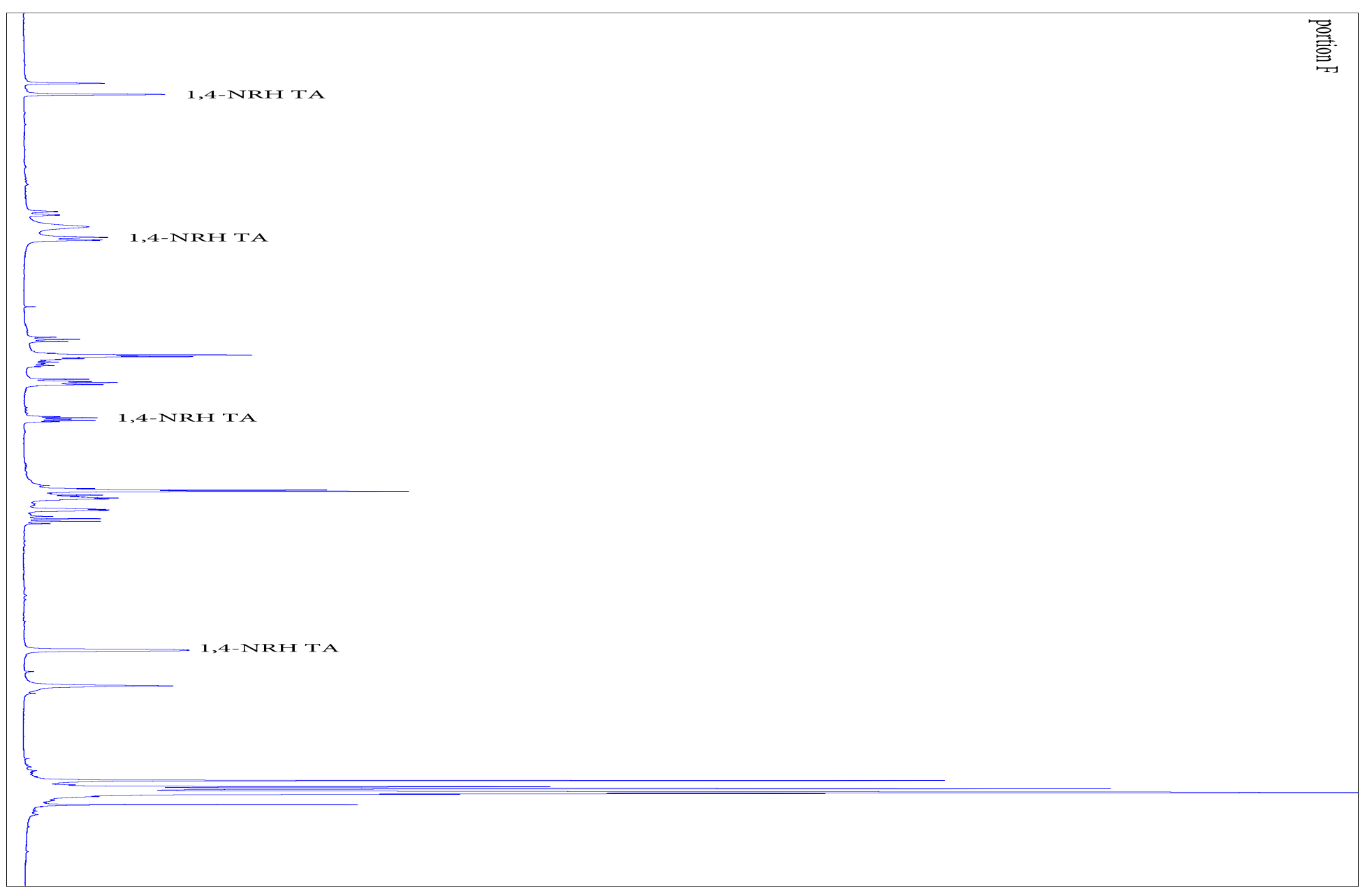

Fig. 41S Side-by-side comparison of ${ }^{1} \mathrm{H}$ NMRs (acetone- $d_{6}$ ) of portions C-F containg 1,6-NRH TA (9) and variable amounuts of 1,4-NRH TA (7) (reduction in DMF) 


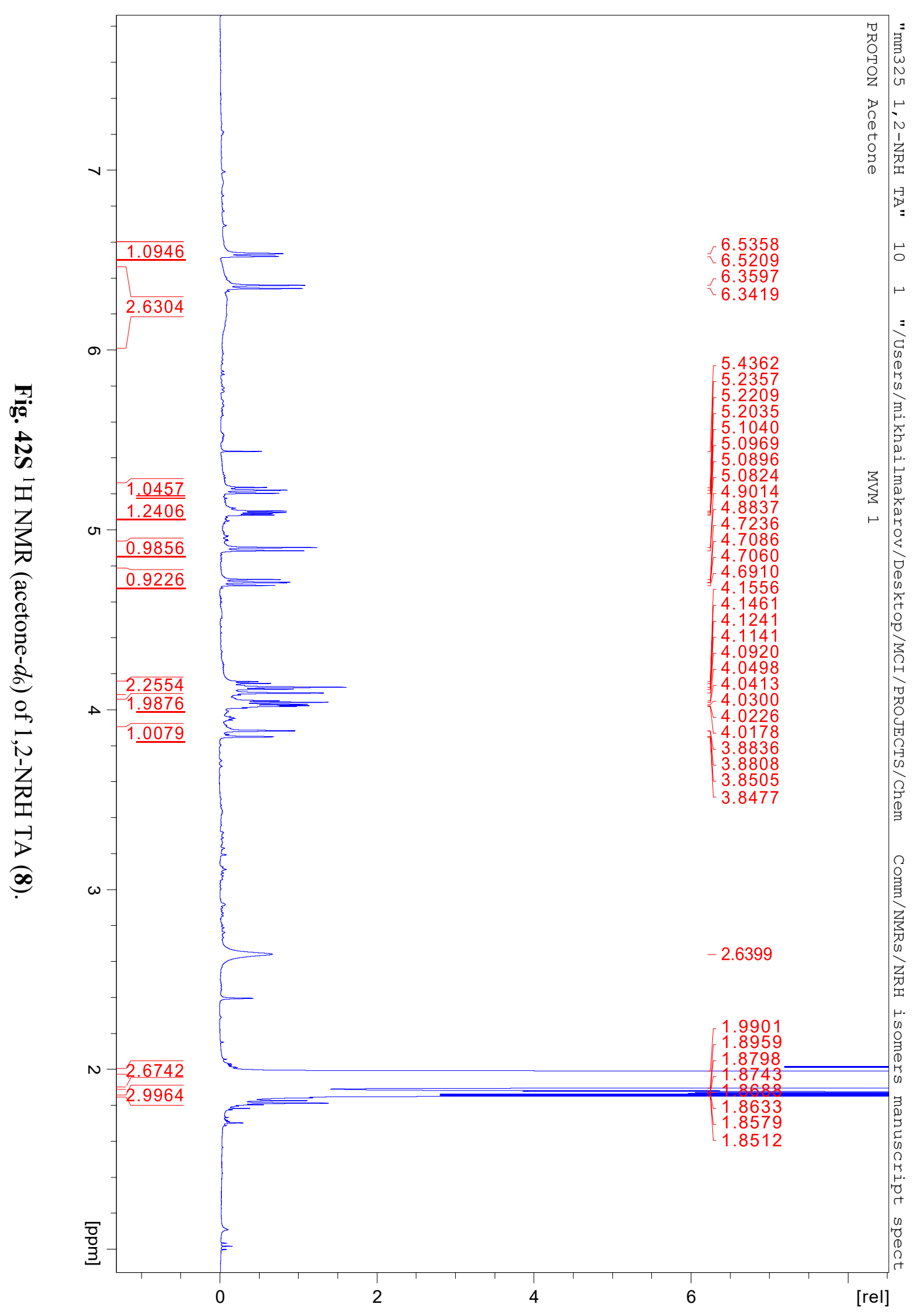




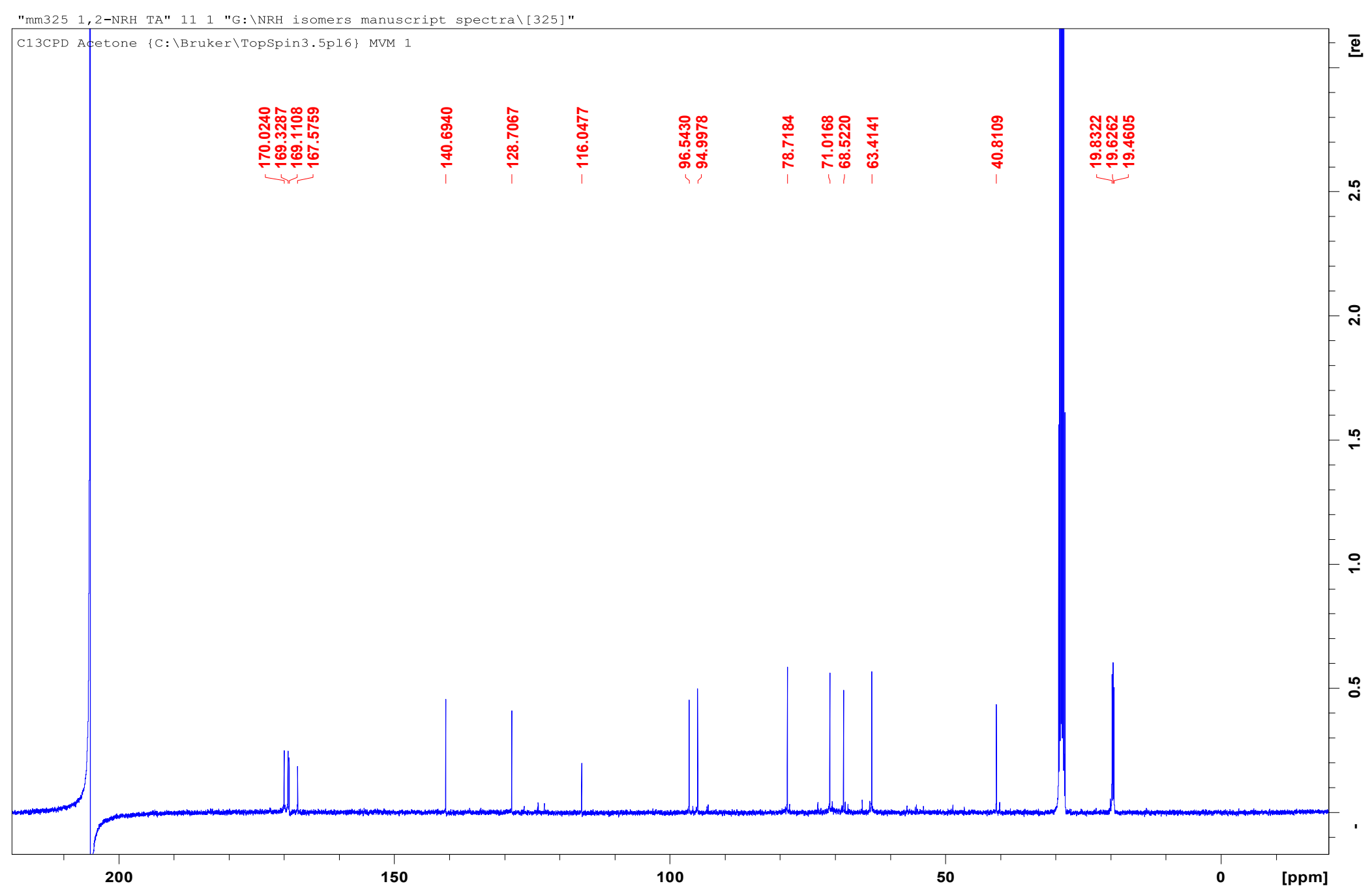

Fig. 43S ${ }^{13} \mathrm{C}$ NMR (acetone- $d_{6}$ ) of 1,2-NRH TA (8). 


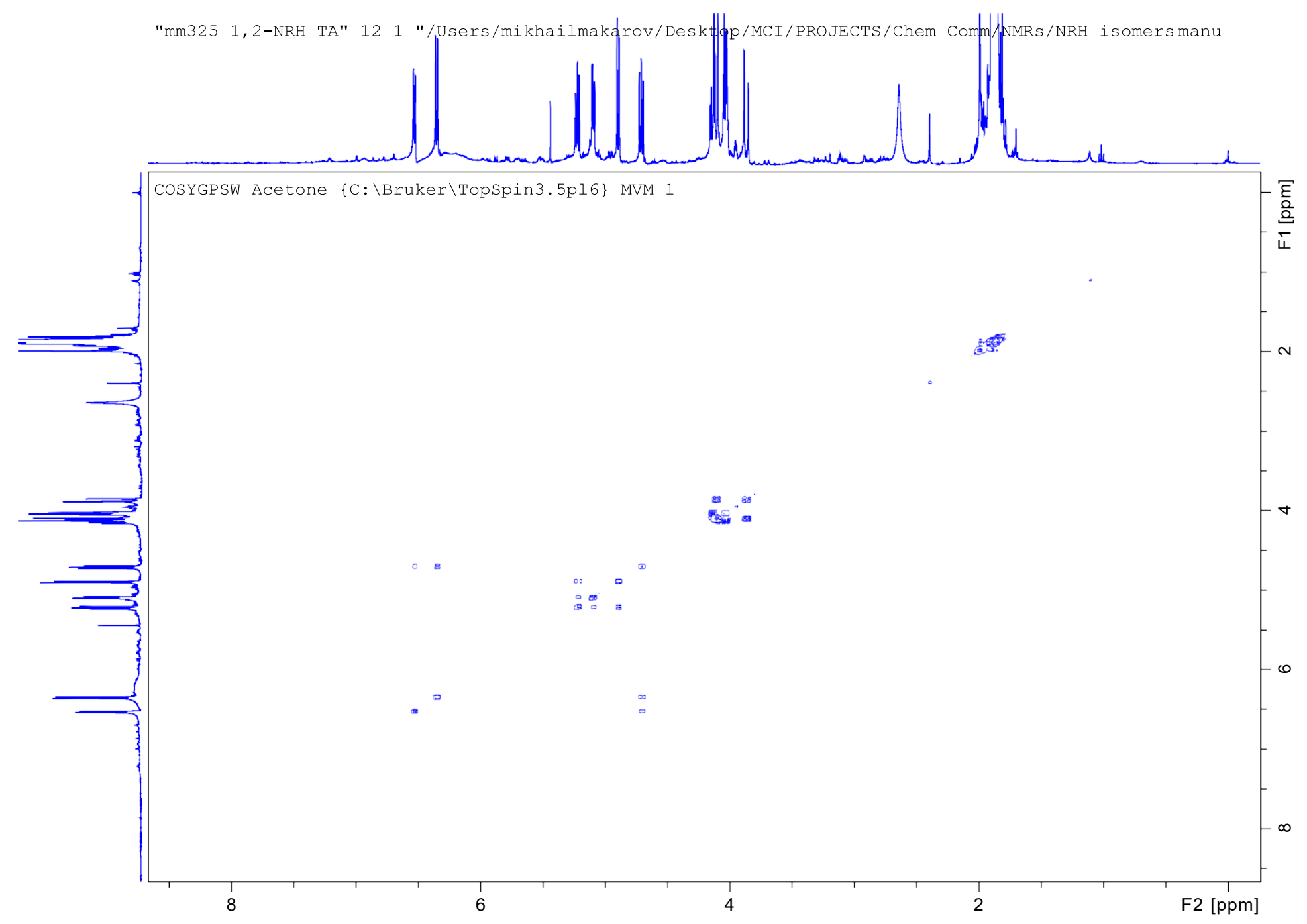

Fig. 44S ${ }^{1} \mathrm{H}-{ }^{1} \mathrm{H}$ correlation (COSY) NMR (acetone- $\left.d_{6}\right)$ of 1,2-NRH TA (8). 


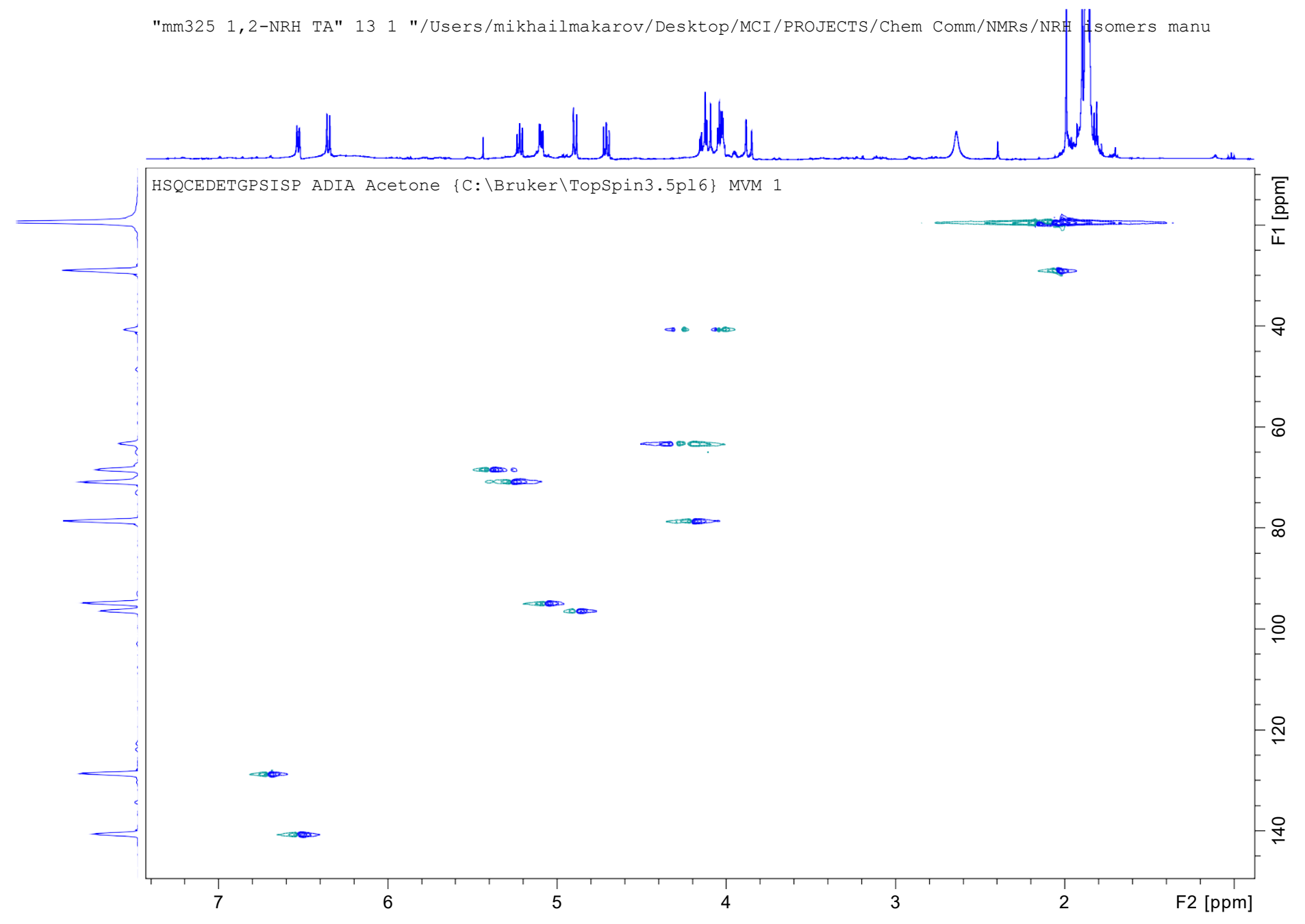

Fig. 45S ${ }^{1} \mathrm{H}-{ }^{13} \mathrm{C}$ correlation (HSQC) NMR (acetone- $d_{6}$ ) of 1,2-NRH TA (8). 


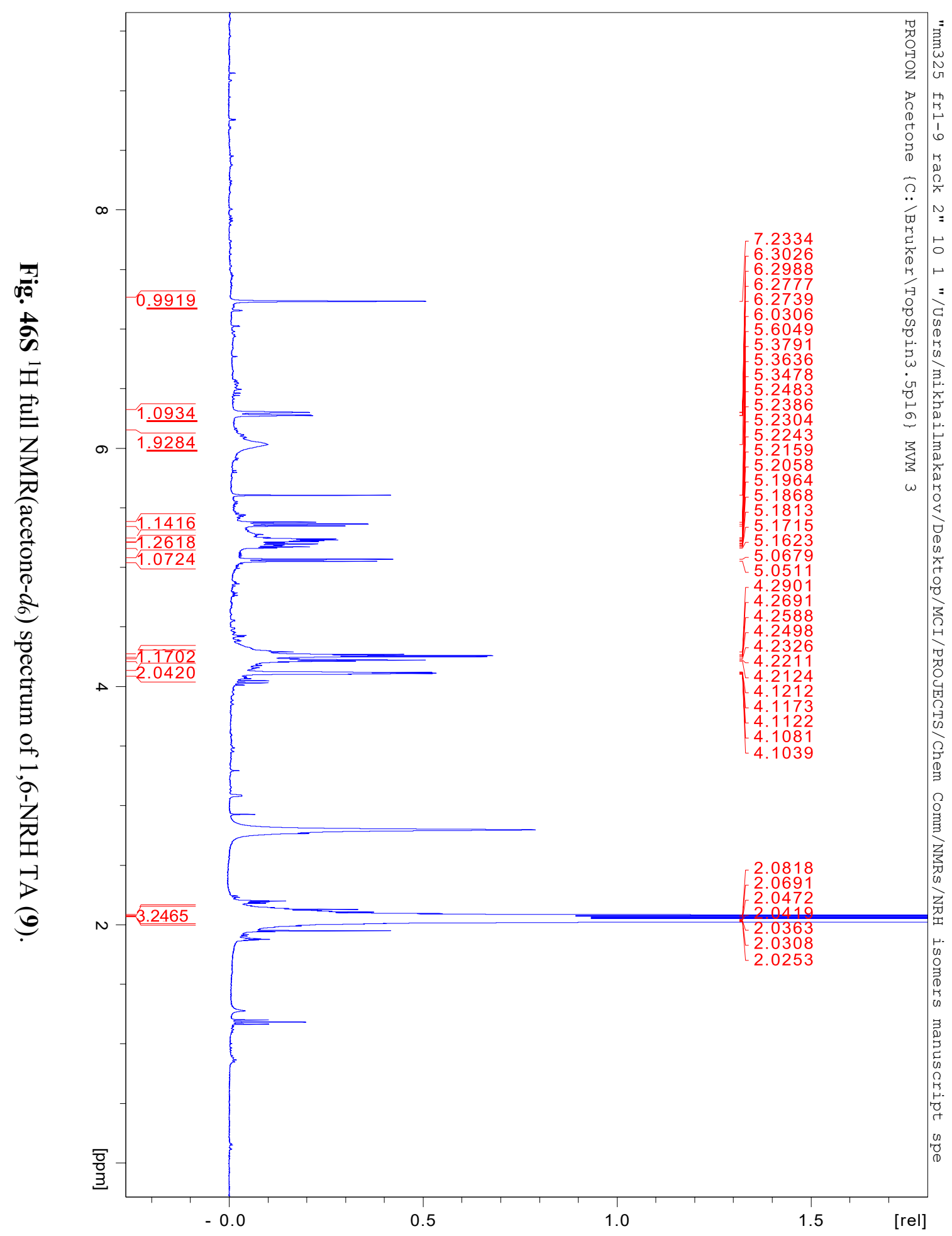




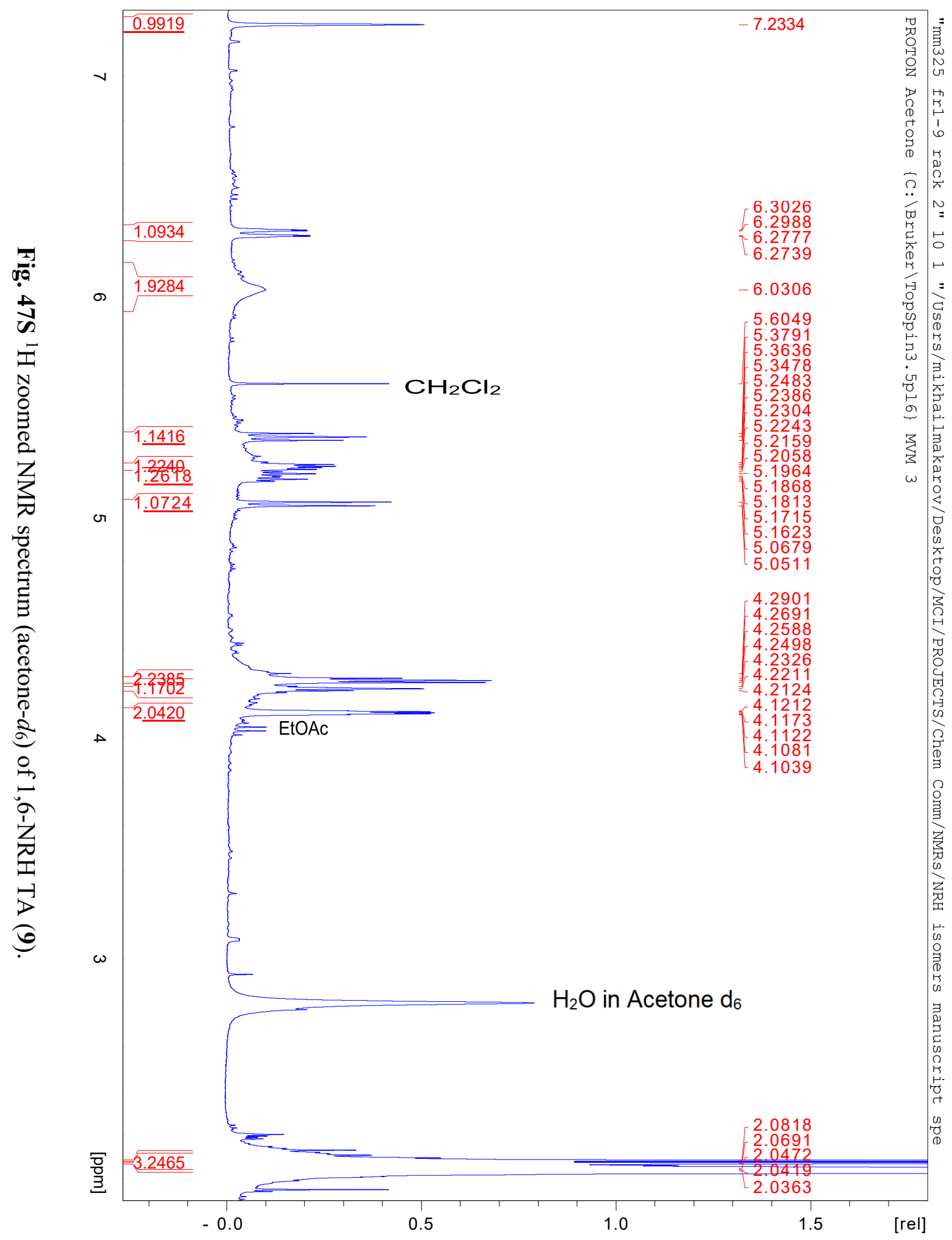




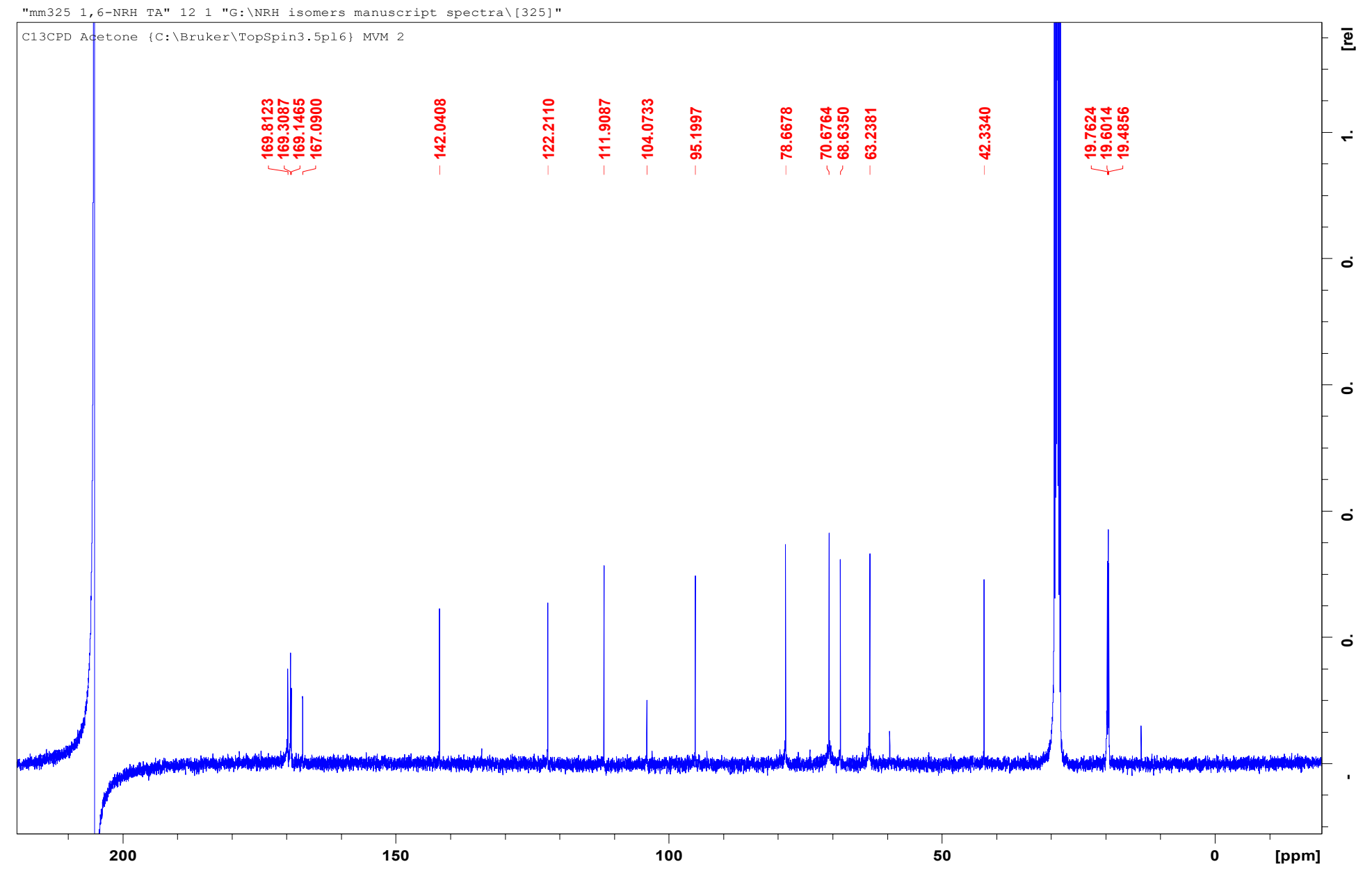

Fig. $48 \mathrm{~S}{ }^{13} \mathrm{C}$ NMR (acetone- $d_{6}$ ) of 1,6-NRH TA (9). 


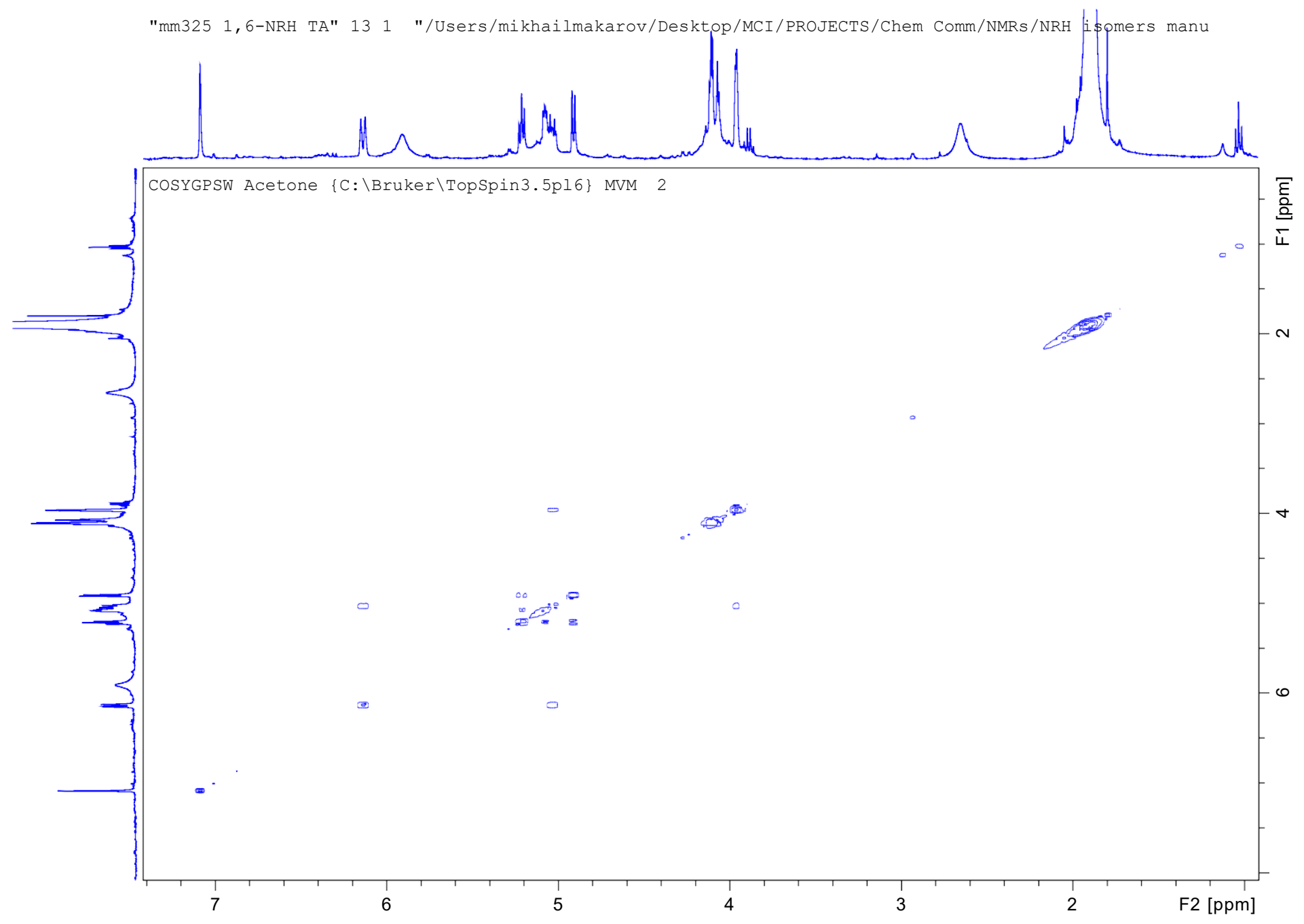

Fig. 49S ${ }^{1} \mathrm{H}-{ }^{1} \mathrm{H}$ correlation (COSY) NMR (acetone- $d_{6}$ ) of 1,6-NRH TA (9). 


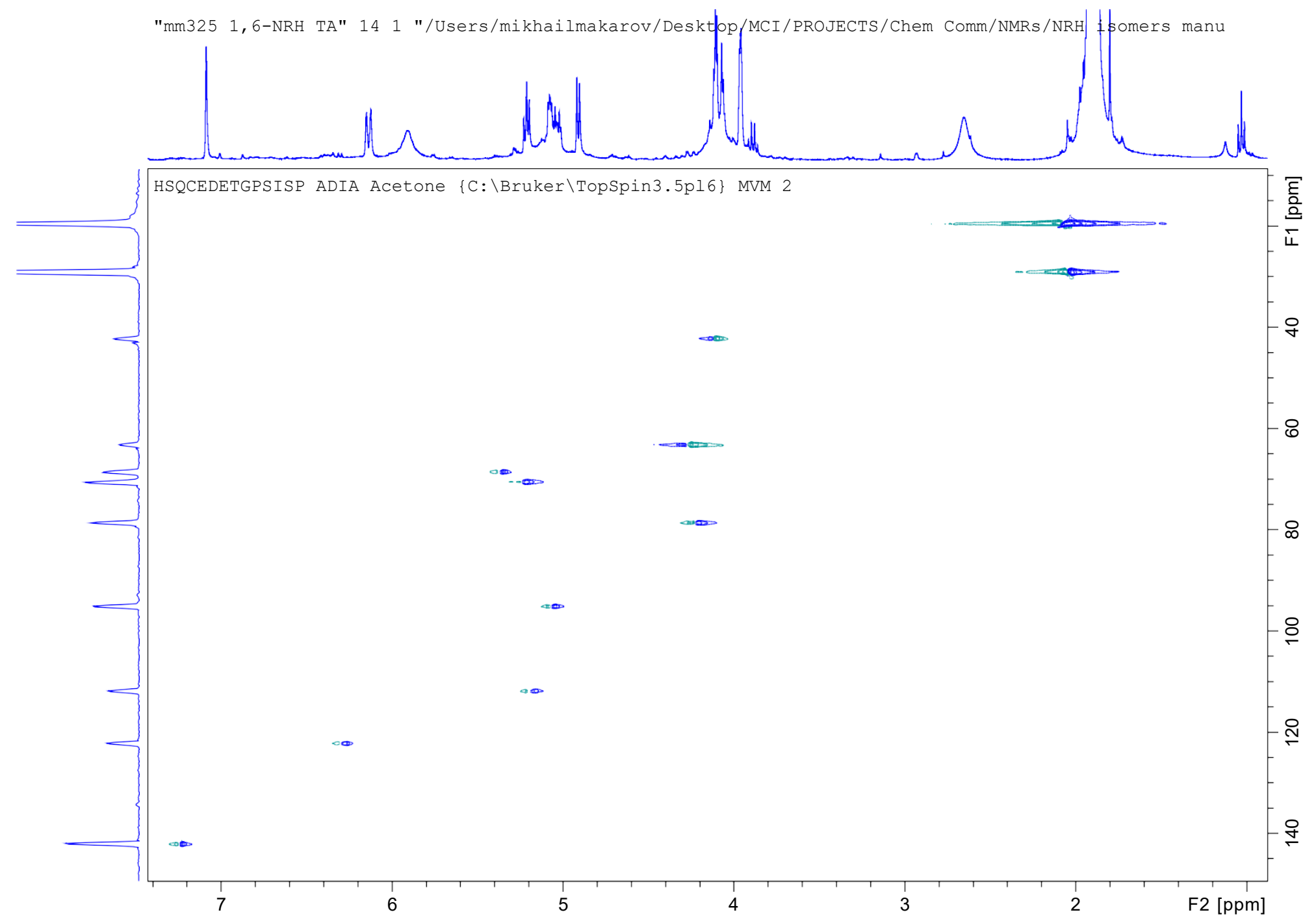

Fig. 50S ${ }^{1} \mathrm{H}-{ }^{13} \mathrm{C}$ correlation (HSQC) NMR (acetone- $d_{6}$ ) of 1,6-NRH TA (9). 


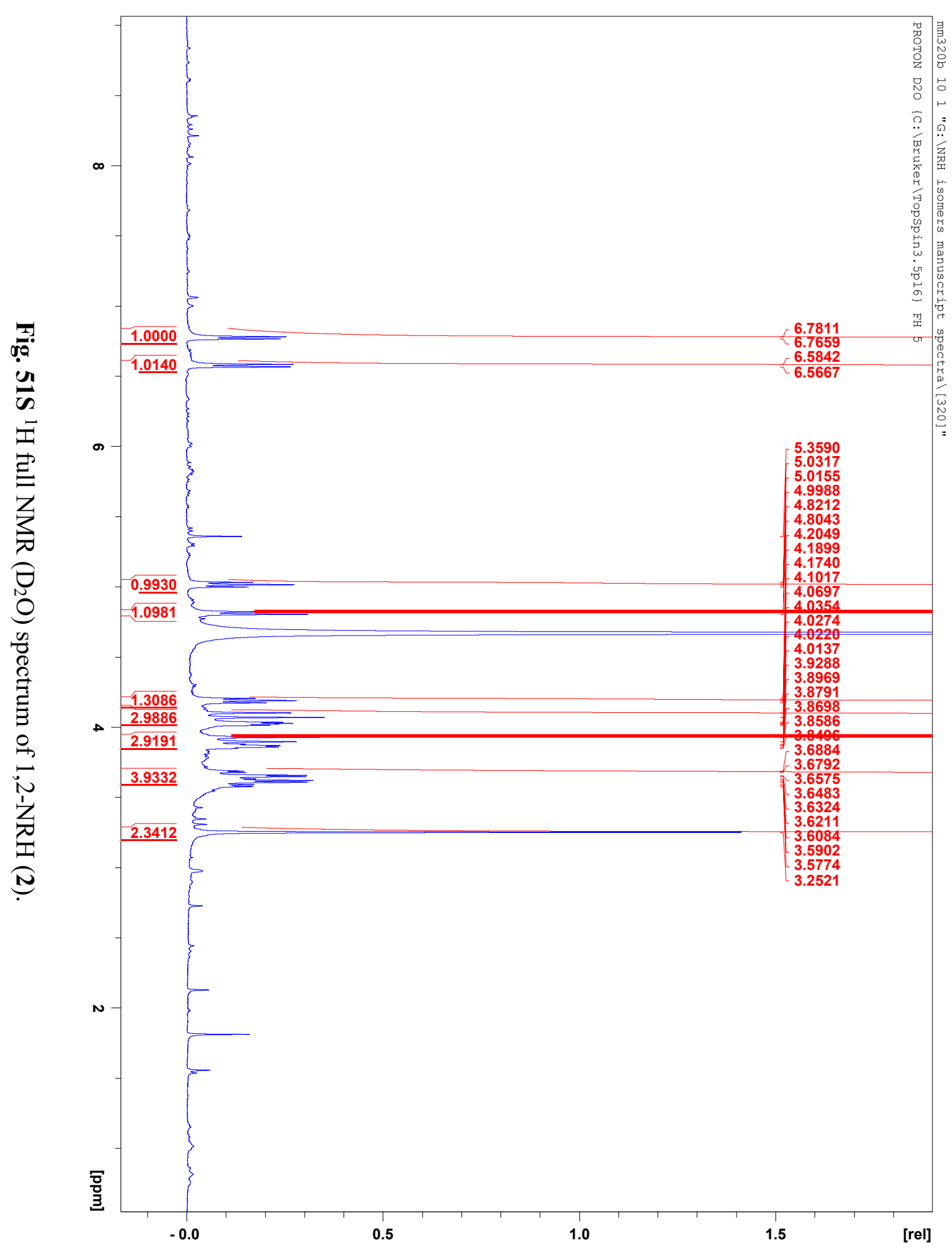




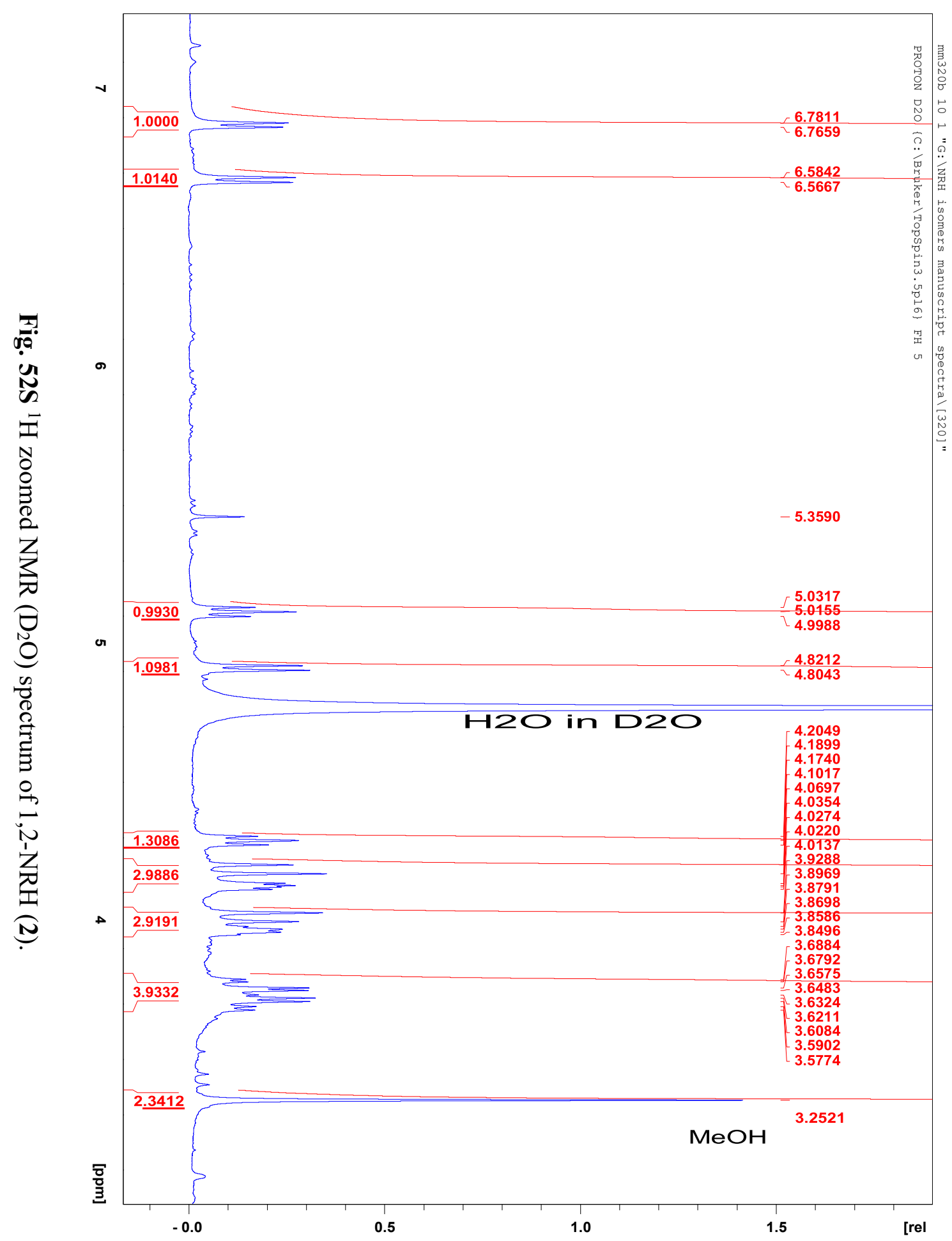




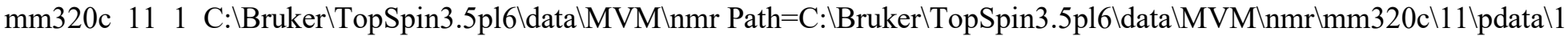

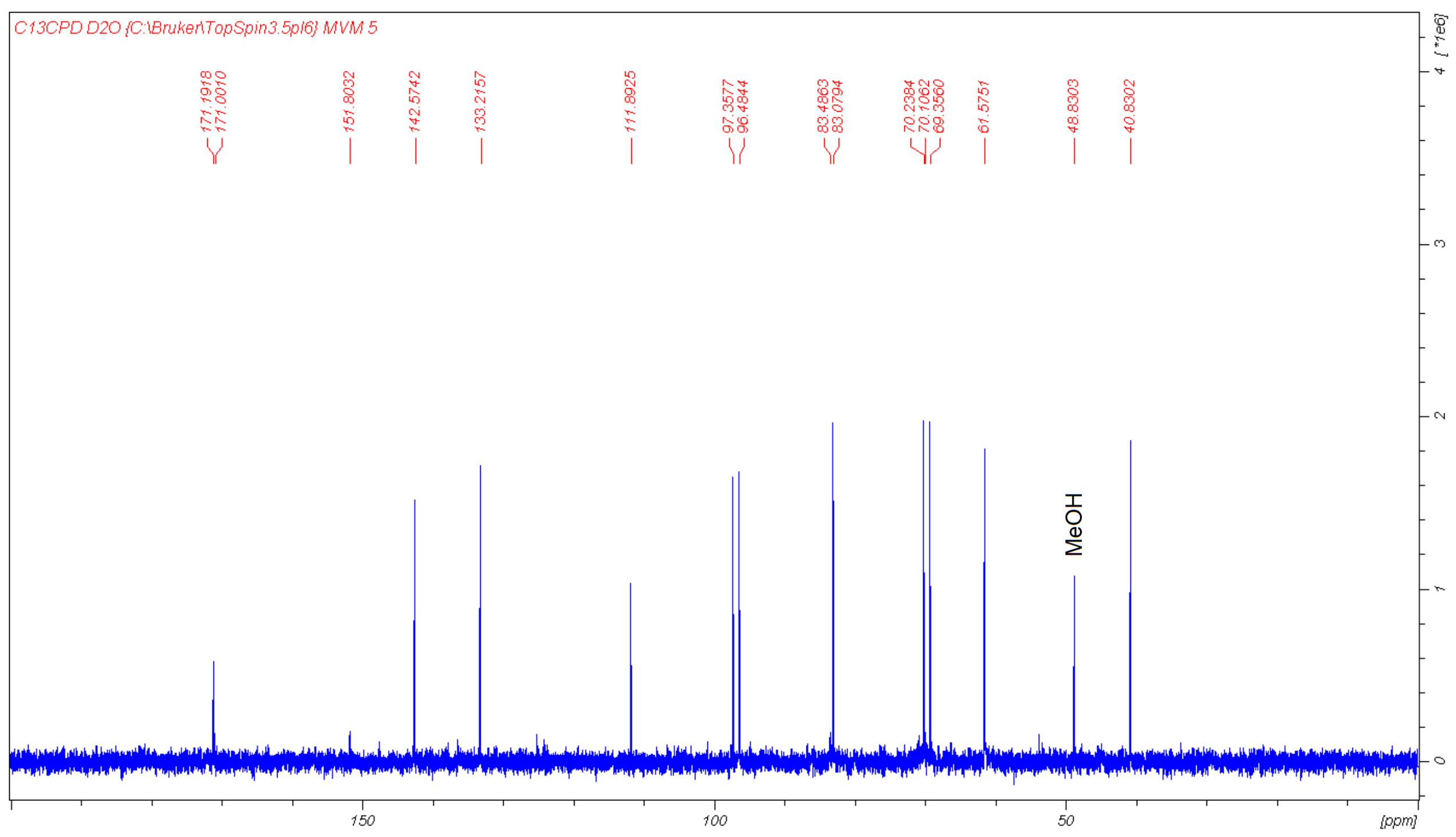

Fig. 53S ${ }^{13} \mathrm{C}$ NMR $\left(\mathrm{D}_{2} \mathrm{O}\right)$ of 1,2-NRH (2). 


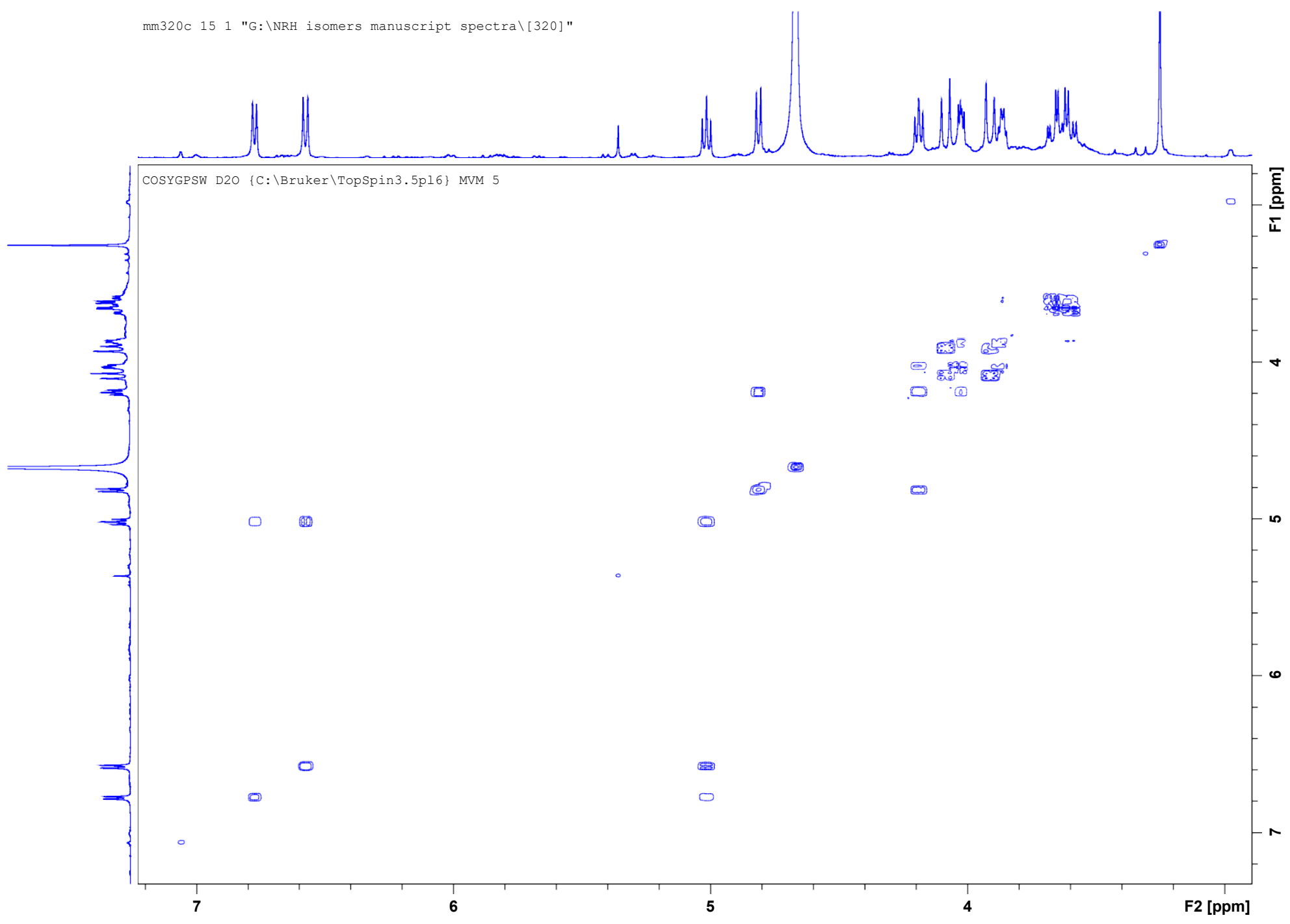

Fig. 54S ${ }^{1} \mathrm{H}-{ }^{1} \mathrm{H}$ correlation (COSY) NMR $\left(\mathrm{D}_{2} \mathrm{O}\right)$ of 1,2-NRH (2). 


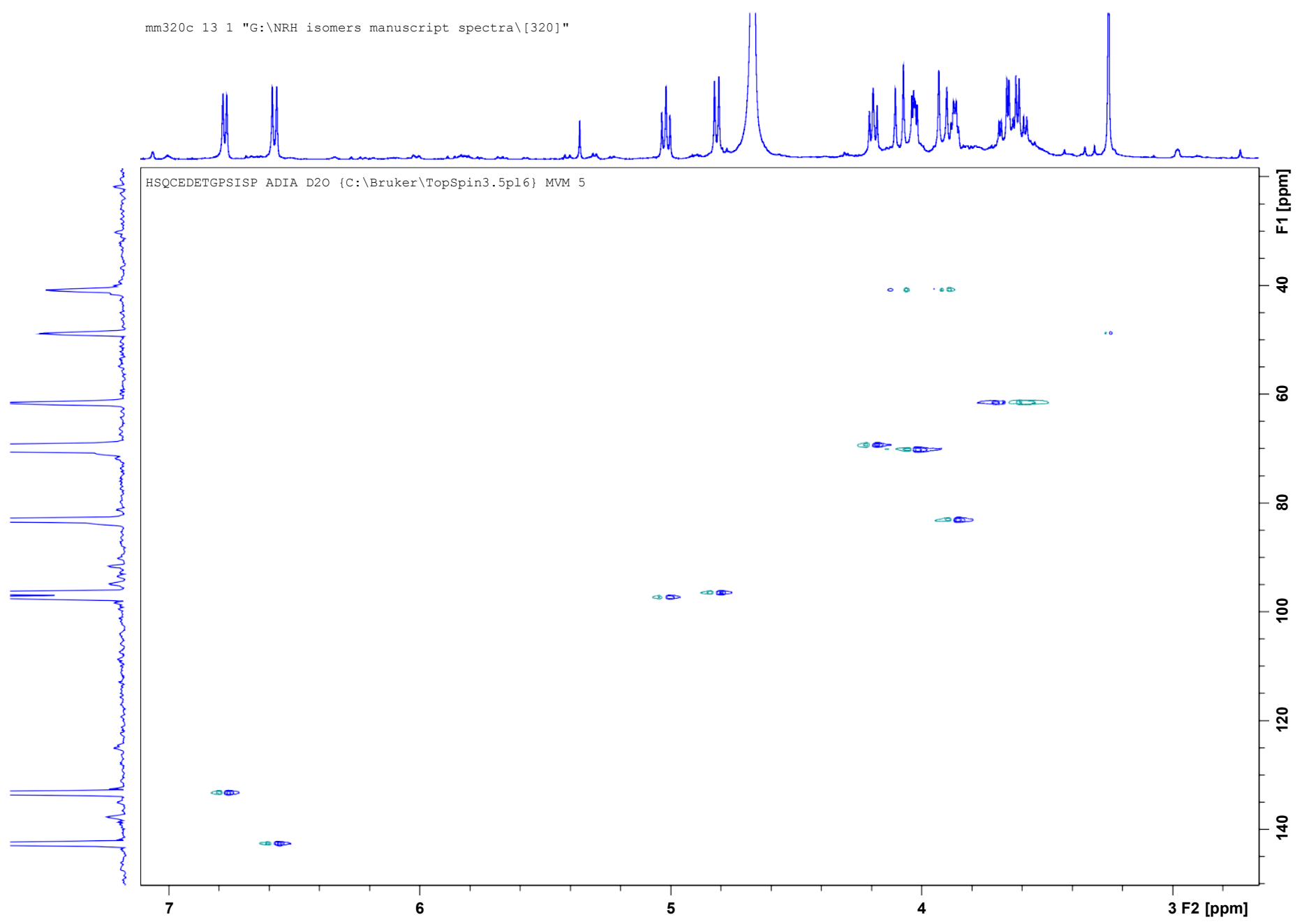

Fig. 55S ${ }^{1} \mathrm{H}_{-}{ }^{13} \mathrm{C}$ correlation (HSQC) NMR $\left(\mathrm{D}_{2} \mathrm{O}\right)$ of 1,2-NRH (2). 
MM320 - Full scan MS1

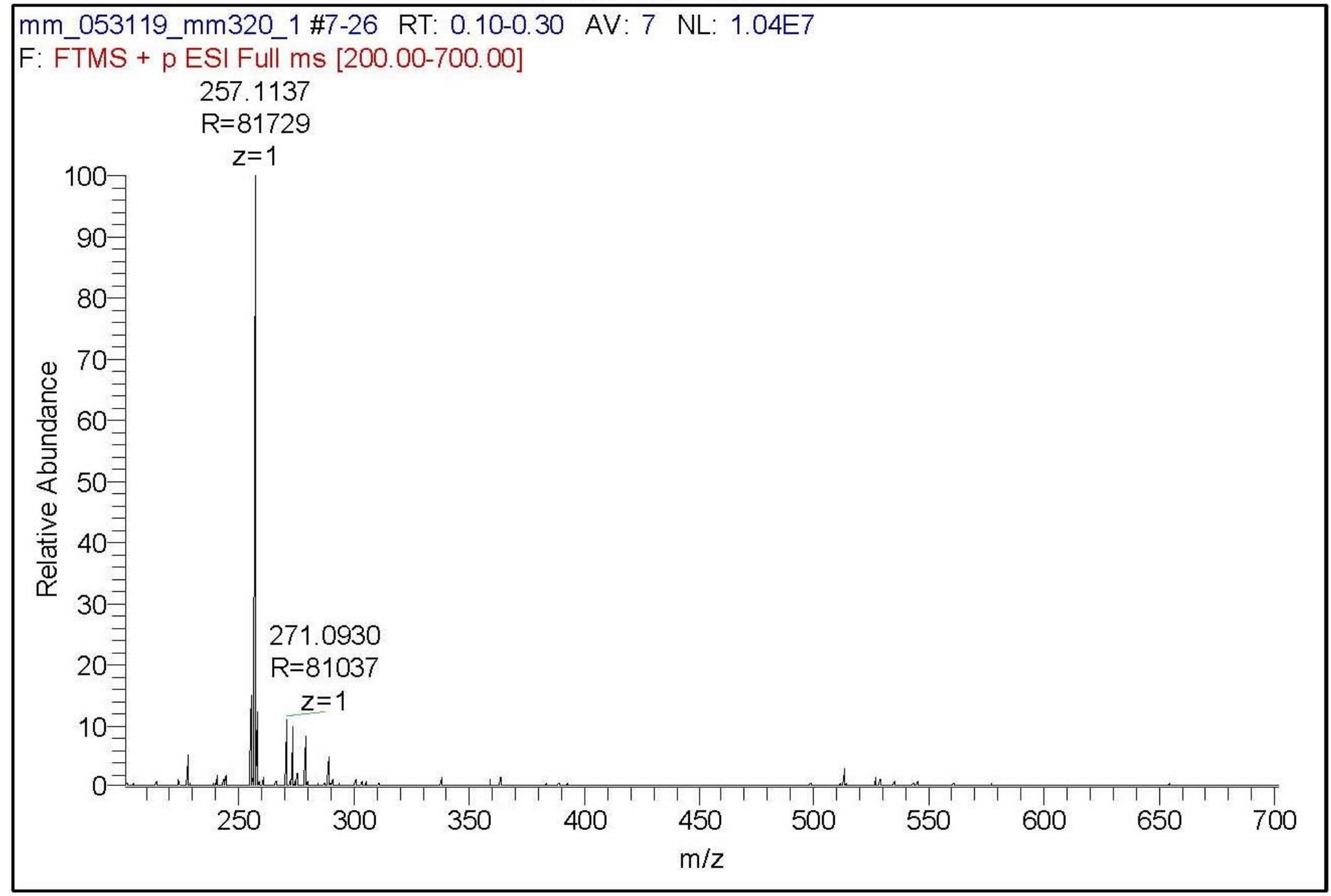

Fig. 56S HRMS (1:1 $\left.\mathrm{H}_{2} \mathrm{O} / \mathrm{ACN}\right)$ of 1,2-NRH (2): full scan. 


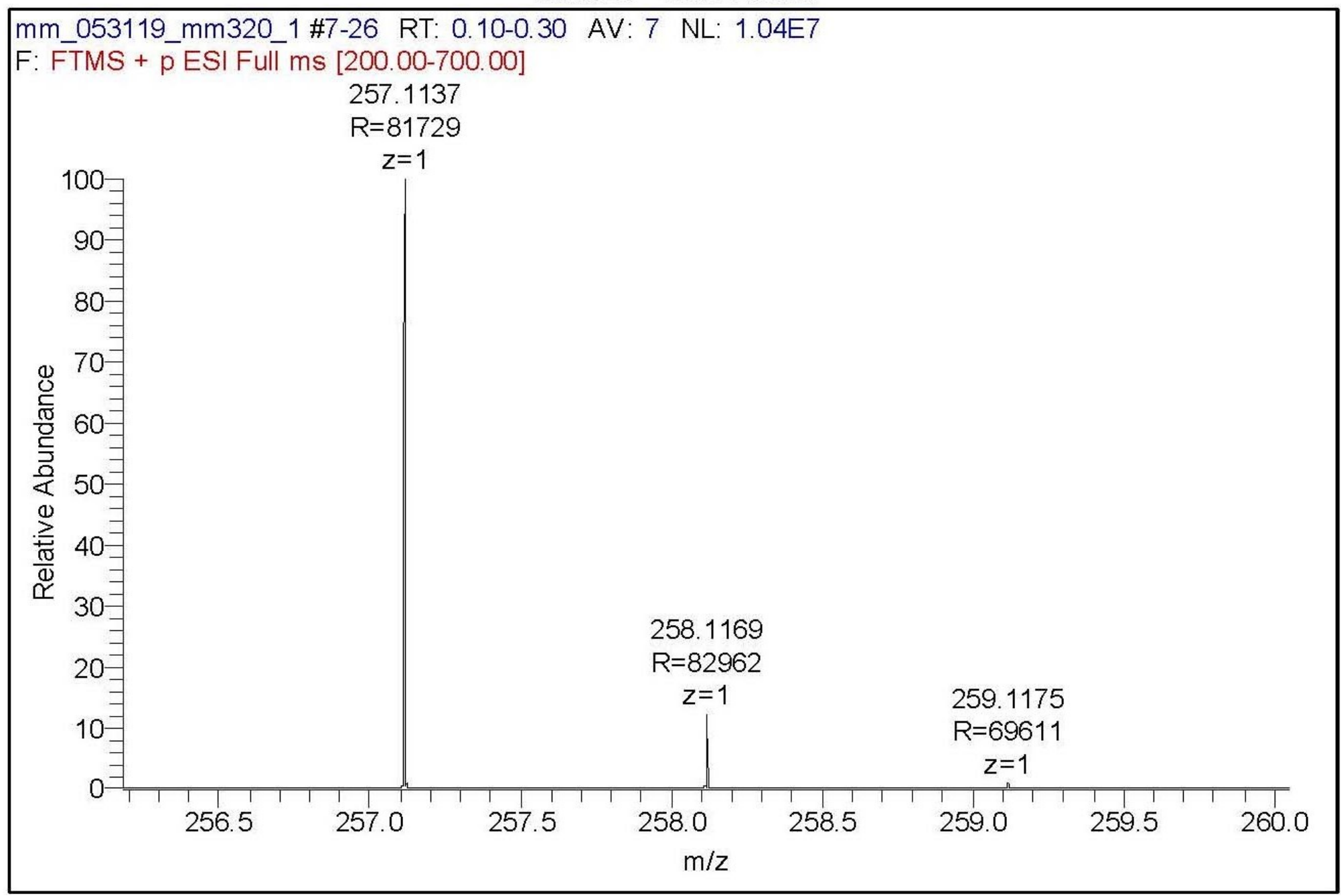

Fig. 57S HRMS (1:1 $\left.\mathrm{H}_{2} \mathrm{O} / \mathrm{ACN}\right)$ of 1,2-NRH (2): parent peak. 
MM320 - MS2 Fragmentation of 257

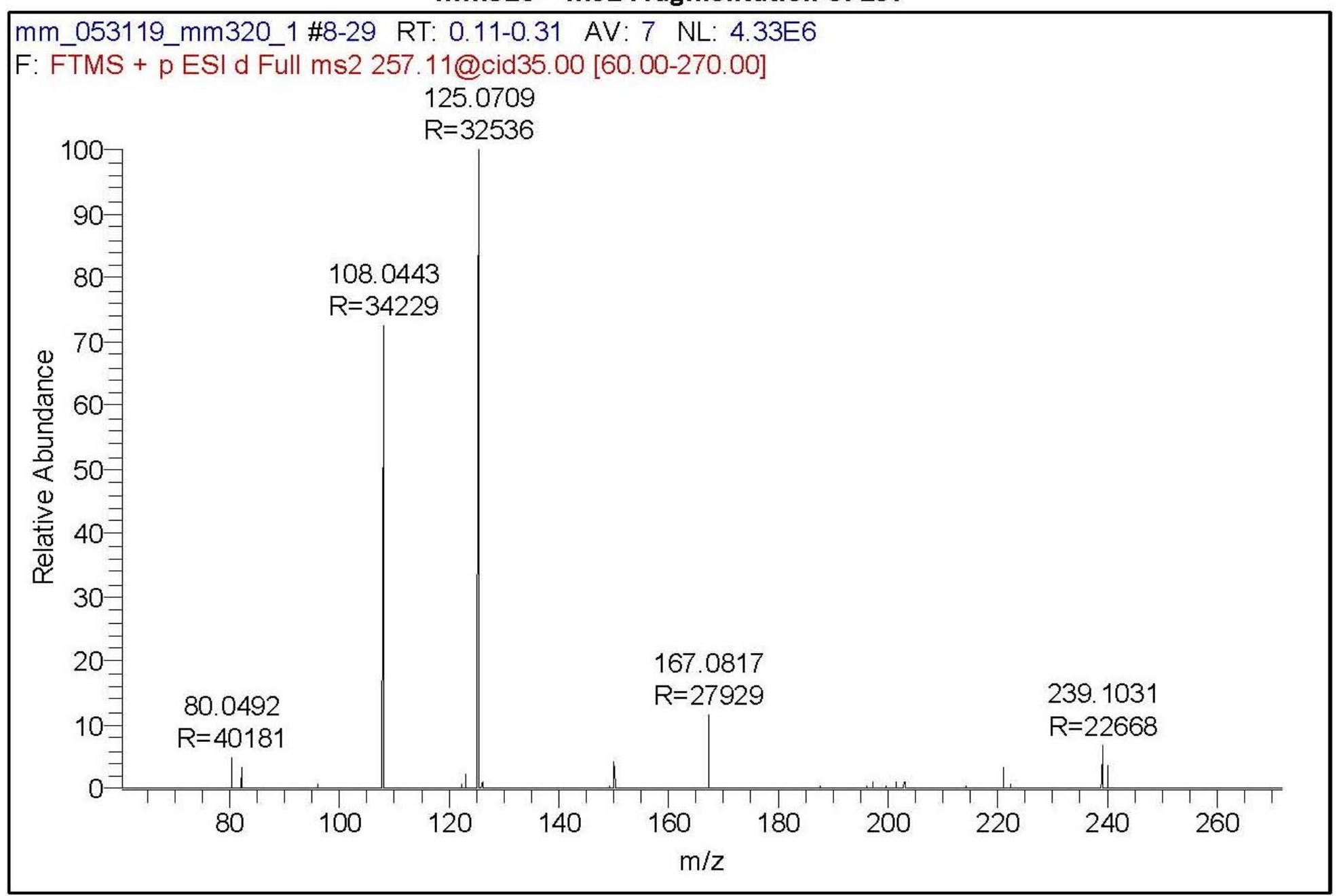

Fig. 58S HRMS $\left(1: 1 \mathrm{H}_{2} \mathrm{O} / \mathrm{ACN}\right)$ of 1,2-NRH (2): fragmentation of parent peak. 


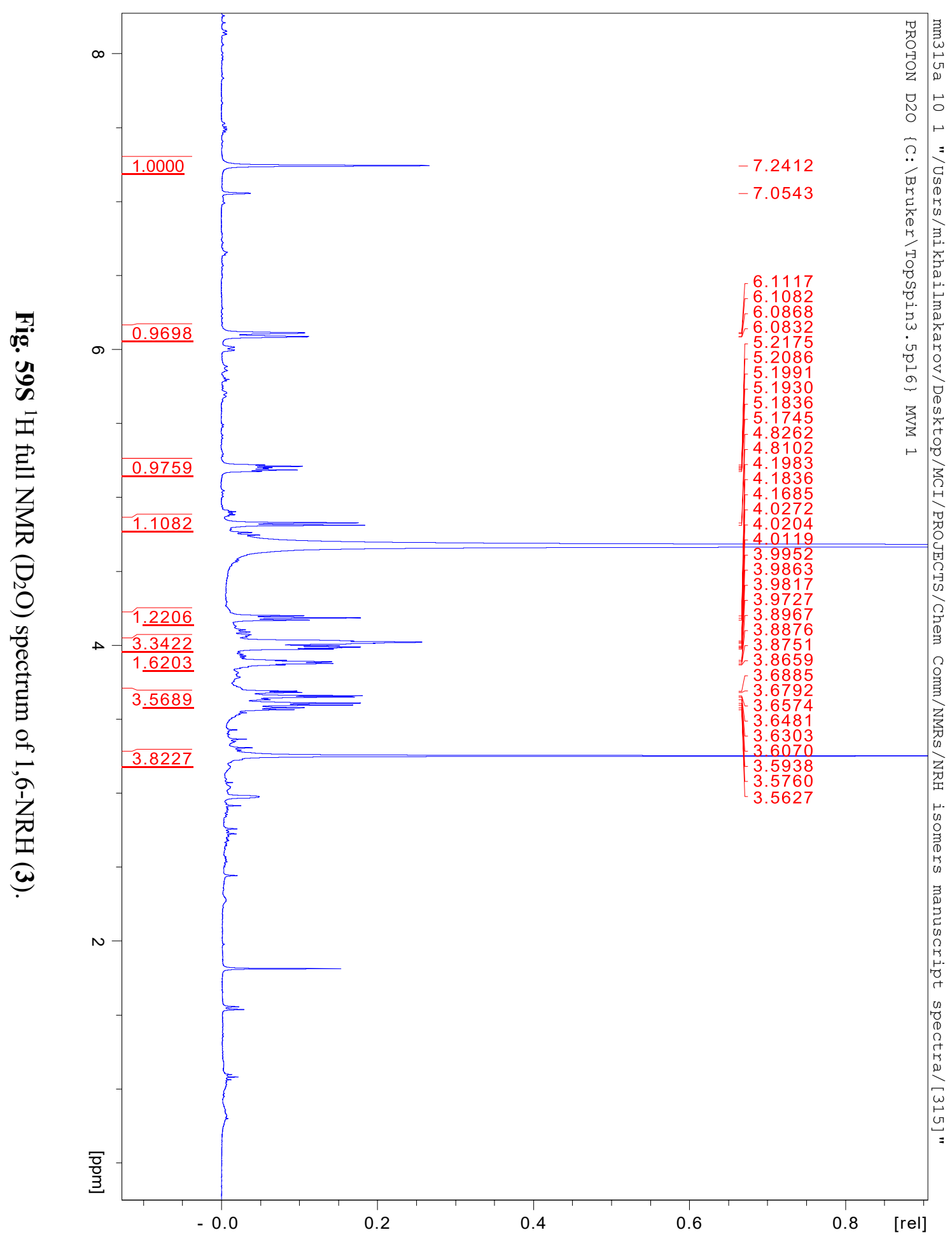


ก
.$\subseteq$
ON
I

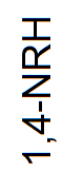

Fig. 60S ${ }^{1} \mathrm{H}$ zoomed NMR $\left(\mathrm{D}_{2} \mathrm{O}\right)$ spectrum of 1,6-NRH (3). 


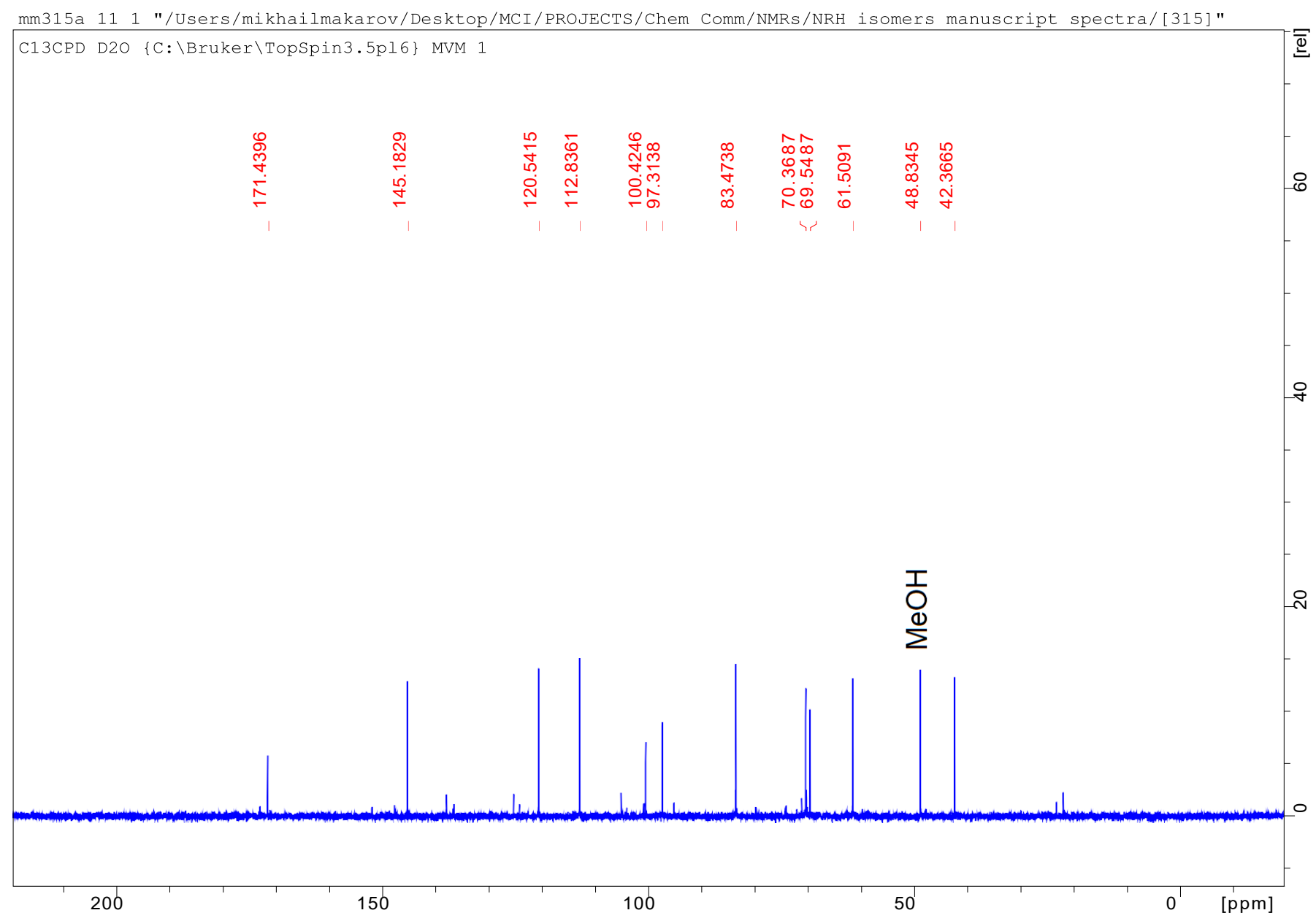

Fig. 61S ${ }^{13} \mathrm{C}$ NMR $\left(\mathrm{D}_{2} \mathrm{O}\right)$ of 1,6-NRH (3). 


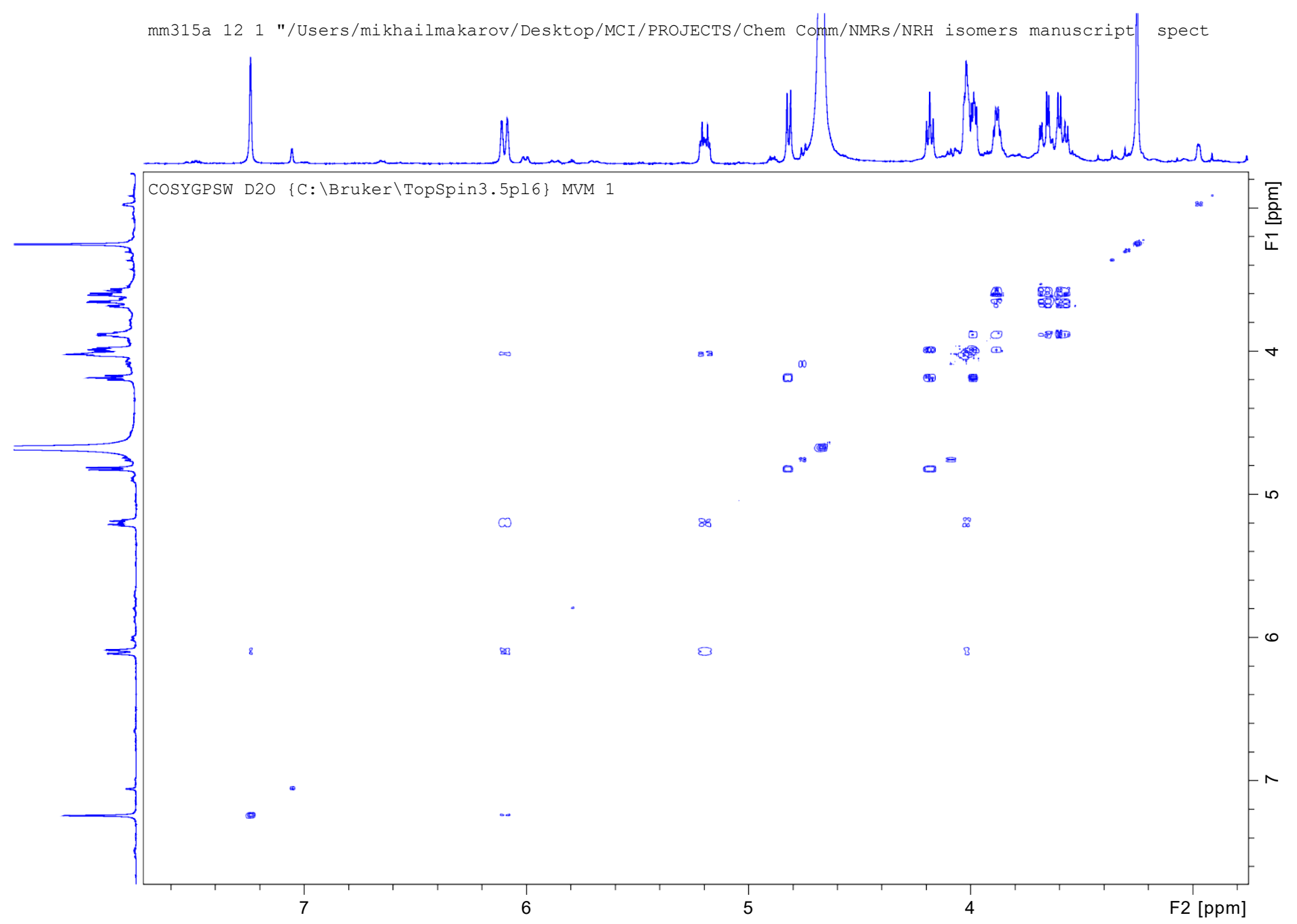

Fig. 62S ${ }^{1} \mathrm{H}-{ }^{1} \mathrm{H}$ correlation (COSY) NMR $\left(\mathrm{D}_{2} \mathrm{O}\right)$ of 1,6-NRH (3). 


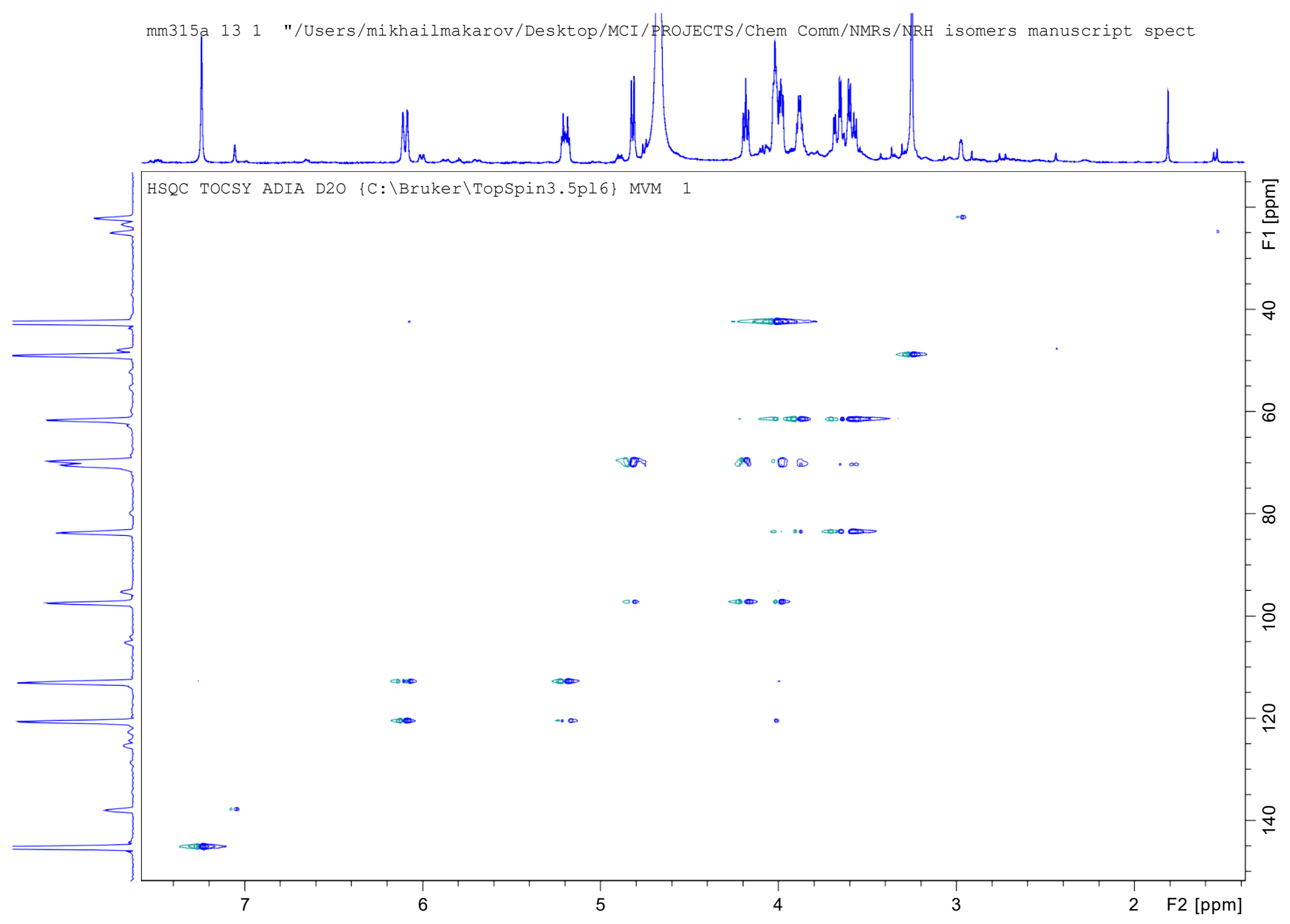

Fig. 63S ${ }^{1} \mathrm{H}-{ }^{13} \mathrm{C}$ correlation (HSQC) NMR $\left(\mathrm{D}_{2} \mathrm{O}\right)$ of 1,6-NRH (3). 


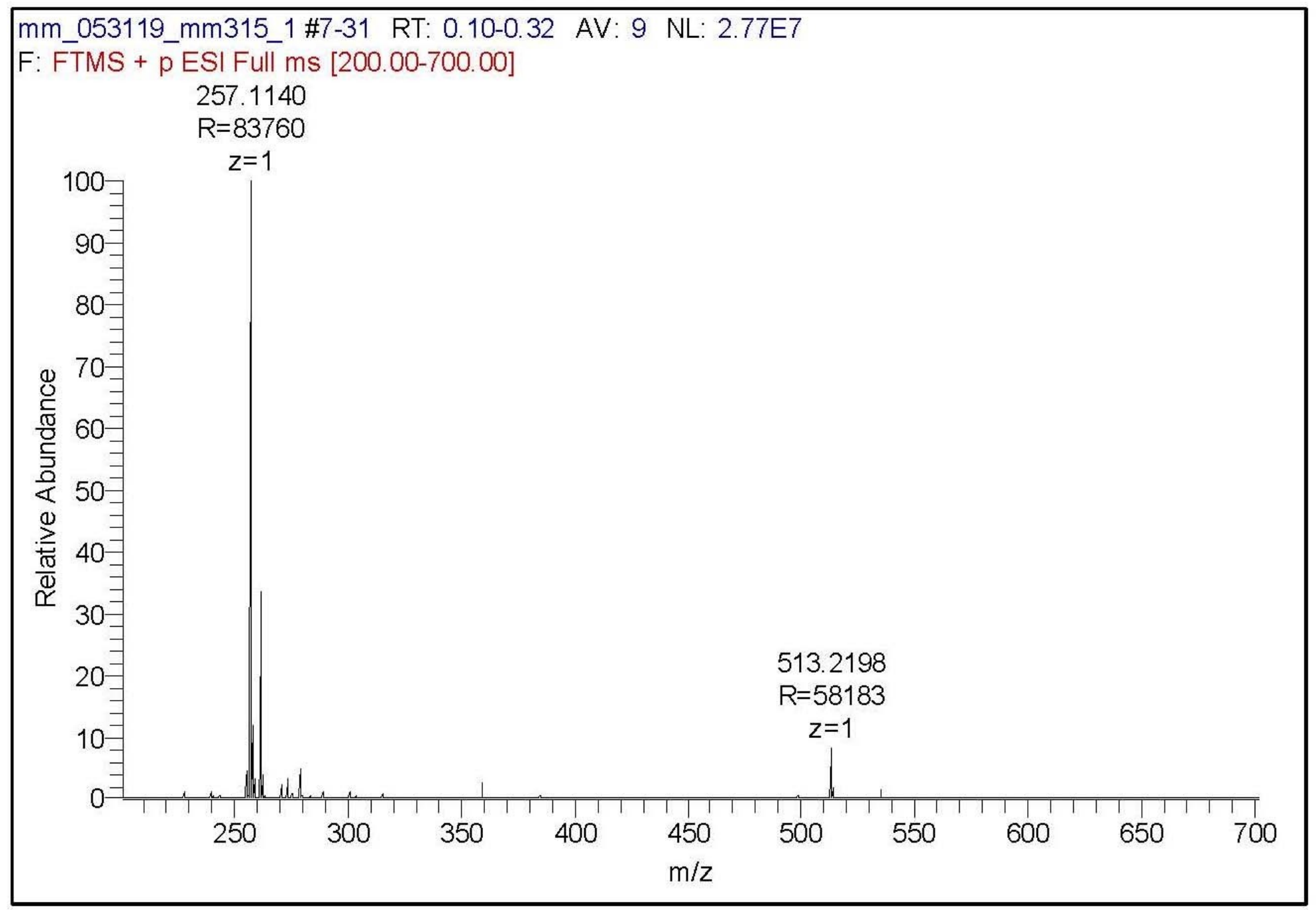

Fig. 64S HRMS (1:1 $\left.\mathrm{H}_{2} \mathrm{O} / \mathrm{ACN}\right)$ of 1,6-NRH (3): full scan. 
MM315 - MS1 Parent

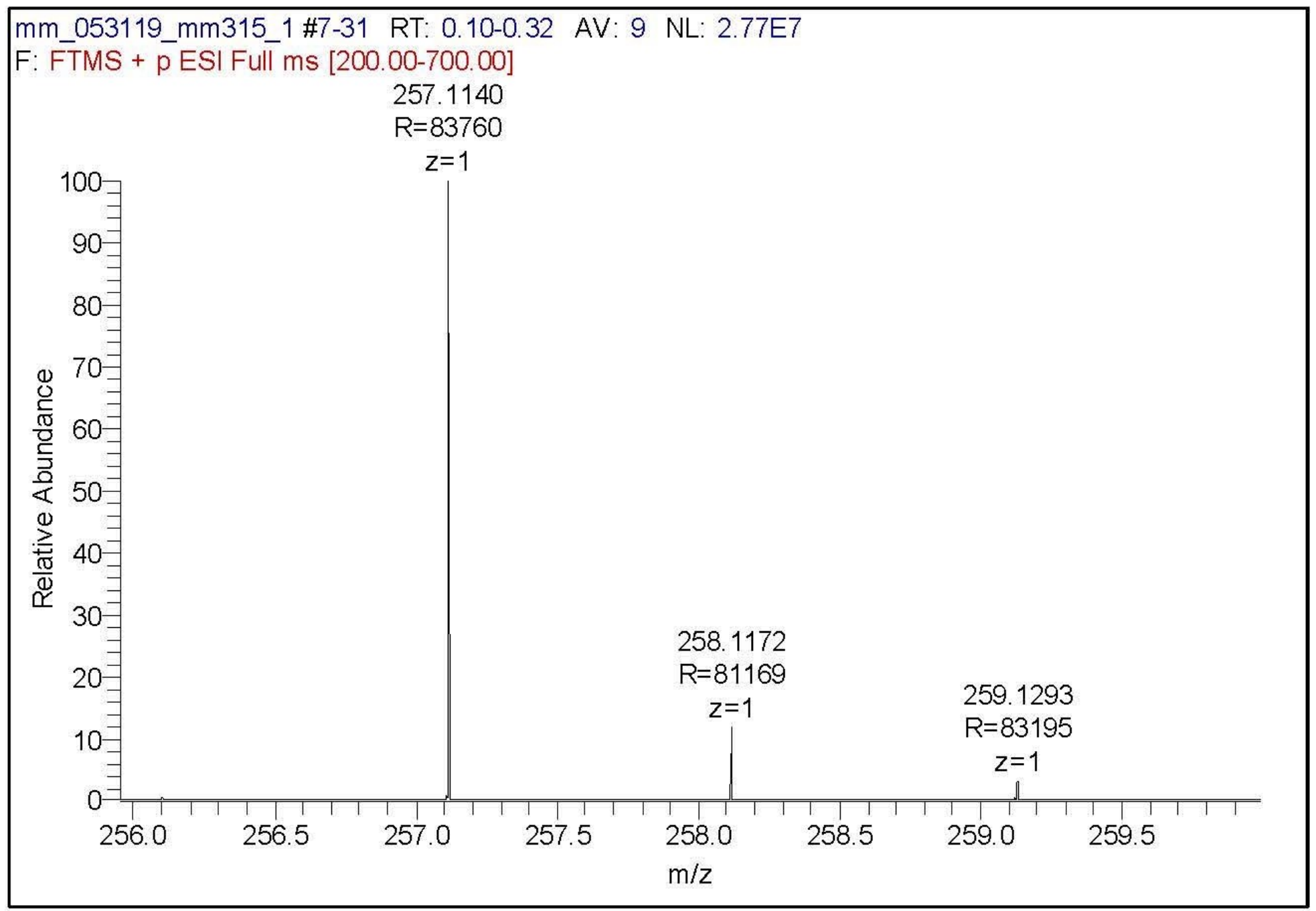

Fig. 65S HRMS (1:1 $\left.\mathrm{H}_{2} \mathrm{O} / \mathrm{ACN}\right)$ of 1,6-NRH (3): parent peak. 
MM315 - MS2 Fragmentation of 257

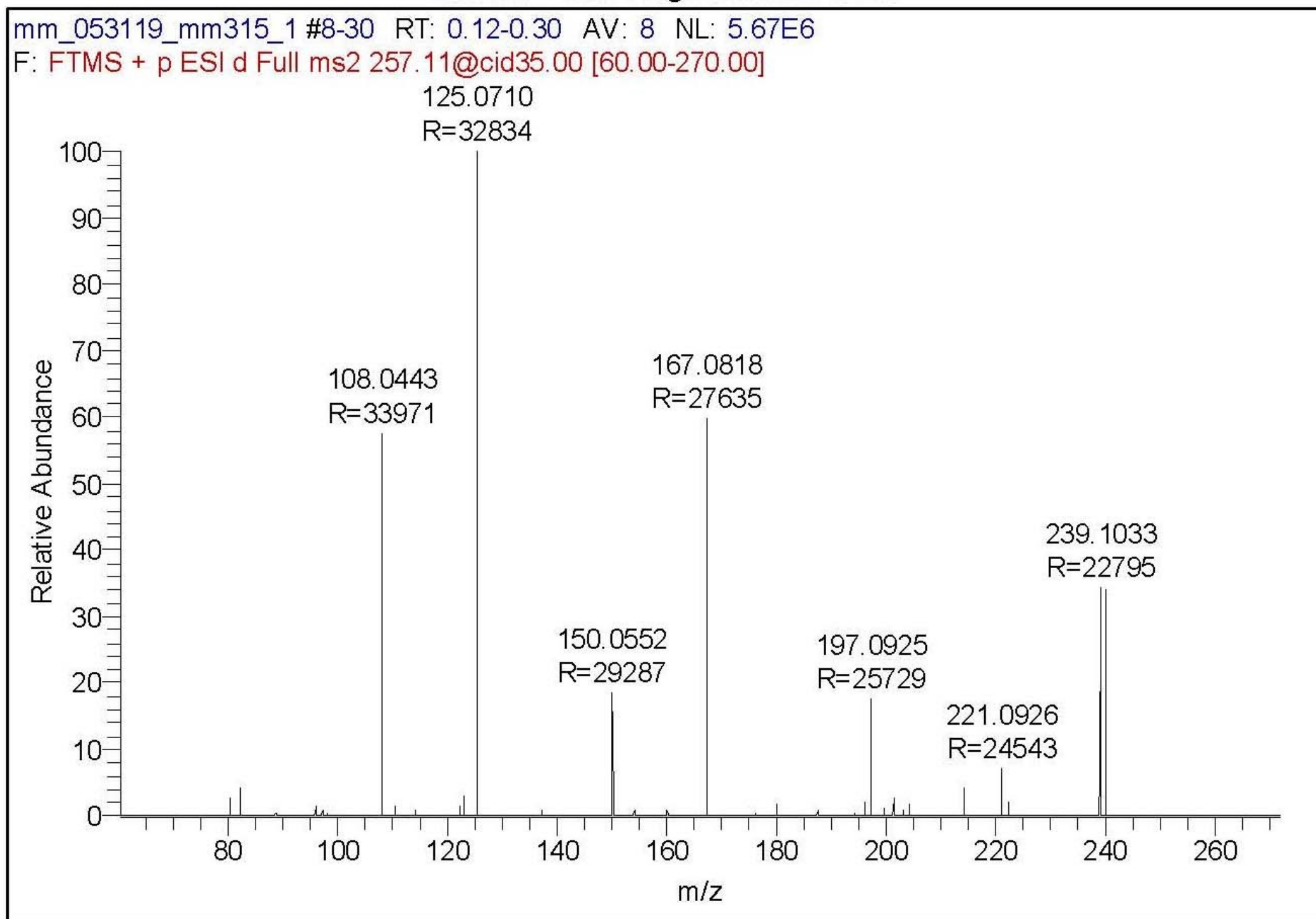

Fig. 66S HRMS (1:1 $\left.\mathrm{H}_{2} \mathrm{O} / \mathrm{ACN}\right)$ of 1,6-NRH (3): fragmentation of parent peak. 
MS2 Fragmentation Comparison of MM315 + MM320

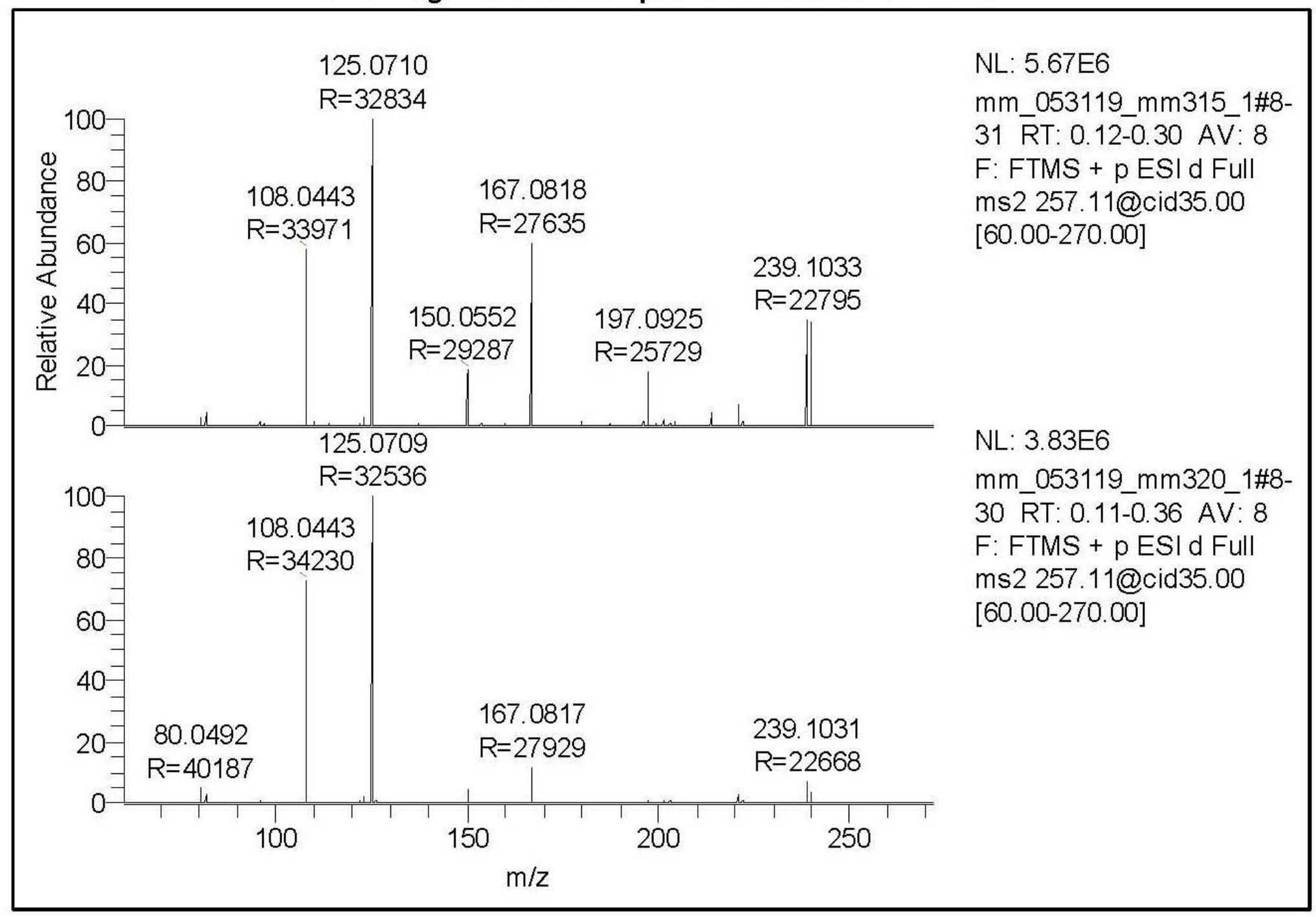

Fig. 67S Comparison of fragmentation of 1,2-NRH (2) (sample mm320) and 1,6-NRH (3) (sample mm315). 